University of Redlands

\title{
Visualizing and Analyzing Spatio-Temporal Trends in Home Values
}

\author{
A Major Individual Project submitted in partial satisfaction of the requirements \\ for the degree of Master of Science in Geographic Information Systems \\ by \\ Breck Emmerson Polk
}

Fang Ren, Ph.D., Committee Chair

Douglas Flewelling, Ph.D.

January 2020 
Visualizing and Analyzing Spatio-Temporal Trends in Home Values

Copyright $@ 2020$

by

Breck Emmerson Polk 
The report of Breck Emmerson Polk is approved.
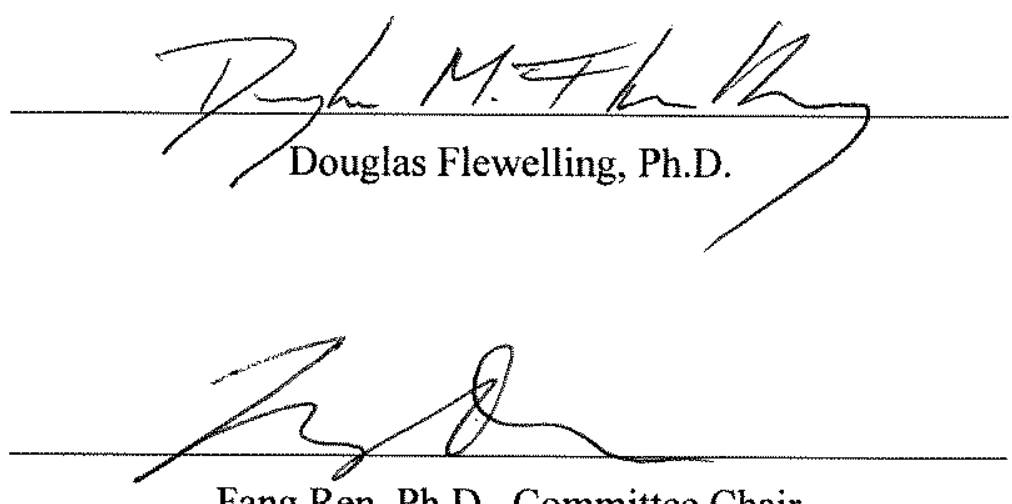

Fang Ren, Ph.D., Committee Chair

January 2020 



\section{Acknowledgements}

My completion of this project and this program would not have been possible without the assistance of so many. In particular, I would like to thank my parents and sister for their support throughout my time at the University of Redlands. During my twoyear tenure in the MSGIS program, I experienced some significant hurdles that I would not have been able to overcome without them. Their kindness and understanding have been, and will always be, sincerely appreciated. I would also like to thank my friend and fellow MSGIS alumnus, Hamish Henderson, for putting up with me throughout our classes together. I never would have imagined that having so many two person classes would have been as enjoyable as it was.

I would also like to express my gratitude to the entire MSGIS faculty. Dr. Fang Ren was an exceptional advisor, and her kindness and direction throughout the development of this project was vital to its completion. Similarly, without the continued assistance of Andrea Alvarado, I likely would have gone slightly mad trying to keep track of all of the events, workshops, classes and deadlines throughout the program. Dr.'s Doug Flewelling, Mark Kumler, and Ruijin Ma are all fantastic educators, and I consistently looked forward to their classes. Their knowledge and kindness exemplify what it is to be a stellar mentor. I am fortunate to have met many people and made many friends during my time at the University of Redlands, and they have all contributed to this project in some way or another, both large and small. 



\begin{abstract}
Visualizing and Analyzing Spatio-Temporal Trends in Home Values

by

Breck Emmerson Polk
\end{abstract}

Understanding the trends of home values through space and time can provide valuable economic and financial insights for policy makers, real estate professionals, and prospective home buyers. To better understand these trends, Johannes Moenius desired an effective and efficient method of visualizing and statistical analyzing historical home transaction data, as well as machine learning models to predict home values from assessor features. To achieve these requirements, a method of processing historical transaction point data into space-time cubes and corresponding hot spot analyses was developed, and multiple Python scripts were written to apply assessor data to Multiple Linear Regression Model and Random Forest Regression machine learning models. While the method developed for creating and analyzing space-time cubes proved effective, the machine learning models developed resulted in large errors in their home value predictions, likely due to the limited information of the assessor data used. 



\section{Table of Contents}

Chapter 1 - Introduction ................................................................................................. 1

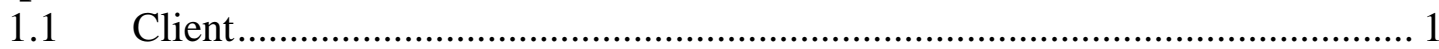

$1.2 \quad$ Problem Statement ............................................................................... 1

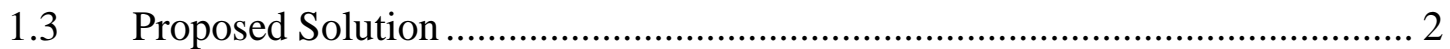

1.3.1 Goals and Objectives ............................................................................. 2

1.3.2 Scope

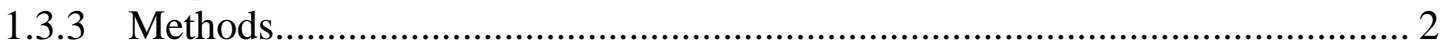

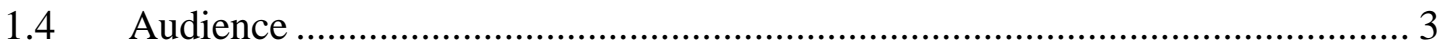

1.5 Overview of the Rest of this Report .............................................................. 3

Chapter 2 - Background and Literature Review ............................................................. 5

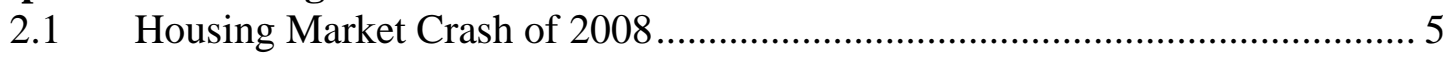

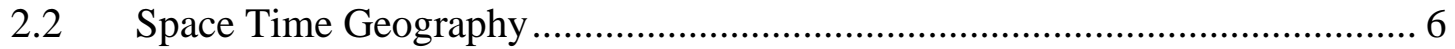

2.2.1 Visualizing Space-Time Clustering ………………..................................... 7

2.3 Predicting Home Prices............................................................................. 8

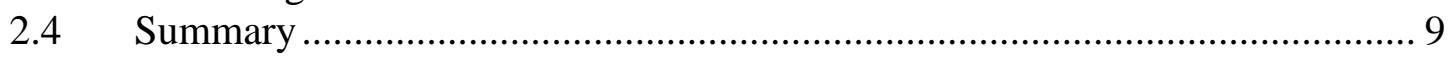

Chapter 3 - Systems Analysis and Design......................................................................... 11

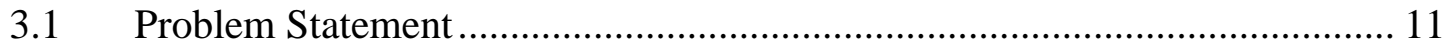

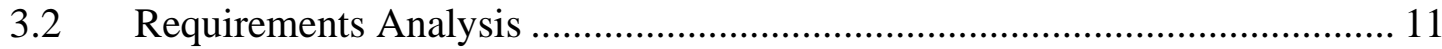

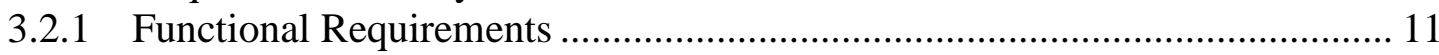

3.2.2 Non-Functional Requirements .............................................................. 12

$3.3 \quad$ System Design ..................................................................................... 13

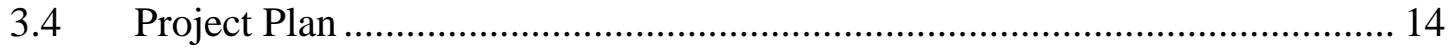

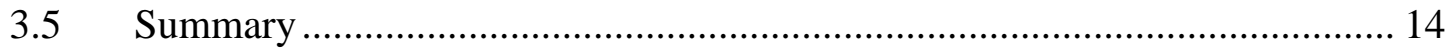

Chapter 4 - Database Design.............................................................................................. 17

4.1 Conceptual Data Model ...................................................................... 17

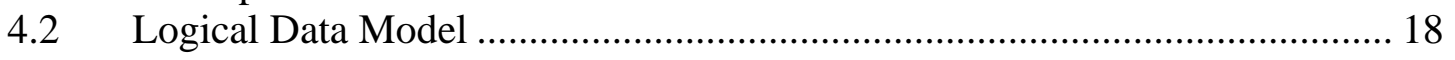

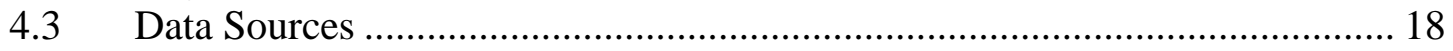

4.4 Data Scrubbing and Loading …………………...................................... 19

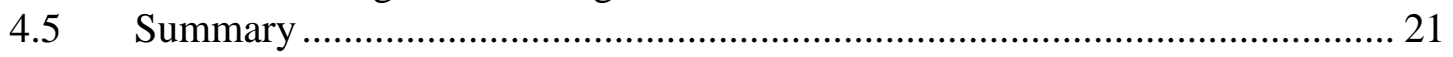

Chapter 5 - Implementation.................................................................................................. 23

$5.1 \quad$ Space-Time Cube Method ……………………………………………. 23

5.1.1 Space-Time Cube Overview …………………………………………….... 23

5.1.2 Processing Data for Space-Time Cube Implementation................................. 24

5.1.3 Implementing Space-Time Cube ………………………………………... 26

5.1.4 Visualizing and Performing Hot Spot Analysis............................................. 28

5.2 Machine Learning .................................................................................. 28

5.2.1 Pre-Processing the Assessor Data ............................................................ 28

5.2.2 Implementing the Machine Learning Models................................................ 30

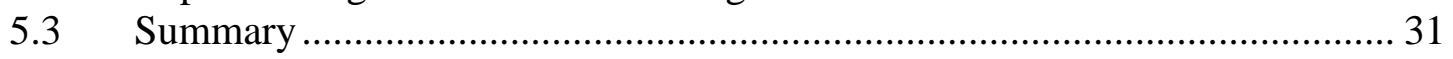

Chapter 6 - Results and Analysis....................................................................... 33

6.1 Space-Time Cube Results .......................................................................... 33 


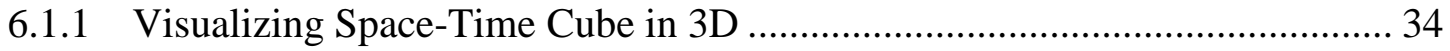

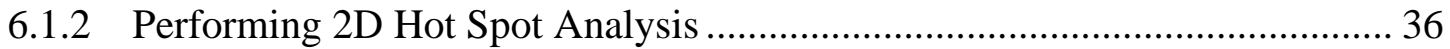

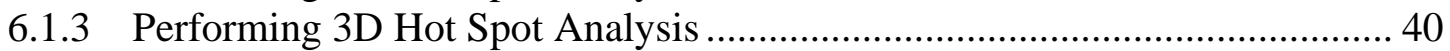

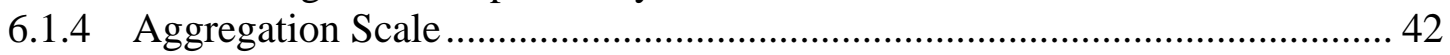

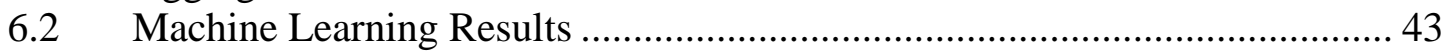

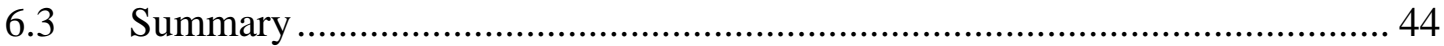

Chapter 7 - Conclusions and Future Work .................................................................... 47

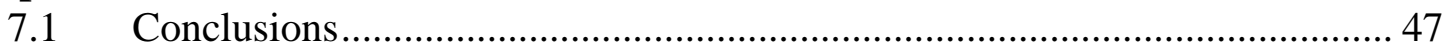

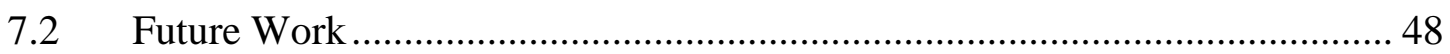

Works Cited 49

Appendix A. Scripts Written for Data Cleaning.......................................................... 51

Appendix B. Scripts written for Machine Learning............................................... 62

Appendix C. Script for Random Point Generation ................................................ 69

Appendix D. Summary Statistics for Entire Assessor Dataset .................................. 72 


\section{Table of Figures}

Figure 2-1: Still from “Inland Empire Housing Bubble” (Moenius, 2011) ................6

Figure 3-1: Space-time cube system design......................................................... 13

Figure 4-1: Diagram of the conceptual model of the historical transaction, assessor

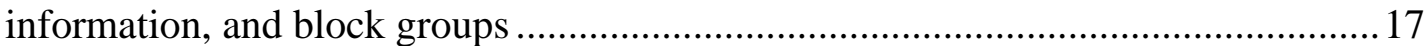

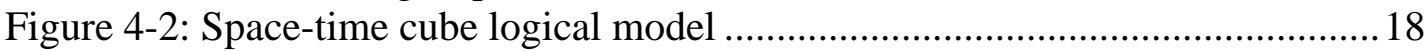

Figure 5-1: Esri diagram of a basic space-time cube (Esri b, 2019) ........................23

Figure 5-2: Space-Time Cube from Aggregated Points Illustration (Esri a, 2019) ... 24

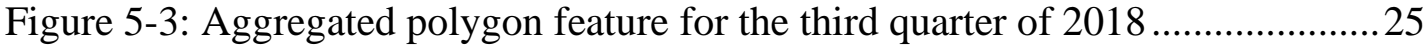

Figure 5-4: Diagram of mean transaction point generation ..................................26

Figure 5-4: Block groups for the Redlands area .....................................................2 27

Figure 5-5: Block groups for the Chino area .......................................................... 27

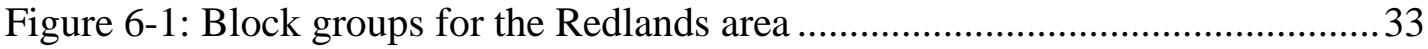

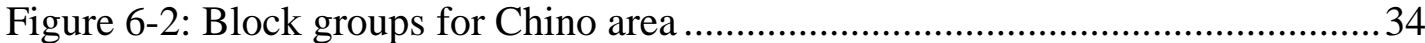

Figure 6-3: 3D Space-Time Cube of mean transaction value by block group/quarter

from Q1 2014 - Q3 2018 for the Redlands area....................................................... 35

Figure 6-4: 3D Space-Time Cube of mean transaction value by block group/quarter

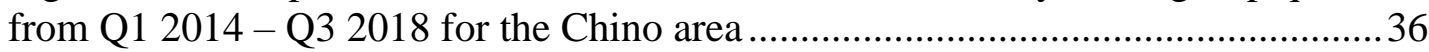

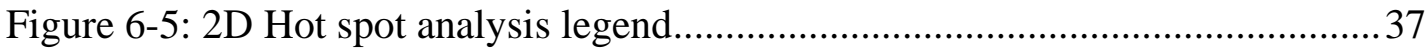

Figure 6-6: 2D Hot spot analysis of mean transaction value by block group/quarter

from Q1 2014 - Q3 2018 for the Redlands area..................................................... 38

Figure 6-7: 2D Hot spot analysis of mean transaction value by block group/quarter

from Q1 2014 - Q3 2018 for the Chino area ........................................................39

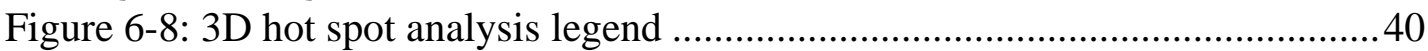

Figure 6-9: 3D Hot spot analysis of mean transaction value by block group/quarter

from Q1 2014 - Q3 2018 for the Redlands area....................................................41

Figure 6-10: 3D Hot spot analysis of mean transaction value by block group/quarter

from Q1 2014 - Q3 2018 for the Chino area ........................................................42 



\section{List of Tables}

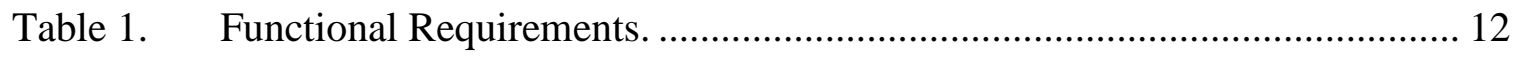

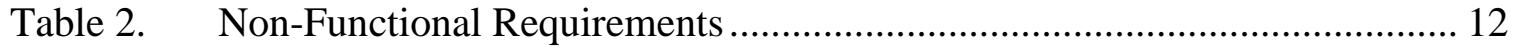

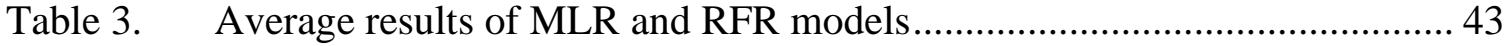

Table 4. Summary statistics of data variables ......................................................... 44 



\section{List of Acronyms and Definitions}

$\begin{array}{ll}\text { CSV } & \text { Comma-Separated Value } \\ \text { ISEA } & \text { Institute for Spatial Economic Analysis } \\ \text { MAE } & \text { Mean Absolute Error } \\ \text { MLR } & \text { Multiple Linear Regression } \\ \text { R2 } & \text { R-Squared } \\ \text { RFR } & \text { Random Forest Regression } \\ \text { RMSE } & \text { Root Mean Square Error } \\ \text { RSFR } & \text { Single Family Residence } \\ \text { SVM } & \text { Support Vector Machine }\end{array}$





\section{Chapter 1 - Introduction}

The Institute for Spatial Economic Analysis (ISEA) previously performed an analysis of the lasting effects of the 2008 Financial Recession and its influence on the housing values in Southern California. In its analysis, the institute used housing value data to determine whether there was a redistribution of wealth as a result of home value fluctuations. In other words, the ISEA wanted to determine whether or not markets of lower value homes eventually recovered and developed into markets of higher values or had the impacts of the housing market crash of 2008 persisted, thereby decimating a major store of wealth for owners. At the time of this writing in 2019, the last time the ISEA performed this analysis was in 2012, and as such, the ISEA was interested in performing a similar analysis again.

The client, Johannes Moenius, provided a dataset containing ten years of quarterly data for both assessor information and transaction histories for properties throughout Southern California. As the dataset provided was large to a point of being unwieldy, this project focused on developing a method for a smaller subsection of the data provided, the data that pertained to the county of San Bernardino, California, that the client would then be able to apply to other data going forward.

The client wished for this data to be consolidated and geocoded to allow for spatial analysis of home prices through both space and time. Fortunately, since 2012 when the ISEA last performed their analysis, Esri developed a space-time cube tool to more easily perform and visualize spatio-temporal analysis, and as such, is an ideal solution to the client's project.

Furthermore, beyond developing a space-time cube of the housing data, this project also sought to create machine learning models that would utilize the vast information provided through the dataset to predict home prices from housing assessor attributes (number of bedrooms, square footage, number of baths, etc.).

\subsection{Client}

The client for this project was Johannes Moenius, Professor and Chair of Spatial Economic Analysis and Regional Planning for the School of Business at the University of Redlands. With his educational background in Economics, Johannes has taken a particular interest in applying spatial analysis to economic problems. The client has provided the data for this project, which includes both assessor data and housing transaction data for Southern California. Beyond providing the data, the client also consulted on the economic principles implemented throughout the project.

\subsection{Problem Statement}

After the 2008 financial crisis, home values throughout the United States experienced a severe crash. Since the crash, home values have seen a strong recovery, although they have yet to reach the heights seen in the boom of the early 2000's. The client seeks a method of creating a space-time cube of home values that will allow for both the visualization and analysis of home value trends through space and time. 
Beyond the development of a means of visualizing and analyzing spatio-temporal trends in home values, this project will also seek a method of predicting home values from assessed home features.

\subsection{Proposed Solution}

One aspect of the proposed solution for this project was to develop a method of creating a space-time cube from historical real estate transaction data. To prove the efficacy of this method, example space-time cubes of the Redlands and Chino areas were to be created. The results of these example space-time cubes were to then be analyzed through hot-spot analysis, and potential explanations for the resulting trends were to be established.

A second aspect of the solution will be to utilize the assessor data provided to create machine learning models to predict home values from assessed home features. To achieve this, scripts were to be written in Python that would be trained and tested on the provided assessor data.

\subsubsection{Goals and Objectives}

The primary goal of this project is four-fold. The first and most important goal of this project is to develop a method of creating a space-time cube from historical transaction data that the client can implement in future research. The second goal is to produce example space-time cubes derived from this method. Once these examples have been created, the third goal is to analyze and provide potential explanations for the spatiotemporal trends on display. Finally, the fourth goal of this project is an aspect separate from that of the space-time cube and involves developing machine learning models that can be used to predict home values from assessor data.

\subsubsection{Scope}

The scope of this project was dependent on the number of steps involved in developing both the method for creating the space-time cube and the machine learning program, as well as on the size of the areas being studied for the example space-time cubes and the data implemented in the machine learning program.

In regard to the development and implementation of the space-time cube methodology, historical transaction data needed to be processed and cleaned for implementation and methods of implementation needed to be attempted and iterated upon until an effective workflow was established.

For the machine learning aspect of this project, again, the data provided needed to be processed and cleaned for implementation into the machine learning algorithms, and multiple machine learning method scripts needed to be written in Python, tested, and iterated upon.

\subsubsection{Methods}

Multiple methods were implemented for both the development of a space-time cube methodology and for the machine learning programs. For the development of a spacetime cube methodology, flat files containing historical transaction data needed to be 
processed and cleaned before being imported into a geodatabase and geocoded as point features. From these point features, a model needed to be developed that would translate the data in the feature into a form suitable for the creation of a space-time cube. This process involved aggregating the transaction point data, summarizing this aggregation by block group, generating random points representing the summarized monetary value, and finally generating a space-time cube by aggregating points. This method is quite involved and is discussed more thoroughly in Chapter 5. Once this method was performed, a further hot spot analysis was performed on the resulting space-time cube to provide statistical analysis of spatio-temporal trends in the data.

Regarding the machine learning programs, the data provided again needed to be processed and cleaned for use in the machine learning models. Once processed, two machine learning models were trained and tested on the data and the accuracy of the results compared. The first method was a simple multiple linear regression model with backward elimination and the second was a random forest regression model.

\subsection{Audience}

The main audience of this project was the client Johannes Moenius. However, real estate professionals and investors may find the methodologies developed in this project useful in understanding the spatio-temporal trends of home values in prospective regions of interest.

\subsection{Overview of the Rest of this Report}

The first chapter covers a general overview of the project, including discussion of the client, the problem statement, the proposed solution, the goals and objectives of the project, the scope of the project, the methods implemented, the audience of the project, and the overview of the paper.

The second chapter provides a background and literature review of the topics covered by this project. A brief background of the 2008 financial crisis and its impact on home values is provided for context, and literature relevant to spatio-temporal visualization and analysis as well as on machine learning implementations are provided for insights into the methods implemented in this project.

The third chapter outlines the system design and analysis of this project. The chapter includes a reiteration of the problem statement, discussion of the functional and nonfunctional requirements of the project, the design of the system to be implemented, and the plan outlined for the project.

Chapter four discusses the data and data structures of the project. Sections of this chapter include the conceptual and logical models of the data, sources of the data, and the processes used for scrubbing and loading the data.

The fifth chapter describes the processes involved in implementing the solutions for this project. This chapter is broken into two sections, one covering the creation and implementation of a space-time cube method for visualizing and analyzing spatiotemporal trends in home values and the other covering the creation and testing of machine learning models to predict home values from assessor data.

The sixth chapter discusses the results of the solutions implemented in the previous chapter. Again, this chapter consists of two primary sections. The first section discusses 
the results of the method and results of the space-time cube methodologies and the second discusses the results of the machine learning models.

The seventh and final chapter summarizes the completed project and suggests future work that can be done to build upon the results of this project. 


\section{Chapter 2 - Background and Literature Review}

This chapter will provide a brief background of the financial crisis of 2008 and its impacts on the housing market throughout the United States, as well as on the area of study for this project, San Bernardino County. Beyond providing this background, the chapter will also discuss methodologies of both space-time cubes and machine learning algorithms, as well as previous, similar implementations of these tools.

\subsection{Housing Market Crash of 2008}

From 2006 to 2012, the United States housing market experienced an immense correction to the preceding boom in home values in the prior decade. According to Malpezzi (2017), from 1996 to 2006, real home prices in the United States had a growth rate of approximately seven percent per annum, a stark increase from the average growth rate of just 0.4 percent per annum from 1975 to 1995 (p. 25). With such significant growth over such a short period of time, and paired with other economic factors, a catastrophic correction was inevitable. In areas with especially volatile markets, like those found in the state of California, the crash was particularly devastating.

In the California county of San Bernardino, the median home value of a singlefamily home in 2006 was $\$ 380,100$, and just five years later, the median value had dropped to $\$ 169,000$, less than half of its value in 2006 ("Median Home", 2019). With such a severe boom and bust, lower- and middle-class families, who store much of their wealth in their home's value, saw a major loss in their total net wealth. Kuhn, Schularick, and Steins (2018) estimate that from 1971 to 2007, the bottom fifty percent of the population experienced a wealth growth of ninety-seven percent, but solely from the increase in the value of their assets. In other words, any growth in total wealth for the middle and lower classes was a result of assets held, rather than a result of an increase of income or savings.

Since 2012, where the market reached its floor, home values have seen a rebound, although they have yet to reach the heights attained in 2006. Again, referring to San Bernardino County, as of July 2019 the median home value for a single-family household is $\$ 351,000$, approximately 7.6 percent less than its value of $\$ 380,100$ in 2006 ("Median Home", 2019). With this rebound in value, depending on the rate of growth, some areas may have experienced faster and stronger recoveries than others.

In addition to quantifying the changes in home values in San Bernardino, Moenius and the Institute for Spatial Economic Analysis (ISEA) (2008) visualized the change in home values from the first quarter of 2001 to the third quarter of 2008. The method they chose to visualize these changes was through $3 \mathrm{D}$ height bars in a time step animation (Figure 2-1). 


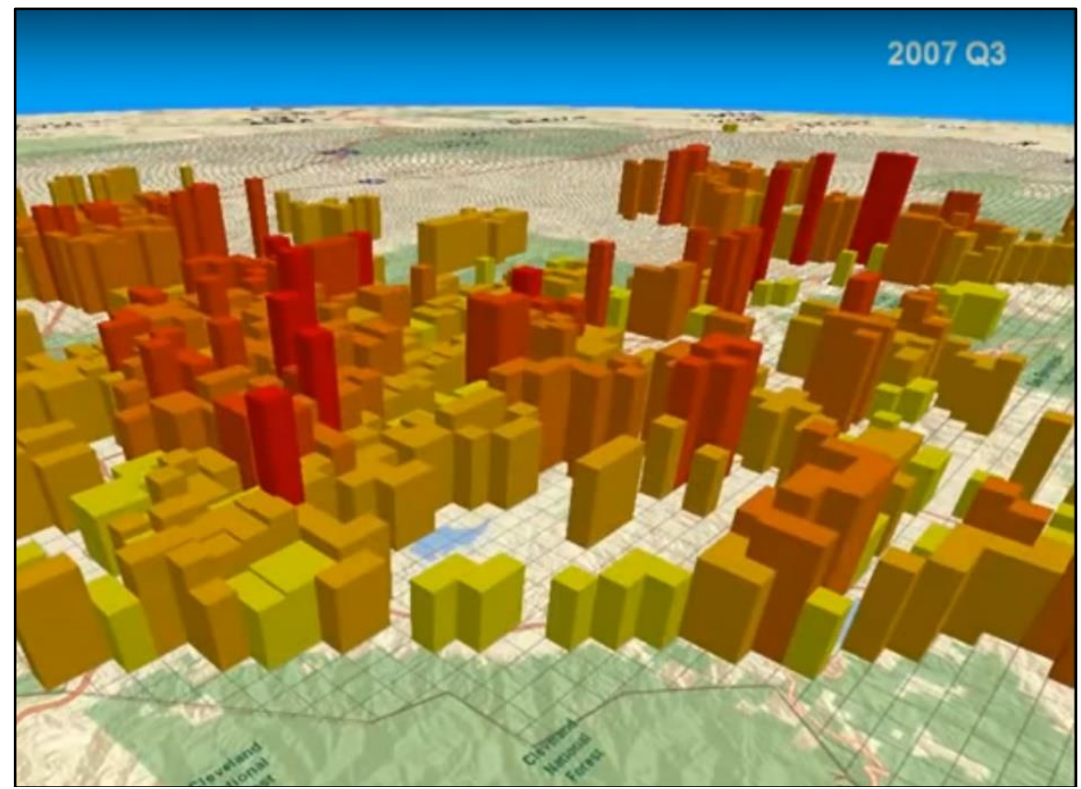

Figure 2-1: Still from “Inland Empire Housing Bubble” (Moenius, 2011)

While the method presented in Figure 2-1 does convey the necessary information regarding change in home values, a space-time cube construct specifically designed to convey spatio-temporal information from defined locations will be a more efficient and informative form of analysis and visualization.

\subsection{Space Time Geography}

A consistent observation amongst those who have studied spatio-temporal applications of GIS has been that GIS has been traditionally concerned with the presentation of static, two-dimensional maps. The first person to prominently consider the importance of time in Geography was Hagerstrand (1970) in the article "What About People in Regional Science" in which Hagerstrand dissects the information that is lost when examining people in aggregate, while ignoring the pattern of the individuals that make up that aggregate. Hagerstrand observes that when examining a region of a particular areal size, the "micro-arrangements" of the population of that region are missed, resulting in a problem for any analysis of that region (p.8). As such, Hagerstrand asserts that "nothing truly general can be said about the aggregate regularities until it has been made clear how far they remain invariant with organizational differences at the micro-level" (p. 9). To address this fact, he presents a representation of space in which the coordinates of a point in space are considered, while simultaneously representing the time coordinates of that point as well.

To visualize time coordinates of a point, Hagerstrand developed what became known as a space-time aquarium in which behaviors are presented in a prism in terms of various behaviors" "location in space, areal extension, and duration in time" (p. 11). A space-time aquarium is a rectangular prism with a vertical axis representing time intervals and the horizontal plane representing the geographic scope of the study area. This initial "halfbaked" introduction, in Hagerstrand's own words, to time Geography would have 
profound influence on how the field of GIS implemented the dimension of time into its research and analyses (p. 10).

With the further development of GIS technology, researchers such as Kraak (2003), Goodchild (2013), Kwan (2000), and Nakaya and Yano (2010) have proposed methods of further expanding upon and implementing time geography in analysis. For instance, Kwan, a prominent researcher in the area of Space-Time Geography, often cites Hagerstrand's work, as well as implements Space-Time Aquariums in the research. In Kwan (2000), Kwan develops a space-time aquarium to represent the daytime activities of three ethnic groups, African Americans, Hispanics, and Asian-Americans, in the Portland, Oregon region. In this resulting space-time aquarium, trajectories of individual space-time paths are represented by line segments connecting stationary locations. Taken as a whole, the aquarium approach can visualize human activity patterns at a disaggregate level and the density of lines in the center of the aquarium suggests a "heavy concentration of day-time activities in areas in and around downtown Portland" (p. 196).

While the space-time aquarium does a decent job of displaying clustering of the different individual space-time paths, it isn't the best method of conveying differences between individual space-time paths. To gain a better understanding of the individual paths, Kwan (2000) suggests standardizing each space-time path. To do this, the spatial coordinates of the individual space-time paths are shifted, with the home location of the individual becoming the origin $(0,0)$. In Kwan's (2008) study, she takes this process further by implementing a home-work axis, a vertical plane representing the travel from the home to work and back, into the standardized figure, thus allowing for comparing all non-home and work activities between different social groups.

Although the time-geography theory, aided by the GIS technology, provides a powerful analytical framework for exploring and visualizing spatio-temporal phenomena, understanding a large number of intertwined space-time paths may often prove challenging. To address this issue, Kwan (2000) visualizes activity-density surfaces to better reveal the overall activity patterns embedded in the individual data. This approach reduces the two-dimensional geographic space into a one-dimensional distance axis in order to represent the density in the third dimension, which would not be appropriate for the application where geographic space needs to be maintained. A recent variant of the space-time aquarium, named the space-time cube, provides an alternative to visualize spatio-temporal clustering as discussed in the following section.

\subsubsection{Visualizing Space-Time Clustering}

Compared to density surface in space and time (Kwan, 2000), a space-time cube delineates a 3D space-time space into space-time intervals (cubes) and displays the attribute of interest for each space-time cube with different saturations of hue. Space-time cubes often focus on representing statistically significant hot spots/concentrations in space and time. This method of three-dimensional visualization and analysis of spatiotemporal data has been widely used in research of crime and disease.

In their article, Nakaya and Yano (2010) implement different statistical methods of clustering to study the spatio-temporal patterns of crime in Kyoto, Japan. As the two note, depending on the temporal patterns of hot spots, different strategies of addressing crime may be advisable. In instances where crime clusters occur in short time intervals or are cyclical, investing in access to rapid police response is recommended, while when 
crime clustering is persistent through time, it would be advisable to invest in more permanent efforts to change the social or environmental conditions in the area (Nakaya and Yano 2010, p. 224). Whereas previous methods of tracking the changes in crime hot spots involved statistical testing of a few time intervals as well as of specific areas in the region of study time, often intervals before and after some change in the environment of the region of study, a three-dimensional space-time cube allows researchers to present crime events visually and "simultaneously comprehend the temporal duration as well as the spatial extent of different crime clusters" throughout the entire spatio-temporal study area (2010, p. 225).

Another implementation of space-time cubes for visualizing and analyzing clustering by Kang, Cho, and Son (2018), allowed researchers to study traffic accidents involving the elderly in Seoul, South Korea. By first generating a space-time cube, then performing emerging hot spot analysis and space-time kernel density estimation, the team was able to reach three conclusions regarding the spatio-temporal traffic accident data. First, from hourly time intervals, the team concluded that from the morning to the afternoon, hot spots were concentrated in areas North of the Han river, while from daytime to evening, hot spots were widely dispersed. Second, monthly time intervals indicated that hot spots were weak in the harsher seasons of winter and summer, while hot spots were strong in recreational areas during the temperate seasons of spring and fall. Finally, when considering the entire period of the year studied, 2013, hot spots of traffic accidents involving the elderly were increasing in areas North of the Han River.

Space-time cubes provide a valuable means of not only visually representing spatiotemporal hot spots/clustering, but also of analyzing the statistical significance of those hot spots/clusters. Vital insights can be made from the trends observed from space-time cubes, insights that are unachievable by traditional GIS methods unconcerned with the added dimension of time.

\subsection{Predicting Home Prices}

Machine learning is a rapidly growing area of research in the field of data science, and the algorithms produced are being utilized in myriad use cases. For instance, with the advent of machine learning algorithms built for prediction, researchers have created methods of predicting spread of disease, rates of violent crime, and pertinent to this paper, the values of real estate. In their research, McClendon and Meghanathan (2015) implement and compare the results of three machine learning models (linear regression, additive regression, and decision stump) in predicting rates of violent crimes from community and law enforcement predictive features. From their results, the two conclude that of the three methods, the linear regression model was the most accurate, with the highest correlation coefficient and the lowest resulting errors. When applied to data from Mississippi, the model predicted the mean counts of murder and rape per one hundred thousand people to be 6 and 36, compared to the actual counts of 7 and 31. While not perfectly accurate, the model produced relatively close predicted rates of violent crime, and as such, could be applied to other cities to help law enforcement determine where potential violent areas may develop.

Historically, models for predicting home values have relied on hedonic models, or models that assume "goods are valued for their utility-bearing attributes or characteristics" (Rosen, 1974, p. 34). Put more simply, models of predicting home prices 
traditionally rely on characteristics of the home like the square footage of the home or the number of bedrooms and bathrooms in the home (Kaboudan \& Sarkar, 2007, p. 24).

Before machine learning rose to prominence, these hedonic models were applied in stochastic processes. Whereas stochastic statistical methods aim to establish a model for relationships between variables and the significance of those relationships, while also allowing for predictions, machine learning algorithms are concerned only with making predictions and getting the most accurate results (Stewart, 2019). Essentially, a stochastic model is meant to be able to be interpretable, so that the methods of the model can be critiqued, while a machine learning model will be critiqued by the data inputted and the results generated.

Baldominos and others (2018) implement and compare four different machine learning models, Support Vector Machine (SVM), K-Nearest-Neighbors, Ensembles of Regression Trees, and Multi-layer Perceptron, in predicting home values in the Salamanca district of Barcelona, Spain from three categories of variables (locational, home characteristics, and ad characteristics) (p. 7). In their research, Baldominos and otehrs first perform exploratory analysis of the data they will be using to train their machine learning algorithms. These analyses resulted in Pearson Correlation Coefficient ratings, a correlation matrix, and a boxplot of the price distributions based on locations of real estate (pp. 8-10). From these, it was determined that variables of property size held the strongest influence over price and that location was also an important variable in predicting price. Once the most relevant variables were identified, the team then implemented the four machine learning techniques on both normalized and nonnormalized data. Their final results determined that the method of Ensemble Regression Trees performed the best in regard to both the mean absolute error and the median average error.

The advent of machine learning, and computers powerful enough to perform the complex algorithms inherent to it, has allowed for researchers, policy makers, and businesses to more accurately predict outcomes from the vast amounts of data currently being gathered.

\subsection{Summary}

With the client's previous work relying on time-step animations to convey trends in housing data through time and space, a Space-Time Geography approach like that of a space-time cube will provide a more efficient and informative method of not only visually conveying spatio-temporal trends, but of quantitatively analyzing it as well. By implementing a space-time cube solution, this project will provide the client a means of inspecting spatio-temporal clustering throughout the region of study, a result not easily achieved with the previous method of analysis.

Beyond visualizing and analyzing clustering in the data, machine learning models will provide the client with further insights into the housing market of Southern California. This project will produce machine learning models to predict a home's value from characteristics of the property and of the surrounding area. To build these models, the data will need to be analyzed and processed, and the results of each model analyzed for their accuracy. 



\section{Chapter 3 - Systems Analysis and Design}

To ensure a project is completed efficiently and to the standards outlined by the client, it is vital to establish a thorough and viable project plan. To that effect, this chapter will elaborate on this project's plan and design. Section 3.1 will reiterate the problem posed by the client. Section 3.2 will list the requirements of the project as established by the client and advisor of the project. Section 3.3 will discuss the system design of the project. Finally, section 3.4 will establish the original project plan and how it changed throughout its lifecycle.

\subsection{Problem Statement}

As a result of the 2008 financial crisis, home values throughout the United States experienced a severe correction to the preceding decade's boom in home prices. While there has been a recovery in home values since 2008, the client would like to be able to analyze the rates of recovery in different geographic areas. Previously, the client implemented a method of representing home values through time and space that relied on a time step animation of 3D height bars. While this method worked to visualize the data, it does not allow for easy analysis or visual comparison between time intervals. To address these shortcomings, a space-time cube of home values will allow for statistical analysis of spatio-temporal trends throughout the data, as well as for all time intervals to be visually compared simultaneously. Beyond the development of a space-time cube, a secondary deliverable of a machine-learning algorithm will be created that will allow the user to predict home values from homes' assessed features.

\subsection{Requirements Analysis}

A requirements analysis typically consists of determining the functional and nonfunctional requirements of a project. Functional requirements establish what the endproduct should produce, while non-functional requirements establish constraints within which the end-results should operate.

\subsubsection{Functional Requirements}

As this project is concerned with developing a method of producing and analyzing a space-time cube, as well as with developing a machine learning algorithm to predict home values, there are multiple functional requirements that must be met (Table 1). The most important requirement for this project is the development of a method to produce a visualization of spatio-temporal data in the form of a space-time cube. A second aspect of this requirement is establishing methods of analysis for the resulting space-time cube. These methods will allow the client to produce similar space-time cubes and analyses for future research. Finally, to prove the efficacy of these methods, example space-time cubes and analysis of them needed to be created. 
Table 1. Functional Requirements.

\begin{tabular}{|l|l|}
\hline Requirement & Description \\
\hline Space-time cube Method & $\begin{array}{l}\text { A methodology for generating a } \\
\text { space-time cube from transaction } \\
\text { data }\end{array}$ \\
\hline Space-time cube & An example space-time cube \\
\hline $\begin{array}{l}\text { Machine Learning } \\
\text { Program }\end{array}$ & $\begin{array}{l}\text { Machine learning program to } \\
\text { predict home values from assessor } \\
\text { data }\end{array}$ \\
\hline Analysis & $\begin{array}{l}\text { Analysis of the results of both the } \\
\text { space-time cube and machine } \\
\text { learning program }\end{array}$ \\
\hline
\end{tabular}

The second significant functional requirement of this project is the development of a machine learning algorithm that allows the user to predict home values in a given region from assessor data. This algorithm should be able to take data provided by the user, process and learn from that data, then predict home prices for homes based on their assessed features.

\subsubsection{Non-Functional Requirements}

Regarding non-functional requirements, as this project is more concerned with proving a methodology, there are only a few (Table 2). As multiple tools required for the creation of a space-time cube are Esri products, access to ArcGIS Pro is required in order for this method to be reproduced. Similarly, scripts were written in Python to generate random points as well as to create the machine learning program, so Python and some knowledge of the language are required for the scripts to be applied to new datasets beyond those implemented in this project.

Table 2. Non-Functional Requirements.

\begin{tabular}{|l|l|}
\hline Requirement & Description \\
\hline ArcGIS Pro & $\begin{array}{l}\text { To create and display a space-time } \\
\text { cube, access to ArcGIS Pro is } \\
\text { necessary }\end{array}$ \\
\hline Python & $\begin{array}{l}\text { Python language to run scripts for } \\
\text { both the space-time cube creation } \\
\text { and the machine learning } \\
\text { implementation }\end{array}$ \\
\hline Python Knowledge & $\begin{array}{l}\text { Knowledge of the Python language } \\
\text { to change the data scripts are } \\
\text { applied to }\end{array}$ \\
\hline
\end{tabular}




\subsection{System Design}

As there are two individual deliverables for this project, a space-time cube from transaction data and a machine learning program, there are two separate system designs.

To develop the space-time cube, a few system components were included (Figure 31). First, the system needs two inputs, a polygon feature of geographic boundaries for the spatial shape of the space-time cube bins and the historical transaction data containing home locations, transaction values of those homes, and the dates of those transactions.

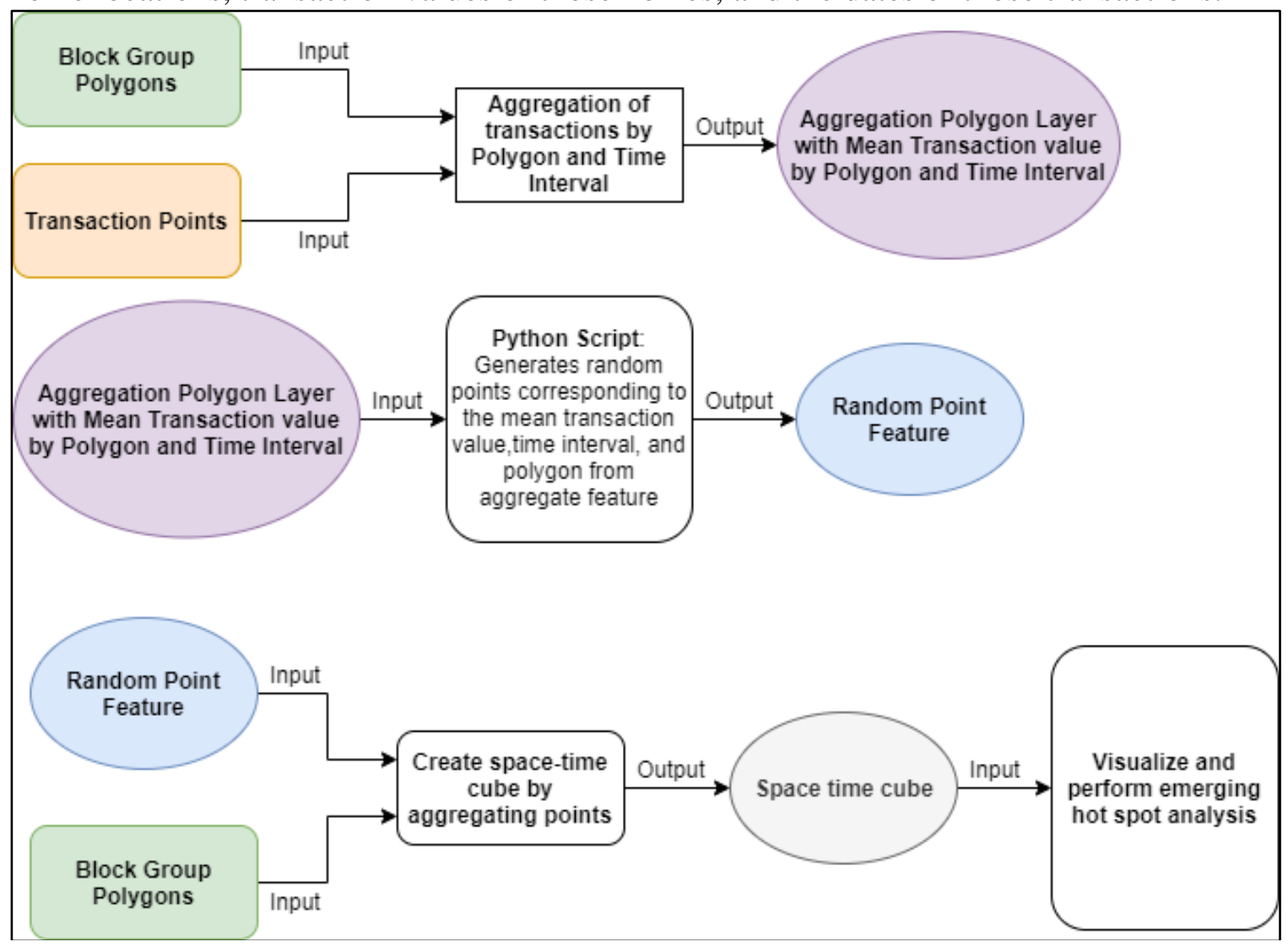

Figure 3-1: Space-time cube system design

From these two inputs, the mean transaction value by time and location was calculated within chosen geographic boundaries. From this aggregation, a random point layer generated from the aggregated mean value would be created to allow for the creation of a space-time cube. These random points, representing mean transaction values, would then be used to generate a space-time cube of mean transaction values for the region of study. Finally, an emerging hot spot analysis would then be performed on the generated space-time cube. The results of this process would be a space-time cube and a hot spot analysis of that space-time cube. These results can then be used to visually represent and statistically analyze spatio-temporal trends in home values.

The second part of this project, the machine learning portion, will have a simpler system design in which the user inputs specific assessor data into the algorithm and the resulting predictions of home values are produced. As such, this secondary system 
consists simply of the machine learning Python scripts and the data the scripts will train on and test with.

\subsection{Project Plan}

The final plan outlined for this project assumed five primary tasks as necessary for its completion. The first task involved processing and preparing the data into a format suitable for use in the creation of a space-time cube and in the training and testing of a machine learning algorithm. Since both major functional requirements of this project rely on the completion of this task, it was vital to accomplish the data processing early in the life of this project.

Once the data had been processed, the second stage was to create space-time cubes from the data. While the creation of a space-time cube is relatively simple in ArcGIS Pro, the data provided needed to be manipulated further beyond the initial data processing to ensure it worked properly with the Create Space-time cube tool.

The third task involved analyzing the resulting space-time cubes. Using the ESRI geoprocessing tool of Emerging Hot/Cold Spot analysis, insights into the spatio-temporal patterns of home values could be made. Application of this tool will be simple. However, it is vital to understand the methodology behind each tool, as well as the resulting outputs.

The second aspect of this project, machine learning models to predict home values, comprises the final two tasks. The fourth task was to create machine learning models in Python that will take a property's characteristics and predict a value for that property. To do this, the algorithms will need to be trained on assessor information provided by the client.

The fifth and final task will be to test these programs against a selected test data sample. Once applied to this test data, the results can then be analyzed for efficacy and accuracy. Depending on the results, the algorithm may need to be adjusted.

While the initial trajectory conceived for this project plan maintained throughout the life of the project, certain tasks took an unexpectedly long time to complete. In particular, processing the historical transaction and assessor data files provided became a significant undertaking and one of the most time-consuming aspects of the project. Similarly, generating the space-time cube and its various associated analyses took more wrangling than expected.

\subsection{Summary}

The purpose of this project is to provide the client with a means of visualizing and analyzing spatio-temporal trends in home values, as well as with machine learning models that can learn from assessor data and predict home values from assessor features.

To ensure the thorough completion of this project, functional and non-functional requirements were established. Functional requirements included the development of a method for creating a space-time cube from home transaction data, example space-time cubes derived from this method, machine-learning methods to predict home values from assessor data, and analysis of the results of both the resulting space-time cubes and the machine learning program. 
The project will result in two separate system designs, one for the development of the space-time cube method and one for the machine learning program. The system design for the space-time cube methodology consists of the workflow associated with the method, while the system design for the machine learning program consists of the data to be implemented in the program and the Python script for the machine learning program.

Upon completion of this project, a methodology for creating a space-time cube of historical home transaction values and machine learning models for predicting home values from assessor features will be provided to the client for future implementation and analysis. While the initial project plan's trajectory was adhered to, certain steps in the plan required more time and effort than assumed at the start of the project. 



\section{Chapter 4 - Database Design}

This chapter will describe the database design implemented for this project, as well as the processes taken to clean and prepare the data for use. Regarding the database design, Section 4.1 will detail the conceptual model of the database, and Section 4.2 will detail the logical model of the database. Regarding the data itself, Section 4.3 will describe the sources for the data, and Section 4.4 will detail the processes involved with cleaning and preparing the data for implementation.

\subsection{Conceptual Data Model}

The conceptual model of the major entities and their relationships of this project are displayed in Figure 4-1.

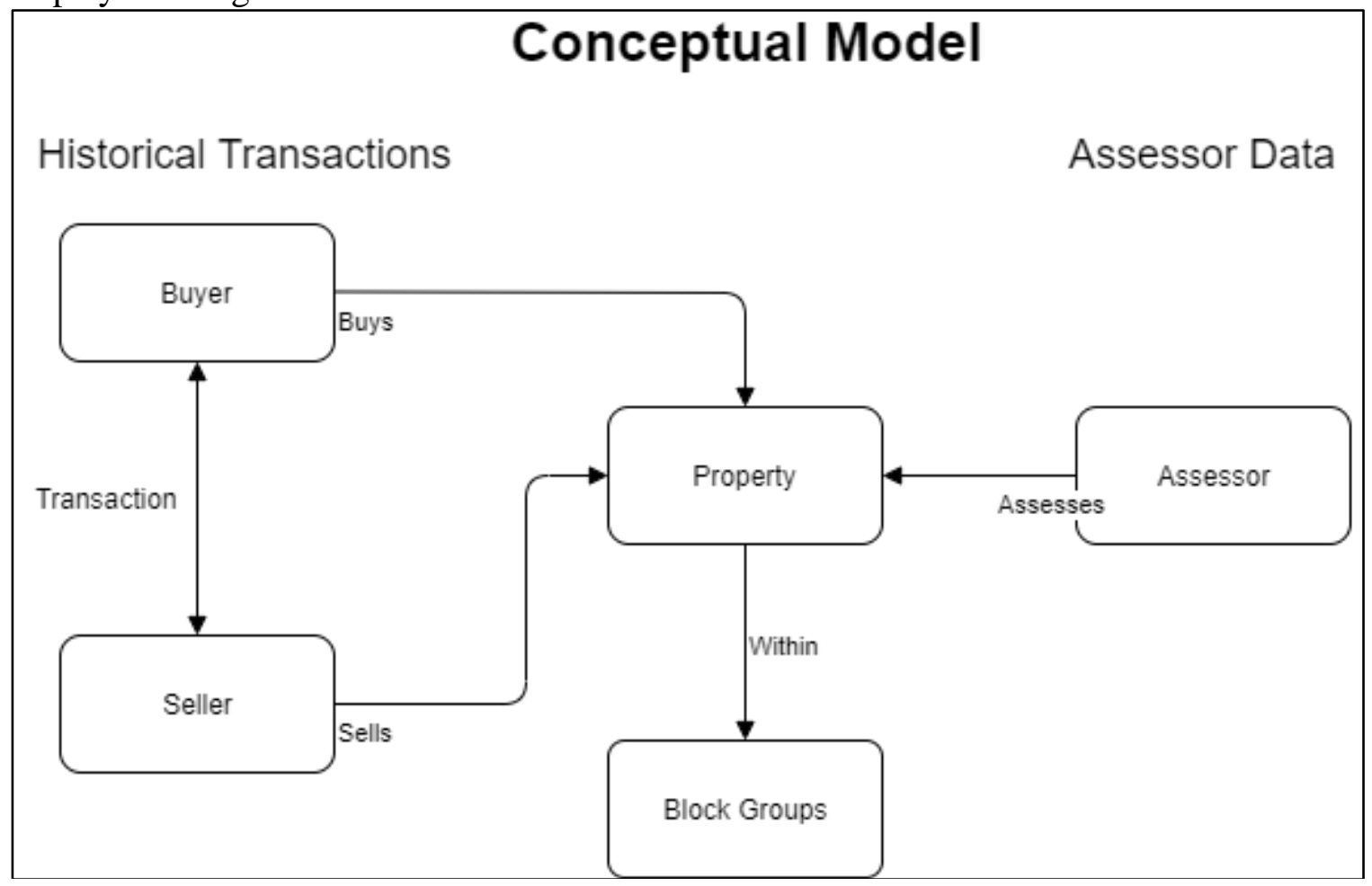

Figure 4-1: Diagram of the conceptual model of the historical transaction, assessor information, and block groups

The entities for this project consist of three main facets: historical transactions, assessor information, and block groups. Each of these facets relate to one another through a common home property. Each individual transaction involves a buyer and a seller engaging in an exchange of property rights for financial compensation. This transaction revolves around a physical property located somewhere in Southern California. This property, in turn, will have been assessed by an assessor, who establishes various characteristics of a property. Finally, as each property represents a physical location, 
those properties exist within designated Census block groups throughout Southern California.

\subsection{Logical Data Model}

The logical model displays the way that the entities from the conceptual model are implemented into a database. As the structure of the data is fairly simple, the Historical Transactions and the Block Groups tables were stored in the geodatabase as two sperate tables (figure 4-2). The transactions were implemented as a point feature class, with each point representing a location of a property involved in a transaction, and the block groups were implemented as a polygon feature class, with each polygon representing an individual block group.
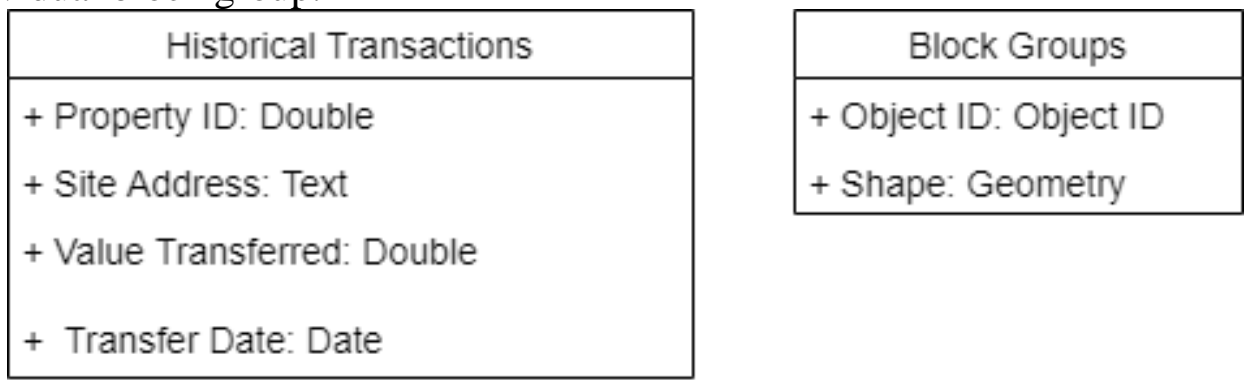

Figure 4-2: Space-time cube logical model

Regarding the Assessor Information data, as it was not relevant to the part of the project done in ArcGIS Pro, it was not stored in a geodatabase. Instead, the data were stored in a flat comma-separated value (CSV) file, so that it could later be used in the machine learning models. Attributes in the CSV file used include property ID, nine assessed features, the most recent transaction amount, and the year of the most recent transaction. The nine assessed features included ZIP code, total square footage, number of garages, lot size, number of bathrooms, number of bedrooms, , number of rooms, number of stories, and whether or not the property has a pool.

\subsection{Data Sources}

The bulk of the data used in this project was provided by the client, Dr. Moenius, who in turn purchased access to the data from CoreLogic, previously DataQuick. The data provided by the client came in fixed width text file formats. The dataset was made up of two file types, a historical transactions file and an assessor information file. The historical transaction files contain quarterly transaction information, while the assessor information files contain quarterly assessor information. The client provided access to five years of quarterly information, and therefore a total of forty text files. Along with the actual data files, the client also provided two metadata Excel files that contained the field number, field name, ANSI standard data type, SQL server datatype, precision of the field, scale of the field, length of the field, whether or not empty values were enabled for the field, and the definition of the field for both the historical and the assessor files. Additionally, a 
shapefile of block groups in California developed by the U.S. Census Bureau was retrieved from data.gov, the U.S. government's website for open data.

While the provided data contains vital information relevant to the completion of this project, the format it was provided in cannot be used directly for implementation and analysis central to this project. As such, the data needed to be altered to be applied to a space-time cube and analyzed.

\subsection{Data Scrubbing and Loading}

The data provided by the client were contained in a fixed width text file format, and as such, were not able to be accurately imported into a SQL Server database or an ArcGIS Pro Geodatabase, as the format of the data confused the automatic import, and incorrect columns, headers, and rows were produced. To address these incompatibilities, scripts were written to clean and export the data into CSV files, which could then be brought into a database and into ArcGIS Pro for geocoding, as well as be used in the Sklearn machine learning library.

The initial forty files provided were of two datasets, twenty assessor data files and twenty historical transaction data files. The assessor data files contained one hundred and seventy-nine fields of assessor information (number of bathrooms, number of bedrooms, square footage, etc.), while the historical transaction data files contained eighty-seven fields of information regarding property transactions (value transferred, date of transfer, etc.). Much of the information available in these files was not relevant to this project, and as such, many of the fields and rows were eliminated. For the assessor files, all but eighteen fields were excluded. In order to make the area of study more manageable, any rows not in San Bernardino County were removed. Also, to ensure compatibility with the machine learning models, null values in the pool column, which indicate a property as not having a pool, were changed to "N". Furthermore, as the data provided was for all real estate transactions and this project is only concerned with home values, any rows that were not of standard use code RSFR (single family residence) were removed. To make the dataset more manageable, any rows with a transaction earlier than the year 2000 were removed. As a means of introducing a temporal scaling variable into the assessor data, the median home transaction value for each year was calculated, and the corresponding value was then joined back to the assessor data. The reasoning behind introducing a temporal scaler is clarified in Section 5.2.1. Furthermore, to introduce a variable representing the age of a property for the most recent transaction, a new variable was calculated by subtracting the year a home was built from the year of the most recent transaction for the property. Finally, any rows with null values, properties with transaction values, rooms, bedrooms, lot size, bathrooms, or stories of 0 , and any rows with a number of rooms greater than three standard deviations (any properties with more than 13 rooms), a lot size less than negative three standard deviations (less than 2,400 square feet), home square footage less than negative three standard deviations (less than 552 square feet) or with a transaction value greater than three standard deviations away from the mean (any transactions higher than $\$ 1,250,000$ ) or less than negative three standard deviations ( less than $\$ 10,000$ ) were removed to avoid deleterious skewing of the data. For the historical transaction files, all but eleven fields were excluded, and any rows that were not in San Bernardino County, were missing an address, that had a null or zero transfer value, or that had a transfer value larger than $\$ 1,500,000$ were removed. 
Unfortunately, due to the unwieldiness of the data provided, the two tables could not be joined before implementation of the space-time cube. As such, in order to limit exposure from real estate transactions of property other than single-family residences, the data were visually analyzed, and from the distribution of the data, $\$, 1500,000$ was determined to be a reasonable value amount to identify any outliers.

To perform this cleaning and migration process, two Python scripts were written, one for the historical transaction data and one for the assessor data. The process for the two were largely similar, however, as the assessor dataset was particularly large, additional steps were introduced so the limited RAM available was not exceeded. To import the text files into Python data frames, the Pandas library's read fixed width function was used. This function reads fixed width files into a Pandas data frame. The text files did not contain the corresponding field headers, nor were the fields of a consistent width, and as they were text files, the datatypes needed to be specified to ensure that they were imported into the data frame properly. As such, information regarding the field headers, field length, and the field datatype needed to be read from the excel metadata files provided by the client. In regard to the datatypes, as the metadata files were created with SQL in mind, the SQL datatypes provided were read in and iterated through, with each SQL datatype being replaced by the corresponding Python datatype.

For the historical data, each quarter's text file was read in as a temporary data frame, which was then appended to the final data frame of all quarterly historical transactions. This final data frame was then exported to a CSV file, which would then be read back into a Python data frame to be cleaned. Using the Pandas read CSV function, the file was imported with only the desired fields included. Once fully imported, any values not in San Bernardino County, missing an address, or that had a null or a transfer value of 0 were removed. Upon further examination of the data, large home values and transactions with dates prior to 2014 were discovered, both of which had deleterious effects on the distribution of the data. To address this, any transaction with a date prior to 2014 or a transaction value greater than 1.5 million dollars were removed. This cleaned data frame was then exported as a final, cleaned historical transaction CSV of 162,224 rows. This file was then imported into ArcGIS Pro where it was geocoded using Esri's geocoding services.

The process for the assessor data were similar to that implemented for the historical data, but with additional steps to prevent the available RAM from being exceeded. Rather than reading each text file into a single data frame to be cleaned, each quarter was made into its own CSV. By cleaning each quarterly file individually, the amount of information stored in the computer's RAM could be kept at a manageable level, limiting the risk of crashes during the cleaning process. These CSVs were then read back into Python and concatenated into a final, cleaned data frame to be exported in a cleaned assessor CSV file of 999,475 rows. As the assessor data were not relevant to the space-time cube, the data did not need to be imported into ArcGIS Pro.

While not nearly as complex, the California census block group polygon layer also needed to be clipped to just the block groups contained within San Bernardino County. This was easily accomplished in ArcGIS Pro by using the select layer by location tool and using a San Bernardino County boundary polygon layer as a selecting feature on the block group feature. Once selected, the San Bernardino block groups could then be made into a new feature. 
After these scrubbing and loading processes were complete, the data were in a appropriate state to be used in analysis.

\subsection{Summary}

Preparing the data provided for this project was a significant hurdle to the project's completion. While the initial conceptual and logical structures of the data are fairly simple, the implementation of the data provided into useable formats involved writing Python scripts to create database friendly files, as well as to eliminate any undesirable features. 



\section{Chapter 5 - Implementation}

This chapter will discuss the processes involved in implementing the solutions for this project. It is comprised of two main sections. Section 5.1 discusses the development and implementation of a method to generate a space-time cube from historical home transaction data. Section 5.1 consists of four sub-sections. Section 5.1.1 provides an overview of space-time cubes. Section 5.1.2 describes the processing of the historical transaction data into a form suitable for being applied to a space-time cube. Section 5.1.3 describes the process of creating a space-time cube from the processed data. Finally, section 5.1.4 describes the process of visualizing and performing a hot spot analysis of the space-time cube. Section 5.2 discusses the implementation of two machine learning algorithms to predict home values from assessor data. The chapter ends with a summary in section 5.3.

\subsection{Space-Time Cube Method}

The first aspect of this project was to develop a method of visually representing and statistically analyzing spatio-temporal trends in home values. It was determined that a space-time cube would be an efficient and effective method of achieving these two requirements. This section will discuss the processes necessary to render the space-time cube construct using ArcGIS Pro.

\subsubsection{Space-Time Cube Overview}

A space-time cube is a method of visually conveying and analyzing spatio-temporal information, information that has been collected through both space and time. By utilizing these two attributes, analysts can gather insight into how trends develop in space through time. For instance, space-time cubes are often used to track crime and disease hot spots through specific time frames.

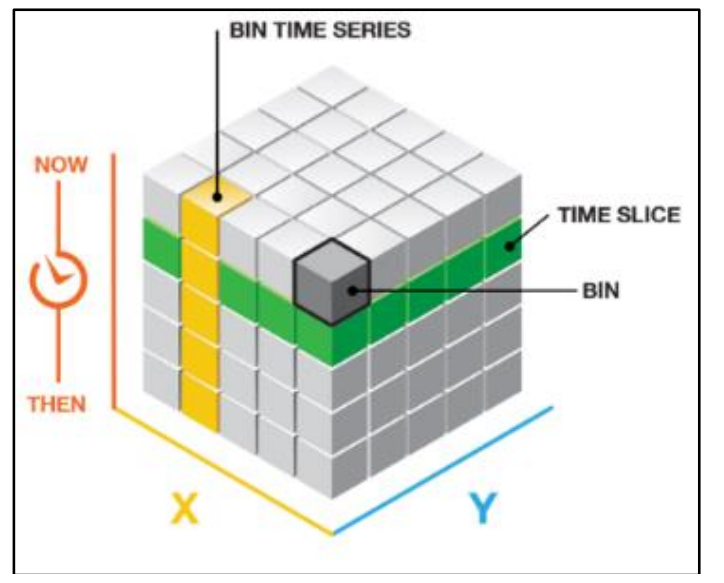

Figure 5-1: Esri diagram of a basic space-time cube (Esri b, 2019)

Figure 5-1 displays the general structure of a space-time cube generated in ArcGIS Pro. In the diagram, each individual block is a space time bin, an aggregation of events in 
a specific area for a specific time period, with the space-time cube as a whole being made up of many individual bins. Each layer of bins in the cube represents a time slice, all bins for a specific time step. For instance, if a researcher was studying yearly trends over the course of 5 years, then each time slice would represent an individual year of observations. Each column of bins represents a bin time series, all bins for one specific area for the entire time frame being studied.

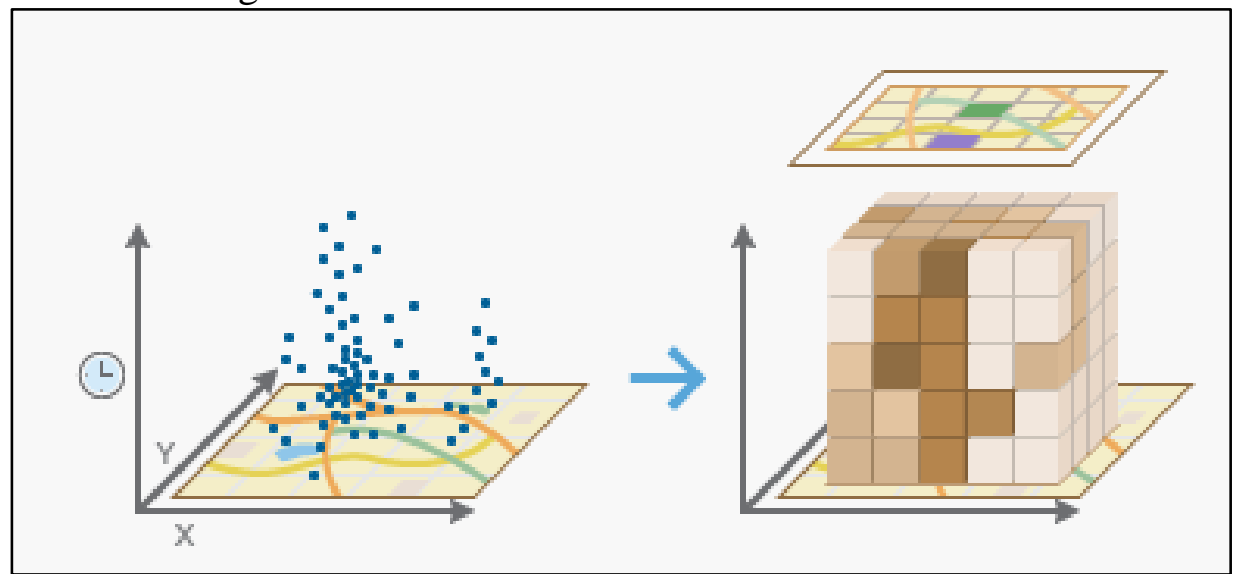

Figure 5-2: Space-Time Cube from Aggregated Points Illustration (Esri a, 2019)

Esri supplies two methods of implementing a space-time cube. The method utilized by this project, Create Space-time cube by Aggregating Points, is illustrated in figure 5-2. This method will aggregate points into space-time bins and produce trends from the count of points in each of those bins, with darker bins having a higher count of points and lighter bins having a lower count of points (Esri a, 2019). The space-time bins are structured in a netCDF (Network Common Data Form) data format, a file format designed to handle "storing and retrieving data in the form of arrays" (Unidata, 2019). Due to the schema of the data provided by the client, in order for the Create Space-time cube by Aggregating Points tool to be applied, the data needed to be transformed into an appropriate form.

\subsubsection{Processing Data for Space-Time Cube Implementation}

While a point feature in its initial form, the historical transaction data provided by the client would not provide a representation of transaction value when applied to a spacetime cube. Instead, any resulting space-time cube would display the spatio-temporal trends of the number of transactions in each block group by quarter, essentially the frequency of transactions through space and time.

In order for the data provided by the client to be applied to the Create Space Time Cube by Aggregating Points tool available in ArcGIS Pro, a method needed to be created to allow for the mean transaction value of each block group to be represented as points. A visualization of the process developed for this purpose is displayed in Figure 5-3. To start this process, the time property of the historical transaction point feature needs to be activated and set to use the transaction date attribute. This can be done in the feature properties under the time pane. Once this is done, the aggregate points tool available in ArcGIS Pro can be used to aggregate the historical transaction point feature by block group and quarter, from quarter 1 of 2014 to quarter 3 of 2018 (19 quarters). The result of 
this aggregation is a polygon feature containing 19 layers of polygons, with each layer being comprised of the corresponding mean transaction value of each block group by quarter. Figure 5-3 shows an example of the mean value of home transactions in the third quarter of 2018.

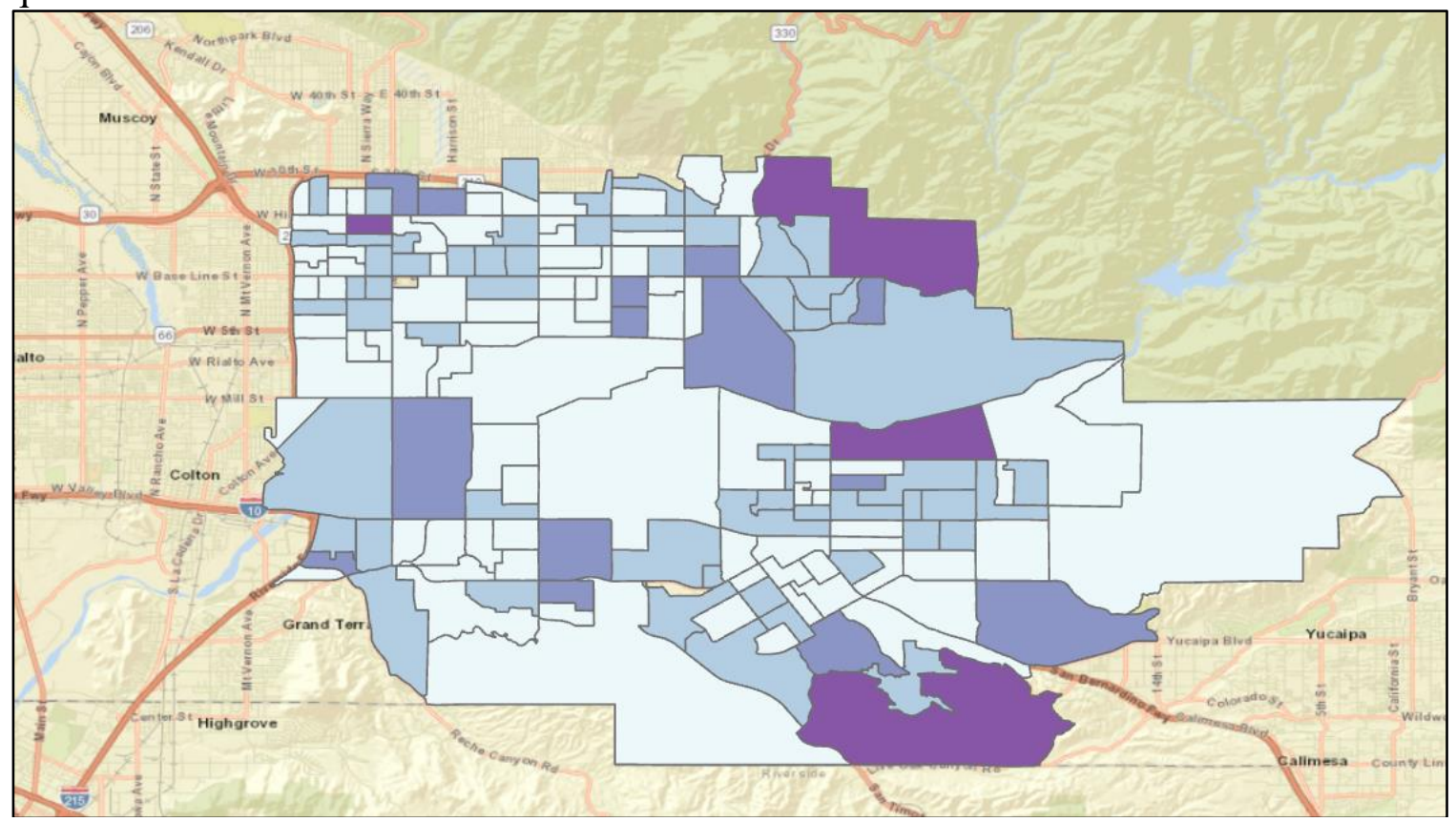

Figure 5-3: Aggregated polygon feature for the third quarter of 2018

Because the space-time cube aggregation relies on point data, these mean transaction values then needed to be translated back into a point feature. To achieve this, a Python script was written that would take the aggregated mean transaction value for each quarter-block group and generate a corresponding number of random points. However, to keep the number of points generated at a reasonable amount, a point value of $\$ 10,000$ was chosen, and the mean transaction value of each quarter-block group was then divided by this point value. As displayed in figure 5-4, for a hypothetical block group in quarter 1 , if the mean transaction value was $\$ 100,000$, then the number of points to be generated for this block group would be 10 points, each of which essentially represents $\$ 10,000$. 


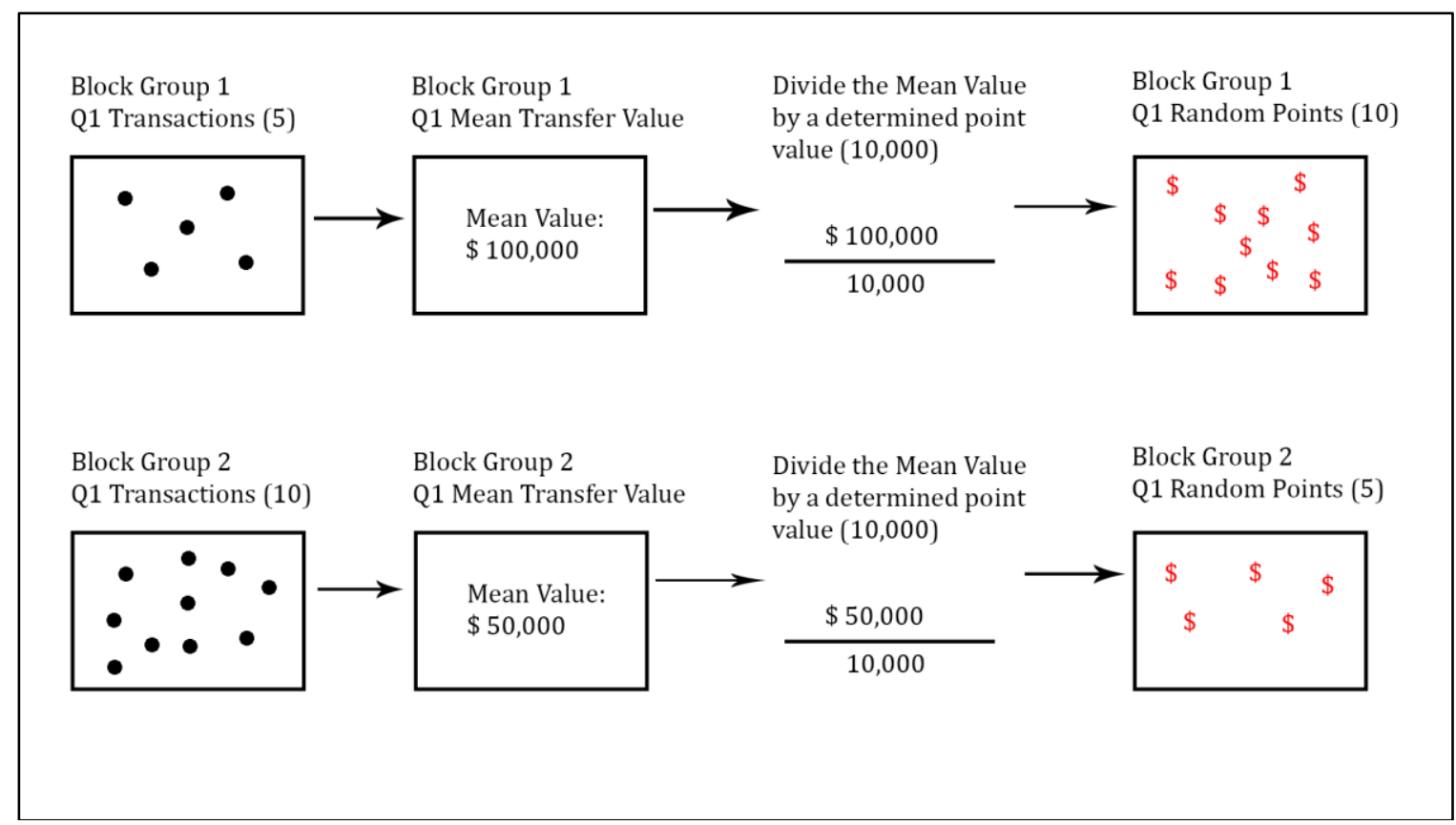

Figure 5-4: Diagram of mean transaction point generation

The Python script written to perform this task performs three major functions. The first of these functions is to create two new fields that are added to the aggregated points feature, a field for the random points to be calculated and a field for a simplified date field that is easier to work with in Python. After being added, the two fields are then calculated from existing fields. The random points field is calculated by dividing the mean transaction value of each aggregated polygon by 10,000, the chosen point value. The date field is calculated by splitting the start date time field so that just the yyyy-mmdd date format is kept. The random points field is of type long, the date field is of type string. Once these fields are calculated, the script then runs through each polygon in the aggregated point feature and generates the appropriate number of random points for the corresponding polygon. Each point generated also has the two fields added containing the appropriate time interval and polygon ID. The script results in a final random point feature with five attributes: OBJECTID, SHAPE, CID, date, and poly_id.

The resulting point feature is almost ready to be implemented into a space-time cube. However, as the space-time cube tool operates in a 3D local scene, the point feature and block group polygon feature need to be projected into a projected coordinate system. As the two areas of study chosen for this project are both in San Bernardino County, the NAD 19832011 State-Plane California V coordinate system was chosen. Once projected, the two features are ready to be implemented into a space-time cube.

\subsubsection{Implementing Space-Time Cube}

After creating the appropriate mean transaction point feature, a space-time cube can now be generated. The Create Space-Time Cube by Aggregating Points tool takes several input parameters: an input point feature, the time field, the desired time-step interval, the time step alignment, and the aggregation type. For this project, the input point feature is the random point feature generated in the previous section, and the time field is the date 
field within the point feature. The time step interval determines how points are aggregated through time. For instance, a time interval could be one day, one month, or three years. This project chose to aggregate transactions by quarterly three-month intervals from quarter 1 of 2014 to quarter 3 of 2018, for a total of 19 quarters. The timestep alignment determines how data was aggregated through time, either from the first date in the dataset or from the end date. This parameter was important for handling temporal bias in how data is collected, but as the data in this project was already of a defined interval of three months, this parameter is not important and can be set to the default of end-time. Finally, the aggregation shape type can either be a regularly shaped grid or derived from defined locations. The aggregation shape type chosen for this project are the defined locations of block groups, a geographic census unit. Two areas of study were chosen for example space-time cubes to be generated of, the Redlands and Chino areas of Southern California, as seen in figures 5-4 and 5-5.

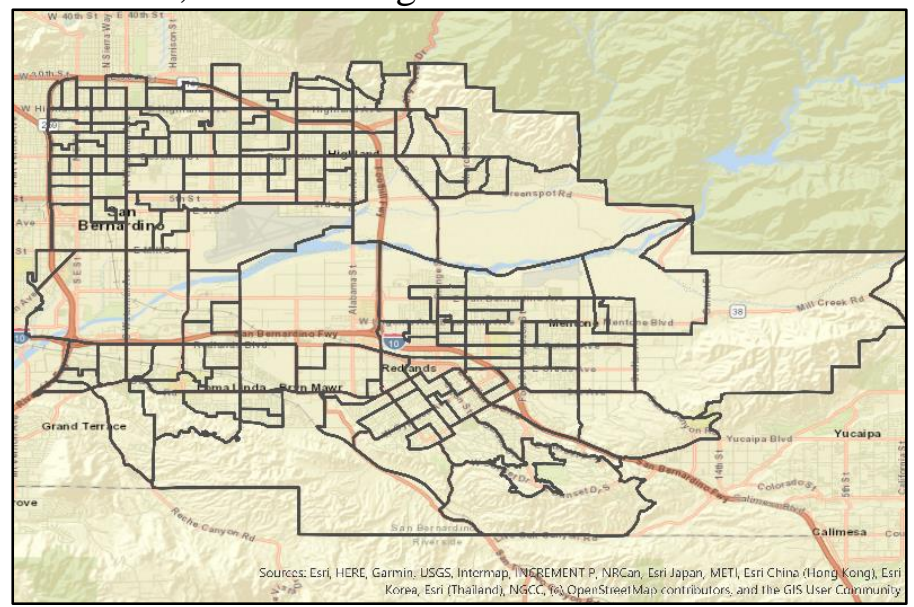

Figure 5-4: Block groups for the Redlands area

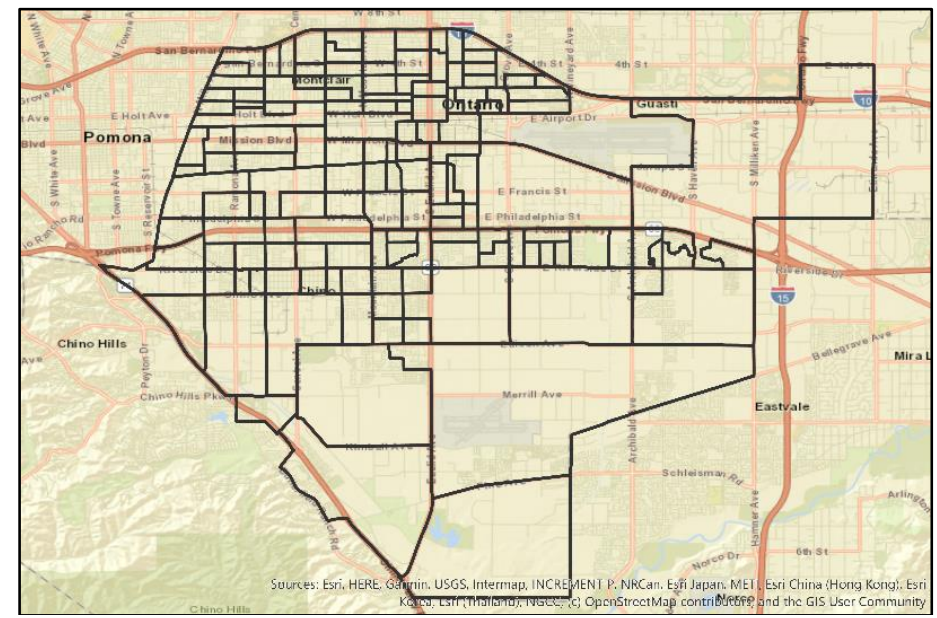

Figure 5-5: Block groups for the Chino area

Taken together, the time step interval and aggregation shape parameters determine the spatio-temporal dimensions of each individual space-time bin in the space-time cube. The final space-time bin dimensions for this project's space-time cube are therefore quarterly block group aggregates. With these parameters, a space-time cube can be 
generated; however, in order to display and perform an analysis on the space-time cube, additional steps must be taken.

\subsubsection{Visualizing and Performing Hot Spot Analysis}

Upon creation of a space-time cube, a netCDF file is created in the chosen geodatabase. However, the space-time cube will not be automatically added to a map or scene. To display the space-time cube, the visualize space-time cube tool must be run.

Similar to the visualization processes, a hot spot analysis of the space-time cube can be performed to identify any spatiotemporal trends present in the observed data. To identify hot and cold spots, Esri's Emerging Hot Spot Analysis tool identifies features in the data that contain high or low values and are surrounded by similarly high or low values. To calculate the significance of clusters, the tool implements the Gi* statistic (Getis and Ord, 1992). This statistical method calculates and returns a z-score for each feature in the dataset, with statistically significant positive z-scores representing high value clustering (hot spots) and statistically significant negative z-scores representing clustering of low values (cold spots).

There are two outputs of the hot spot analysis tool, one that displays results in 2D and defines 17 different kinds of trends, and the other that displays its results in 3D and defines 7 bin types for cold spots, hot spots, and bins with no significant trend. The 3D display is generated by visualizing the $2 \mathrm{D}$ hot spot analysis in $3 \mathrm{D}$, similar to how the space-time cube is generated in 3D. Both methods can provide useful insights into the spatio-temporal trends throughout a space-time cube. The analysis of the outputs will be elaborated in Chapter 6.

\subsection{Machine Learning}

The second component of this project involved developing machine learning algorithms for home value prediction and testing them for efficacy. As a large part of implementing a machine learning algorithm involves the preparation of data, much of the implementation process is described in Section 4.4. As described there, the assessor data were reduced to 10 attributes relevant to predicting a home's value. However, there was additional data preparation that needed to be done before implementing the machine learning models.

\subsubsection{Pre-Processing the Assessor Data}

Beyond scrubbing and cleaning the data, a pre-processing phase was necessary before applying the machine learning algorithms. This pre-processing phase involved creating a temporal scaler variable, encoding categorical data, creating dummy variables from the encoded categorical data, splitting the dataset into a training set and a testing set, and in the case of the Random Forest Regression, scaling the data.

To ensure that the machine learning models created by this project would work not only to predict home values in the present, but the values of homes into the future, a new temporal scaling variable needed to be created. To achieve this, the median transaction value of each year, from 2000 to 2017, was calculated. These values were then inner joined back to the entire dataset on the transaction year column. With this new median 
transaction variable, users can multiply the most recent median transaction value in the data by the predicted growth rate in home value for the current year. By doing this, the machine learning models will be able to predict the values of data with temporal properties that they have not been trained on.

Dummy variables are a method of transforming categorical variables into a form that is compatible with the mathematical equations inherent to machine learning. This method involves two steps. The first step is to encode the categorical variables in the data.

Encoding involves giving each distinct value in a categorical variable a unique numeric value. In the Python scripts written for this project, the Sklearn library was utilized, and in particular for the encoding process, the OneHotEncoder module was used. For instance, in the assessor data, the pool variable consists of two values, either Y or N. In order for these categorical values to be applied to the machine learning algorithm, they needed to be translated into numeric values. In this case, $\mathrm{Y}$ was translated into 0 and $\mathrm{N}$ was translated into 1 . However, while the two categorical values are now represented numerically, the numbers still operate as categorical values as 0 and 1 cannot be directly compared. In other words, 0 representing that a property does have a pool is not quantitatively smaller than 1 representing that a property does have a pool. This is where the dummy variables are implemented. To ensure that the machine learning algorithm is not confused by the encoded numerical values, additional columns are created and appended to the dataset. In the case of the pool variable, two columns were added, one for $\mathrm{Y}$ (representing that a property does have a pool) and one for $\mathrm{N}$ (representing that a property does have a pool). Then, each column is filled with a 1 or a 0 . The 1 and 0 essentially act as switches, with 1 meaning that column is "on" (property does have a pool) and 0 being "off" (property does not have a pool). Finally, to ensure there is no bias in the data resulting from multicollinearity amongst the created dummy variables, one of the dummy variables was removed from the data.

An additional step was introduced to provide a numerical measure of the recency of the year a home was built and the year of the most recent transaction. To achieve this, a new attribute was calculated by subtracting the year a home was built from the year of the most recent transaction for the property. By introducing this independent variable, the impact of the age of a home on the transaction amount is able to be quantified and applied as numeric variable.

Once all categorical variables had been encoded, the corresponding dummy variables had been created, and the new variable of the age of the property was introduced, the data were separated into a training dataset and a testing dataset. When performing supervised machine learning, the algorithm is trained on designated training data that contains inputoutput pairs (independent variables - dependent variable pairs). For the program in this project, twenty percent of the data were segregated for use as testing data, while the remaining eighty percent was used for training. The dependent variable to be predicted is the value of the most recent transaction, essentially the most recent price of the home, and the independent variables are the assessor features of the home. They are the ZIP code, garage type, lot size, number of bathrooms, number of bedrooms, number of rooms, number of stories, pool code, square-footage, age of the property, and the median transaction value for the most recent transaction year. From this training data, the machine learning algorithms developed functions that were then applied to the 
independent variables in the testing data. The results of those predictions were then compared to the actual property value to determine the accuracy of the models.

An additional step, feature scaling, was necessary for the Random Forest algorithm, but not the Multiple Linear Regression model, as it is automatically applied in that method. Feature scaling is also known as normalization, and it is the process of adjusting the values of independent variables with different ranges to a common scale. For instance, in the assessor dataset, the largest value for square footage is 97,700 , while the largest value for number of bathrooms is 15 . If these two independent variables were introduced into the machine learning algorithm without being normalized, then the difference of scale would result in the square footage of a house having a very large influence over the dependent variable and the number of baths being rendered nearly meaningless.

Once the pre-processing of the data were completed, the two different machine learning algorithms could be applied.

\subsubsection{Implementing the Machine Learning Models}

This project chose to implement and compare two machine learning algorithms: Multiple Linear Regression and Random Forest regression. As with the preprocessing phase, the Sklearn Python library was used for each algorithm. Once the two were implemented, the results were compared to determine which method was more accurate.

When implementing the Multiple Linear Regression, a backward elimination process was implemented to ensure that the model was only built from independent variables with a significance level less than .05. Once the significance level was chosen, the backward elimination process would fit the full model with all independent variables and calculate a p-value for each. The model would then select the predictor with the highest $\mathrm{p}$-value and compare it to the significance level of .05. If the p-value was greater than .05 , then that predictor was removed. This process would then repeat until the predictor with the highest p-value was within the significance level of .05. Once this process was finished, the machine learning model was then ready for implementation.

The second model, Random Forest Regression, is more complex than the Multiple Linear Regression model and involves ensemble learning, the use of multiple algorithms or multiple iterations of the same algorithm together to create a more powerful predictive model. For this project, the Random Forest Regression model implements many iterations of decision tree regressions. A decision tree regression is a process that splits the entire dataset into optimal data subsets, and once a new variable is introduced, it is fitted into the appropriate subset, where the average of that subset is then applied to the new variable. A random forest regression segments this process further by picking $\mathrm{k}$ number of data points from the training dataset and creating a decision tree regression from this $\mathrm{k}$ number of data points. This process is then repeated $\mathrm{N}$ number of times for a total of $\mathrm{N}$ decision tree regressions, or a forest of decision trees. For this project, 200 trees were created, as 200 is considered a sufficient number of trees for maintaining a balance between accuracy and efficiency. Finally, when a new variable is introduced to be predicted, it is predicted by each of the 200 trees, with it being assigned the average predicted value across all trees. While this project ultimately decided to perform the RFR model ten times and calculate the average summary statistics of all iterations, the RFR model was also tested with 2000 trees and only one iteration. As an RFR inherently 
introduces random iteration in the process of its prediction, the purpose of the ten iterations and average results could theoretically have been achieved through one iteration of 2000 trees. However, after performing both methods of implementation, the method of ten iterations with 200 decision trees performed better than the single iteration with 2000 decision trees.

Due to the large size of the dataset, running each method on the entire dataset would take a prohibitively long time. To address this problem, each model was run 10 times on a random selection of $\mathrm{n}$ rows from the entire dataset, and the Mean Absolute Error (MAE), Root Mean Square Error (RMSE) and R-squared (R2) were calculated for each iteration. The average of the MAE, RMSE, and R2 of each model was then taken for comparison of the efficacy and accuracy of each model.

\subsection{Summary}

There were two main components to be developed and implemented in this project, a space-time cube of home values and a machine learning algorithm for predicting home values from assessor information. To implement the space-time cube, a methodology was created to translate historical transaction information into a form suitable for the spacetime cube creation tool. This methodology involved the creation of a Python script to generate random points within block group polygons to represent the mean transaction value for that block group for a specific time interval. Once this script was run, two space-time cubes were created, visualized in 3D, and applied to an emerging hot spot analysis in both 2D and 3D. The second portion of the project involved pre-processing data into a suitable form for machine learning algorithms, as well as the implementation of two different machine learning algorithms, a Multiple Linear Regression model and a Random Forest Regression model. To keep the runtime of each algorithm reasonable, random samples were taken from the entire dataset, and each model was run 10 times. Statistics were calculated from the results for comparison and evaluation of the accuracy of each model. 



\section{Chapter 6 - Results and Analysis}

This chapter will discuss the results of the project implementations covered in the previous chapter, as well as the successes and shortcomings of the methods. Section 6.1 will cover the results of the methodology developed for the implementation of the spacetime cube, as well as the difficulties experienced in achieving it. Section 6.2 will cover the results of the implementation of two different machine learning algorithms, as well as the difficulties faced in their implementation.

\subsection{Space-Time Cube Results}

In order to gain a better understanding of how the methodology designed in this project worked, two different areas were chosen for the implementation and creation of a spacetime cube. The first area, pictured in Figure 6-1, was of the area around the city of Redlands, California, and includes parts of San Bernardino, Loma Linda, Mentone, and Highland. The second area chosen, pictured in Figure 6-2, was of the area around Chino, California, and included parts of Ontario and Montclair.

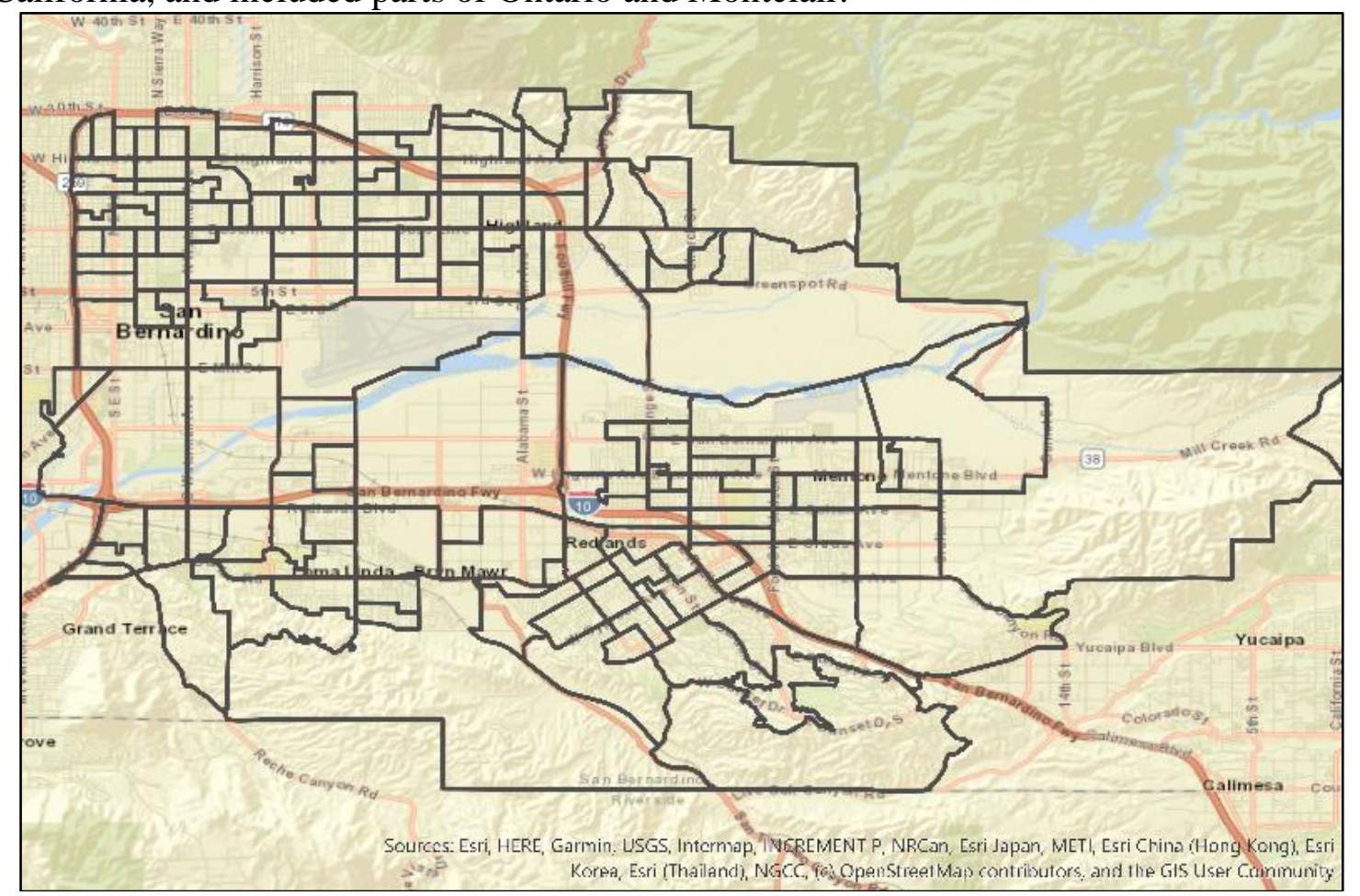

Figure 6-1: Block groups for the Redlands area 


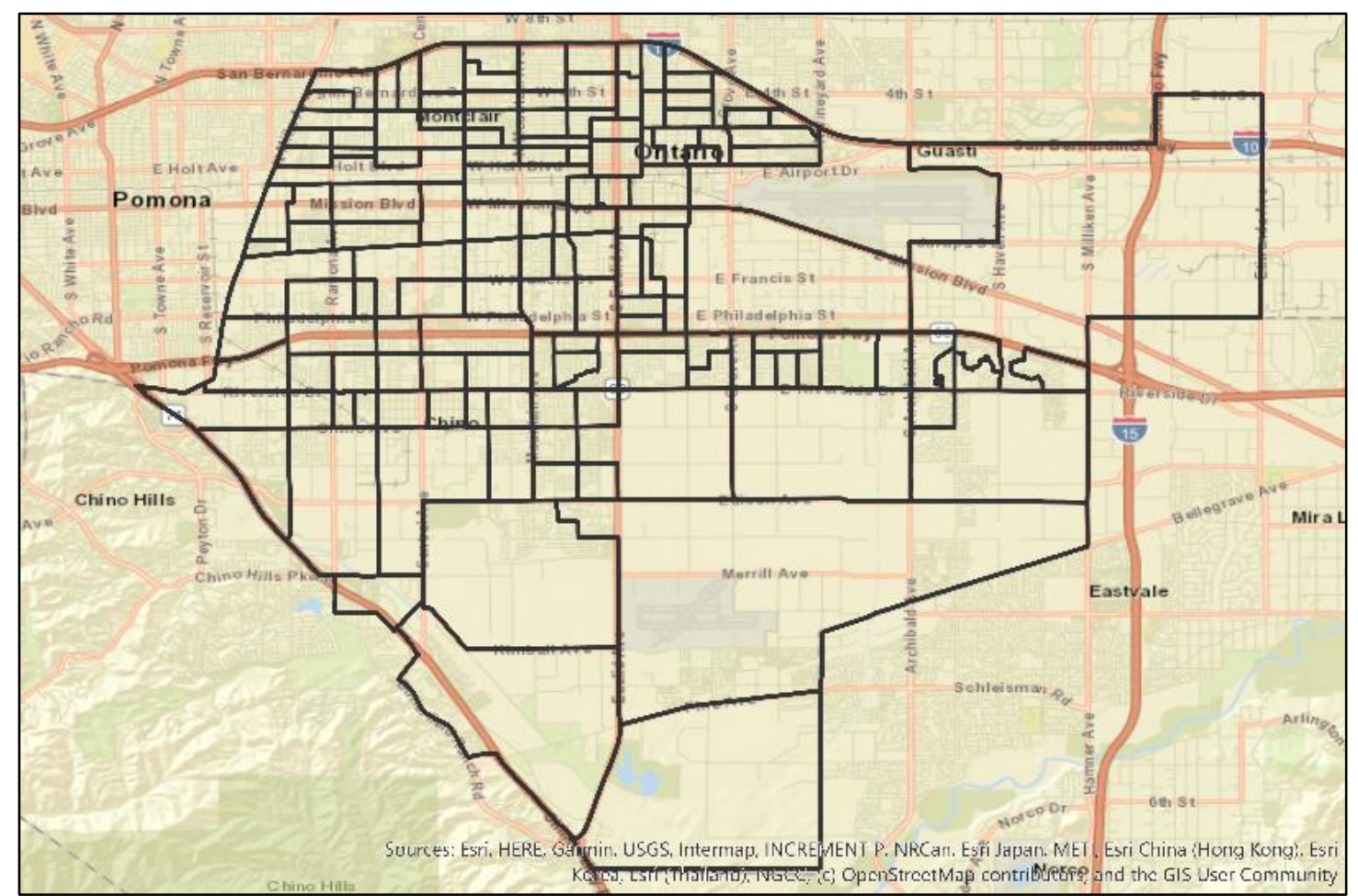

Figure 6-2: Block groups for Chino area

\subsubsection{Visualizing Space-Time Cube in 3D}

Once the space-time cubes were created, they were then visualized through two different methods. The first method, Visualize Space-time cube in 3D, results in a threedimensional render of a chosen variable and theme. In the examples provided in figures 6-3 and 6-4, the variable and theme chosen are the counts of aggregated points in each space-time bin, with the points representing the mean transaction value. Visually, this means that space-time bins with a darker shade of blue contain a larger count of points, and therefore a higher mean transaction value. Conversely, space-time bins with a lighter grey color have a lower mean transaction value. Finally, space time bins where there were no transactions for that particular block group/time-interval pair are visualized as a transparent bin. 


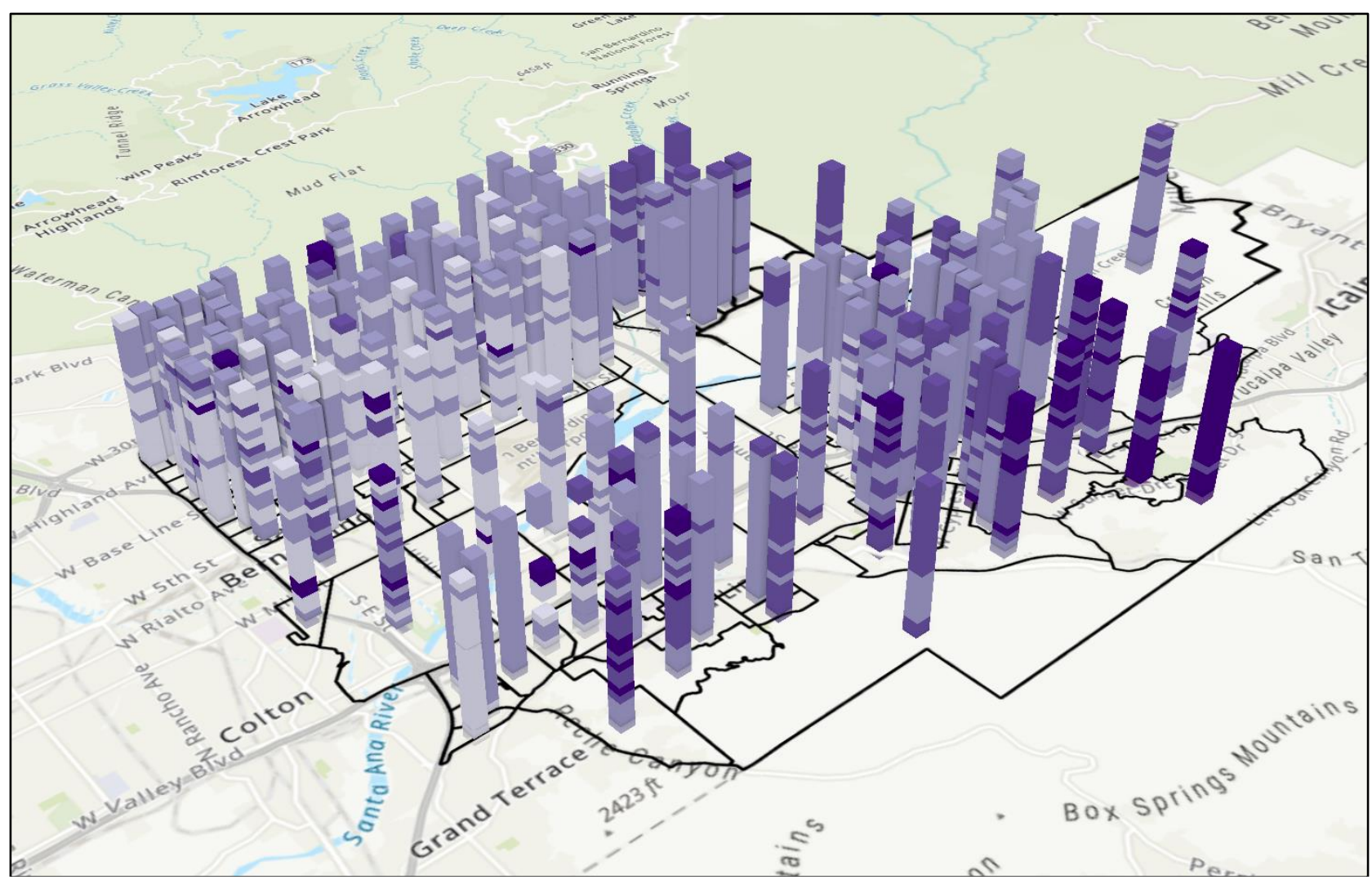

Figure 6-3: 3D Space-Time Cube of mean transaction value by block group/quarter from Q1 2014 - Q3 2018 for the Redlands area 


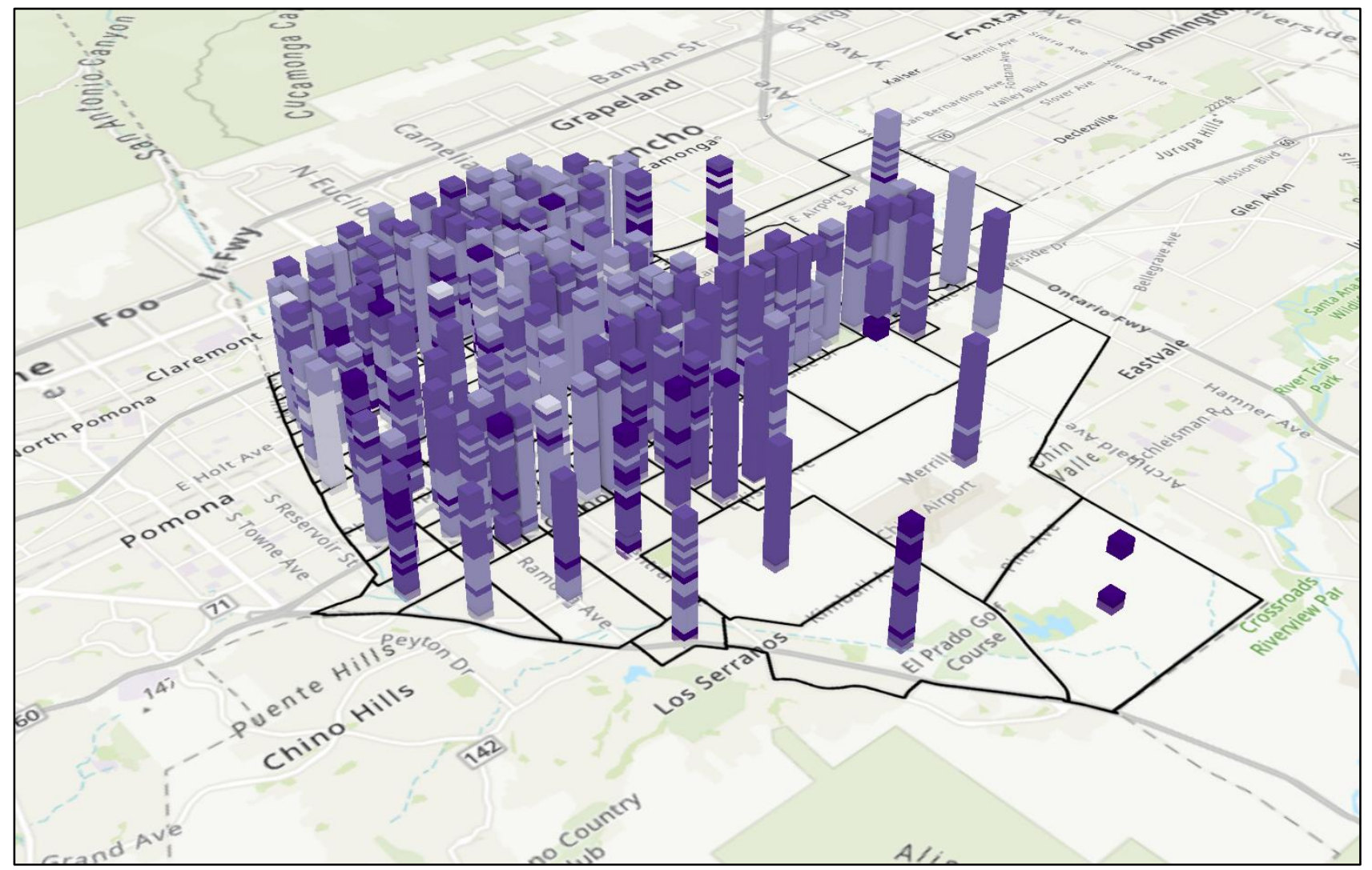

Figure 6-4: 3D Space-Time Cube of mean transaction value by block group/quarter from Q1 2014 - Q3 2018 for the Chino area

From these visualizations, it can be seen that the Chino area has a more homogenous pattern of high value transactions, while the Redlands area has a more heterogeneous pattern with concentrations of areas with higher transaction values segregated from areas with lower transaction values. This method of visualization addresses the first goal of the space-time cube for the client, in that it allows for the values of different spatial locations for different time intervals to be compared at once. Referring back to Figure 2-1, the client's previous method of visualizing spatio-temporal trends required an animation to walk through each individual time interval, with only one interval viewed at a time. Due to this limitation, comparison between time intervals was difficult and tedious. Now, with the implementation of a 3D visualized space-time cube, each individual space-time location can be compared to all other space-time locations at once, making visual analysis much easier. While this is an effective method of analysis on its own, a statistical approach allows for even greater insights to be made from the data.

\subsubsection{Performing 2D Hot Spot Analysis}

The other method of visualization utilized in this project consisted of producing $2 \mathrm{D}$ and 3D hot spot analyses for both of the study areas. Taken together, the results of the two tools allow the user to detect significant clustering of hot (high-value) and cold (lowvalue) spots through space and time. The $2 \mathrm{D}$ emerging hot spot analysis produces 17 
different categories, as seen in figure 6- 5, each describing a different trend of locations through time.

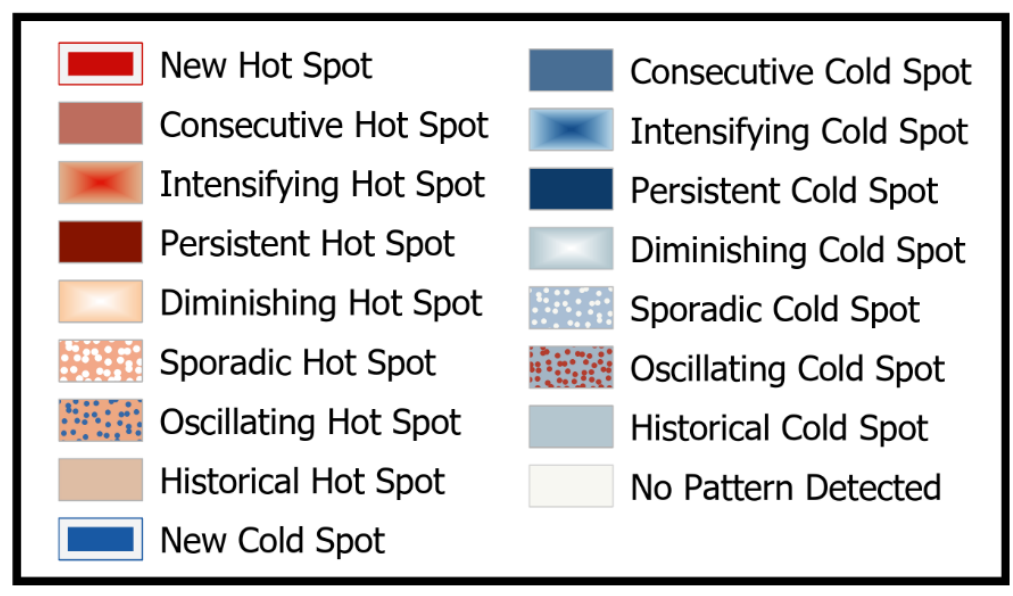

Figure 6-5: 2D Hot spot analysis legend

The results of this tool can be seen for the Redlands area (Figure 6-6) and the Chino area (Figure 6-7). Comparing the resulting hot spot analyses of the two areas shows the Redlands area as having a greater variety of spatio-temporal patterns than that of the Chino analysis. Eight different significant spatio-temporal trends can be seen in the Redlands area of study (new hot spots, consecutive hot spots, intensifying hot spots, a persistent hot spot, an oscillating hot spot, diminishing cold spots, and sporadic cold spots), while only four significant spatio-temporal trends can be seen in the Chino study area (consecutive hot spot, sporadic hot spot, sporadic cold spot, and new hot spot). 


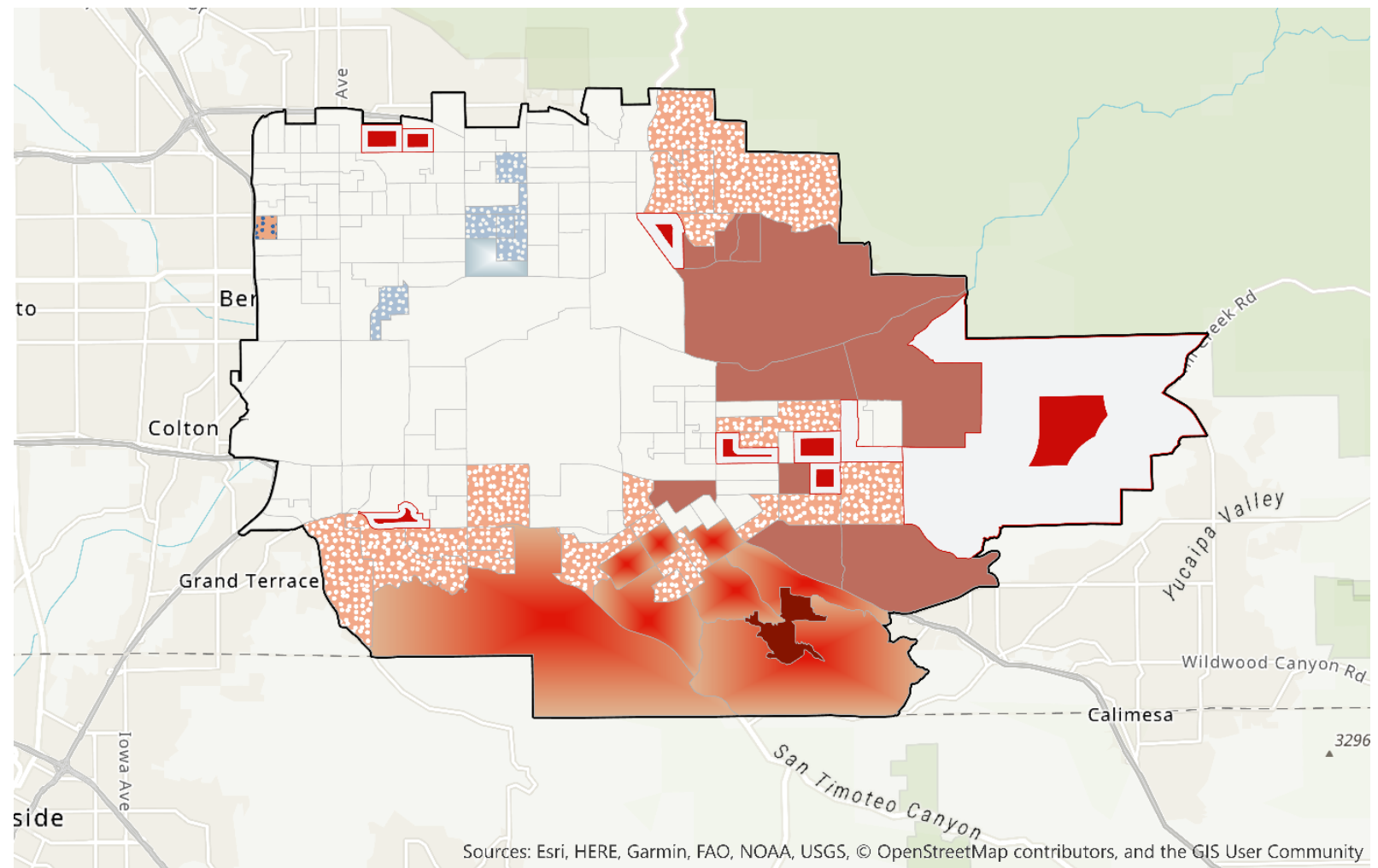

Figure 6-6: 2D Hot spot analysis of mean transaction value by block group/quarter from Q1 2014 - Q3 2018 for the Redlands area 


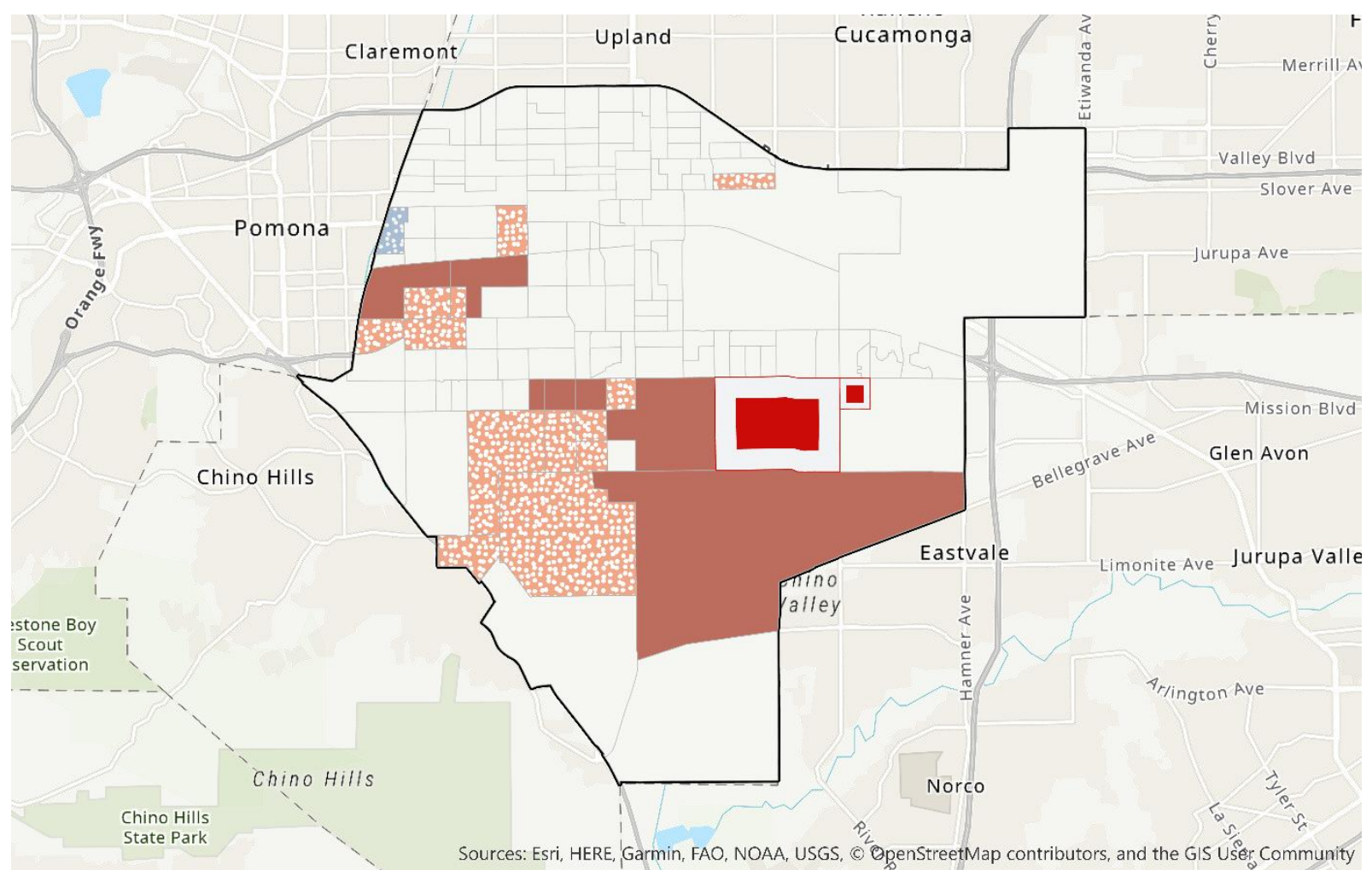

Figure 6-7: 2D Hot spot analysis of mean transaction value by block group/quarter from Q1 2014 - Q3 2018 for the Chino area

When looking at the emerging hot spot analysis of the Redlands area (Figure 6-6), some particular trends stand out. For instance, as mentioned in the previous section, the southern part of Redlands has a high concentration of high value transactions. However, when examined for significant spatio-temporal trends, there was only one area of persistent levels of high value transactions. Instead, much of the South Redlands is seen to be experiencing intensifying high-value transactions. A location categorized as an intensifying hot spot is defined by Esri's emerging hot spot tool as being a statistically significant hot spot for $90 \%$ of time intervals and as having statistically significant increases in point clustering (Esri c, 2019). For this particular project, as the points aggregated represent the mean transaction value for a particular bin, an intensifying hot spot indicates a location that has consistently had high mean transaction values and that the mean transaction value is increasing at a significant rate. Potential economic factors that can explain these intensifying hot spots are that the average annual salary of Redlands increased from $\$ 43,455$ in 2013 to $\$ 49,018$ in 2017 , the total number of jobs in the Redlands area increased from 34,163 in 2014 to 41,609 in 2017, and until 2018, permits issued to build new single-family residential units were low (Southern California Association of Governments, pp. 28, 24, 12). Taken together, the increased demand for housing not being met by an appropriate supply of new housing may have consistently driven the value of homes in the wealthy South Redlands region. 


\subsubsection{Performing 3D Hot Spot Analysis}

While the 2D method of visualizing a space-time cube allows for understanding trends present in the data over the entire time frame, the 3D method of visualizing a hot spot analysis allows the user to view individual hot and cold space-time bins for more specific observations. The 3D visualization of the hot spots produces seven types of bins, as seen in figure 6-5, three corresponding to hot spots of different confidence levels, three corresponding to cold spots of different confidence levels, one corresponding to bins with no significant spatio-temporal trend, and bins with no transactions are again transparent.

$$
\begin{array}{|ll|}
\hline & \text { Hot Spot - 99\% Confidence } \\
\text { Hot Spot - 95\% Confidence } \\
\text { Hot Spot - 90\% Confidence } \\
\text { Not Significant } \\
\text { Cold Spot - 90\% Confidence } \\
\text { Cold Spot - 95\% Confidence } \\
\hline
\end{array}
$$

Figure 6-8: 3D hot spot analysis legend 


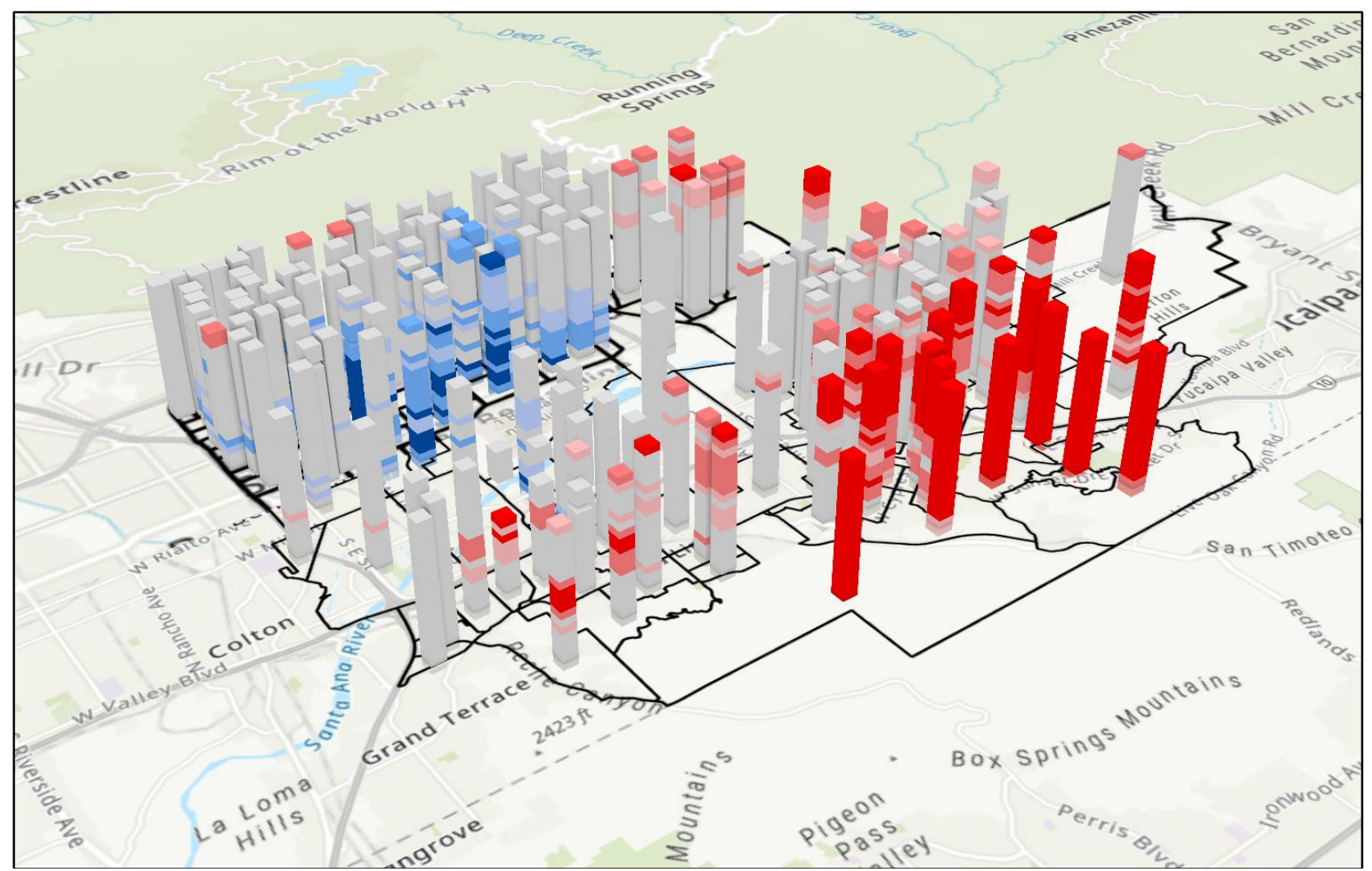

Figure 6-9: 3D Hot spot analysis of mean transaction value by block group/quarter from Q1 2014 - Q3 2018 for the Redlands area 


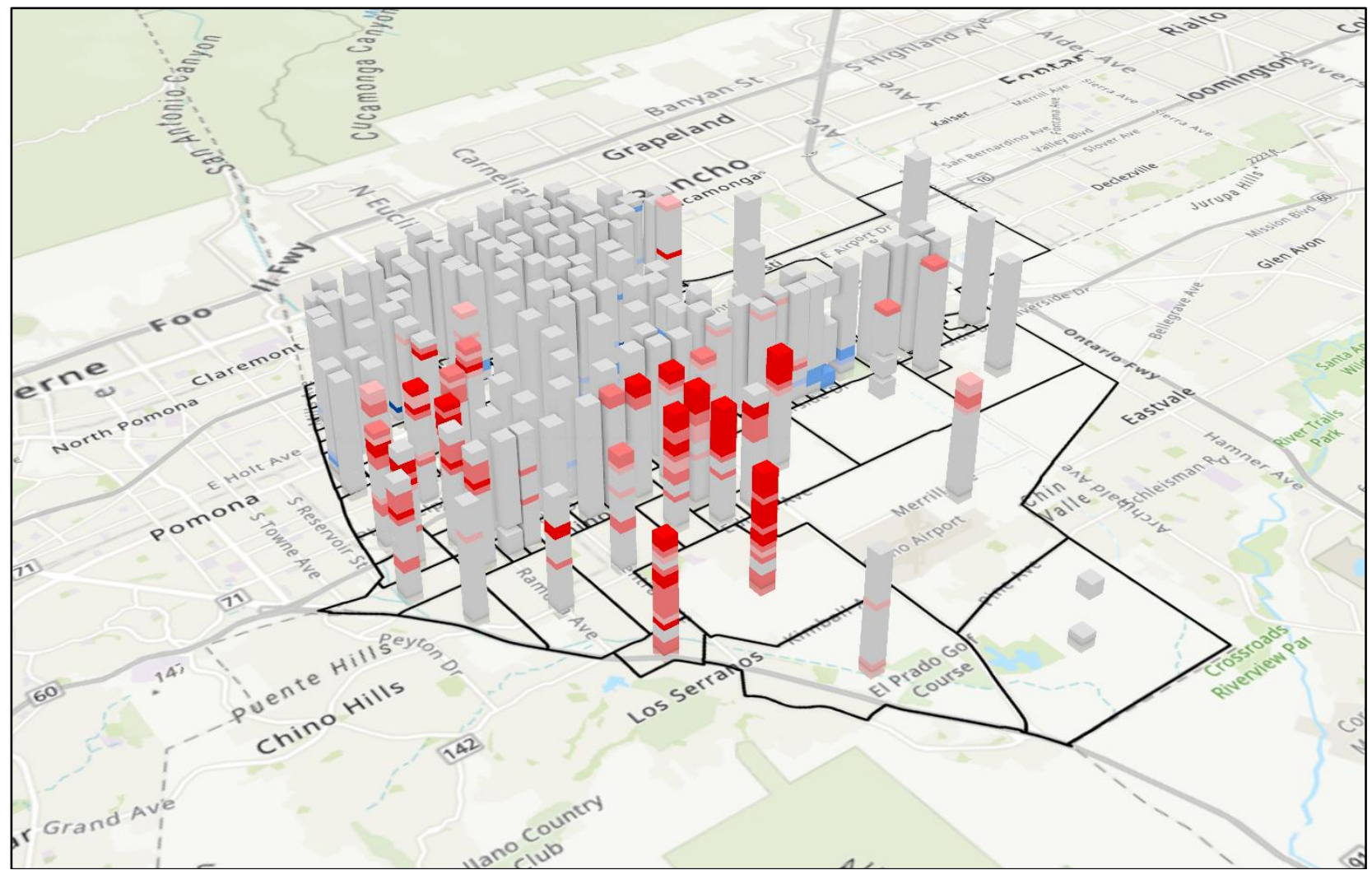

Figure 6-10: 3D Hot spot analysis of mean transaction value by block group/quarter from Q1 2014 - Q3 2018 for the Chino area

Comparing the two results, larger swathes of the Chino study area appear to have no significant trend, while the Redlands study area, similar to the results of the 3D count aggregation results, show segregated areas of hot and cold spots. These results make sense, because as seen in the 3D aggregate count space-time cubes of Figures 6-3 and 64, the Chino study area has a more homogenous pattern of high and low value areas, while the Redlands area is heterogenous with high values clustered in the southern part of Redlands and low values clustered around North Waterman Avenue and Baseline Street in San Bernardino.

Furthermore, when both 3D and 2D emerging hot spot analyses are viewed together, the overall trends detected in the 2D analysis can be further examined at the individual space-time bin level in the 3D analysis and vice-versa. For instance, as mentioned in the previous section, South Redlands is classified as intensifying hot spots, locations that are statistically significant hot spots for $90 \%$ of time intervals and have statistically significant increases in point clustering. Without this insight, when solely viewing the 3D hot spot analysis, this same area could be seen as only being a persistent hot spot, rather than a persistent hot spot with increasing values over time.

\subsubsection{Aggregation Scale}

Throughout this process, it was important to bear in mind the aggregation scales implemented. With the method developed in this project, the results of the space-time cube can vary greatly depending on how the data is aggregated both spatially and 
temporally. For instance, this project aggregated the data by block group and a threemonth interval. Depending on the spatial distribution of the data, some block-group time periods may only a small number of data points to calculate the mean from. Combined with how the data is temporally aggregated, either aggregated forward from the first transaction date in the dataset or aggregated backward from the last transaction date in the dataset, some transaction may be aggregated into a different space-time bin. This would thereby result in different spatio-temporal trends throughout the data.

\subsection{Machine Learning Results}

The results of the machine learning portion of the project consisted of statistical measurements of the prediction outputs. The measurements consisted of the average Mean Absolute Error (MAE), Root Mean Square Error (RMSE), and R-squared (R2) of the 10 iterations of the Multiple Linear Regression (MLR) and Random Forest Regression (RFR) algorithms. The results of both can be seen in Table 3.

Table 3. Average results of MLR and RFR models.

\begin{tabular}{|l|c|c|c|}
\hline \multicolumn{1}{|c|}{ Model } & AVG MAE & AVG RMSE & AVG R2 \\
\hline $\begin{array}{l}\text { Multiple Linear } \\
\text { Regression }\end{array}$ & $\$ 46,633.63$ & $\$ 74,459.59$ & 0.782 \\
\hline $\begin{array}{l}\text { Random Forest } \\
\text { Regression }\end{array}$ & $\$ 48,499.03$ & $\$ 76,671.25$ & 0.766 \\
\hline
\end{tabular}

As can be seen in Table 3, the MLR model with backward elimination performed better than the RFR model. Both the average MAE and RMSE for the MLR model were smaller than the average results of the RFR model. Furthermore, the average R2 of the MLR model was higher than the R2 of the RFR model. Taken together, the error between the predicted outcomes and the actual values of the MLR were smaller than the errors of the RFR model, and the variance in home values was better explained by the independent assessor features in the MLR model.

However, despite the MLR model performing better than the RFR model, both models present an unsatisfactory prediction ability. This is no doubt a result of the limited nature of the assessor data used in the models. Summary statistics of the dataset used can be seen in Table 4. Some variables of note include the lot size, square footage, year built, and value transfer. As can be seen in Table 4, lot size has a very large standard deviation of 57,924.03 square feet and a large range of 2,153,760 square feet. Similarly large ranges exist in the square footage, year built, and value transfer columns. These large disparities exist even after removing values smaller than minus three standard deviations from the mean. As the year built is a categorical considered a categorical variable, the mean and standard deviation descriptive statistics were not applicable. When compared to the summary statistics of the entire assessor dataset, as seen in Appendix D., the sample dataset taken is similar and representative of the entire dataset. The largest differences that exist between the two are a larger max lot size and square footage in the population dataset. However, despite these larger maximums, the median values of the lot size and square footage are very similar between the two datasets, and as such, the maximums of the population dataset do not present an important skewing effect. 
As mentioned in Section 5.2.2, the MLR model implemented backward elimination to remove any variables that were not of a significance level of 0.05 . Upon examination of the results of the backward elimination process, all variables present in the dataset were found to be significant. As such, given the R2 of 0.769 , the assessor data used in this project is no doubt useful for the prediction of home values. However, due to the large MAE and RMSE, it is apparent that more information is necessary for accurate predictions of home values.

Table 4. Summary statistics of sample dataset variables.

\begin{tabular}{|l|r|r|r|r|r|r|r|}
\hline Index & \multicolumn{1}{|l|}{ Mean } & Std. Dev. & \multicolumn{1}{l|}{ Min } & Q1 & Median & Q3 & Max \\
\hline Lot Size & 19,241 & 57,924 & 2,460 & 7,049 & 8,150 & 14,542 & $2,156,220$ \\
\hline $\begin{array}{l}\text { \# } \\
\text { Bathrooms }\end{array}$ & 2.17 & 0.685 & 0.25 & 2 & 2 & 2.5 & 6 \\
\hline $\begin{array}{l}\text { \# } \\
\text { Bedrooms }\end{array}$ & 3.32 & .85 & 1 & 3 & 3 & 4 & 8 \\
\hline \# Rooms & 6.75 & 1.75 & 2 & 5 & 6 & 8 & 11 \\
\hline \# Stories & 1.328 & .477 & 1 & 1 & 1 & 2 & 3 \\
\hline Sq. Ft. & $1,810.53$ & 706.39 & 572 & 1,300 & 1,651 & $2,207.5$ & 6,854 \\
\hline Year Built & $\mathrm{n} / \mathrm{a}$ & $\mathrm{n} / \mathrm{a}$ & 1801 & 1964 & 1986 & 2000 & 2014 \\
\hline $\begin{array}{l}\text { Value } \\
\text { Transfer }\end{array}$ & 249,189 & 158,857 & 11,500 & 134,806 & 215,000 & 330,000 & $1,220,000$ \\
\hline $\begin{array}{l}\text { Median } \\
\text { Transfer }\end{array}$ & 218,567 & 71,317 & 139,000 & 160,000 & 204,000 & 255,000 & 395,000 \\
\hline
\end{tabular}

There are many variables relevant to a home's value, and the assessed features used represent only a small portion of them. For instance, social statistics of the surrounding geographic area, like that of crime rates or educational rankings, are important in the determination of a home's value. While the ZIP code variable used in this project applies some geographic influence over the results, it is too broad of a categorization to provide meaningful representation of these social factors. Due to these shortcomings, the models produced in this project do not present a viable method of prediction. Furthermore, as can be seen by the average error and $\mathrm{R} 2$ results, the prospect of creating a machine learning algorithm that can accurately predict home values from assessor is dubious, and larger datasets with more independent variables are needed.

\subsection{Summary}

This project developed and implemented a methodology of creating a space-time cube from historical home transaction data. To test the method's implementation, two spacetime cubes and corresponding analyses were created, one of the areas around Redlands, $\mathrm{CA}$ and one of the areas around Chino, CA. The method produced six outputs, 3D visualizations of the space-time cube, 2D hot spot analyses, and 3D hot spot analyses. Each output allows the user to develop unique insights into the spatio-temporal trends of home values in the areas of study. 
The second component developed by this project, two machine learning models, Multiple Linear Regression and Random Forest Regression, to predict home values from assessor features, were also developed. The models were run on a sample dataset 10 times, and the results of each were summarized by average Mean Absolute Error (MAE), Root Mean Square Error (RMSE), and R-squared (R2). From these results it was determined that the models were not robust enough to accurately predict home values solely from assessor information, and that further independent variables, like those of crime rates and educational ranking, would need to be introduced to improve the models' accuracies. 



\section{Chapter 7 - Conclusions and Future Work}

This chapter provides a summary of the project and its conclusions, as well as suggests future work that can be done in the study areas covered by the project. Section 7.1 briefly discusses the processes involved with the development and competition of the project, as well as the conclusions reached by it. Section 7.2 discusses the shortcomings of the project, and the suggested work that can be done in the future to address these shortcomings.

\subsection{Conclusions}

The client of this project sought a method of visually conveying and statistically analyzing spatio-temporal trends in historical home transaction data. Previous methods implemented by the client were static, difficult to analyze statistically, and cumbersome to use for comparison between temporal intervals. To achieve these goals, this project developed a method of applying space-time cubes to the historical home transaction data.

Before development of the process could begin, the data provided by the client required a significant amount of cleaning and processing to prepare it for use. Once the data were prepared, a workflow was developed to translate the data into a form suitable for implementation of a space-time cube. This transformation involved aggregating the points by block group and time interval, calculating the mean transaction value of each aggregated block group/time interval, and generating random points representing the mean transaction value of each block group/time interval. This generation of the random points was performed by a custom Python script. From these points, the Create Spacetime cube by Aggregating Points tool was applied, and the resulting space-time cube was visualized in 3D and analyzed for emerging hot spots in both 2D and 3D.

Beyond the development of a space-time cube methodology, this project also created two space-time cube and hot spot analyses examples as proof of efficacy. One space-time cube mapped the spatio-temporal trends of the home values in the area around Redlands, $\mathrm{CA}$, and the other mapped the trend of home values in the areas around Chino, CA. From these space-time cubes and hot spot analyses, potential explanations of the spatiotemporal trends on display were developed.

Finally, a secondary aspect of this project involved the development of two machine learning models to predict home values from assessor information. Again, before implementation of the models, the assessor data required a significant amount of processing and cleaning for use in the models. Once the data were processed, two machine learning models, a Multiple Linear Regression model with backward elimination and a Random Forest Regression model, were chosen for implementation. A sample of the processed dataset was taken, and each model was applied to it ten times, with the average Mean Absolute Error, Root Mean Square Error, and R-squared calculated for each model. These measurements were then compared to determine which model was more effective. While the Multiple Linear Regression model performed the best of the two, the predictions made by it were still not particularly accurate. This is likely due to the limited information of the dataset missing key variables that influence a home's value, like that of a neighborhood's crime rate or educational ranking. 


\subsection{Future Work}

Regarding the space-time cube methodology, due to time constraints of this project and the difficulty in preparing the data for use, future work in this area would benefit from more thorough data preparation. In particular, due to the historical transaction dataset provided not containing information regarding the property use code, this project relied on a cutoff of $\$ 1,500,000$ to eliminate skewing from commercial property. In the future, implementations of the space-time cube method developed in this project should ensure any property not intended for single family residence is removed from the dataset.

Regarding the machine learning portion of this project, as mentioned in the results and conclusion section of this paper, the models produced in this project were not robust or accurate in their predictions. For future work in this area, more extensive research into the implementation of machine learning algorithms should be performed, and more importantly to the prediction of home values, a larger and more comprehensive variety of data should be applied. In particular, geographic social statistics, like those of neighborhood crime rate and educational ranking, are likely important variables in the prediction of a home's value. Furthermore, utilizing the entirety of the dataset, or at least a larger sample of the dataset, may allow for more accurate predictions. Finally, a further extension of the machine learning portion of this project would be to implement the prediction results derived from the models spatially. By doing this, future research could determine if there are any spatial patterns in the accuracy of predictions from the model, as well as present a visual representation of the predictions. 


\section{Works Cited}

Baldominos, A., Blanco, I., Moreno, A., Iturrarte, R., Bernárdez, Ó., \& Afonso, C. (2018). Identifying Real Estate Opportunities Using Machine Learning. Applied Sciences.

Esri a. (2019). Create Space Time Cube. Retrieved from arcgis.com: http://desktop.arcgis.com/en/arcmap/10.3/tools/space-time-pattern-miningtoolbox/create-space-time-cube.htm

Esri b. (2019). Visualizing the Space Time Cube. Retrieved from arcgis.com: http://desktop.arcgis.com/en/arcmap/10.3/tools/space-time-pattern-miningtoolbox/visualizing-cube-data.htm\#

Esri c. (2019). How Emerging Hot Spot Analysis works. Retrieved from ArcGIS Desktop: http://desktop.arcgis.com/en/arcmap/latest/tools/space-time-pattern-miningtoolbox/learnmoreemerging.htm

Getis, A., \& Ord, J. (1992). The Analysis of Spatial Association by Use of Distance Statistics. Geographical Analysis, 189-206.

Goodchild, M. F. (2013). Prospects for a Space-Time GIS. Annals of the Association of American Geographers, 1072-1077.

Hagerstrand, T. (1970). What about people in Regional Science? Papers of the Regional Science Association, 24(1), 6-21.

Kaboudan, M., \& Sarkar, A. (2007). Forecasting prices of single family homes using GIS-defined neighborhoods. Journal of Geographical Systems, 23-45.

Kang, Y., Cho, N., \& Son, S. (2018). Spatiotemporal characteristics of elderly population's traffic accidents in Seoul using space-time cube and space-time kernel density estimation. PLOS One, 13(5), 1-17.

Kraak, M. -J. (2003). Geovisualization and Time- New Opportunities for the SpaceTime Cube. Proceedings of the 21 st International Cartographic Conference (ICC), 293-306.

Kuhn, M., Schularick, M., \& Steins, U. (2018, August 9). Asset prices and wealth inequality. Retrieved from Voxeu.org: https://voxeu.org/article/asset-prices-andwealth-inequality

Kwan, M.-P. (2000). Interactive geovisualization of activity-travel patterns using threedimensional geographical information systems: a methodological exploration with a large data set. Transportation Research Part C: Emerging Technologies, 8(1-6), 185-203.

Kwan, M.-P. (2008). Gender, the Home-Work Link, and Space-Time Patterns of Nonemployment Activities*. Economic Geography, 75(4), 370-394.

Malpezzi, S. (2017). Residential real estate in the US financial crisis, the Great Recession, and their aftermath. Jing Ji Lun Wen Cong Kan, 5-56.

McClendon, L., \& Meghanathan, N. (2015). Using Machine Learning Algorithms to Analyze Crime Data. Machine Learning and Applications: An International Journal, 1-12.

Median Home Value Southern California. (2019). Retrieved from laalmanac.com: http://www.laalmanac.com/economy/ec37.php

Moenius, J. (2011, December 20). Inland Empire Housing Bubble. Retrieved from youtube.com: https://www.youtube.com/watch?v=SWfhqeoszho 
Nakaya, T., \& Yano, K. (2010). Visualising Crime Clusters in a Space-time Cube: An Exploratory Data-analysis Approach Using Space-time Kernel Density Estimation and Scan Statistics. Transactions in GIS, 14(3), 223-239.

Rosen, S. (1974). Hedonic Prices and Implicit Markets: Product Differentiation in Pure Competition. Journal of Political Economy, 34-55.

Southern California Association of Governments (SCAG). (2019). Profile of the City of Redlands. Los Angeles: Southern California Association of Governments (SCAG).

Stewart, M. (2019, March 24). The actual difference between statistics and machine learning. Retrieved from towardsdatascience.com: https://towardsdatascience.com/the-actual-difference-between-statistics-andmachine-learning-64b49f07ea3

Unidata. (2019). netCDF Introduction. Retrieved from unidata.ucar.edu: https://www.unidata.ucar.edu/software/netcdf/docs/netcdf_introduction.html 


\section{Appendix A. Scripts Written for Data Cleaning}

Code for joining and cleaning the assessor data text files:

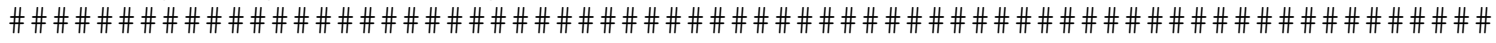
\#\#\#\#\#\#\#\#\#\#

\# Process and clean assessor data files

\# Author - Breck Polk

\# Purpose: Process and clean assessor data text files

\# Date - 11-28-2019

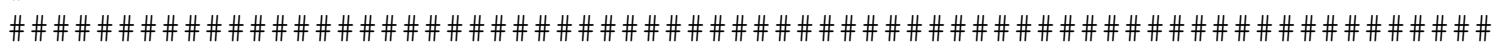
\#\#\#\#\#\#\#\#\#\#

\# Import Libraries

import re

import pandas as pd

\# Text files list

assessor_path_list $=[$

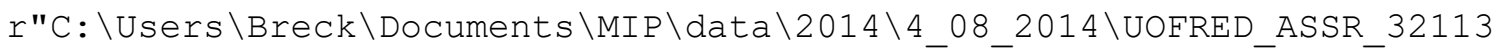
_DAT \UOFRED_ASSR_32113_DAT.txt",

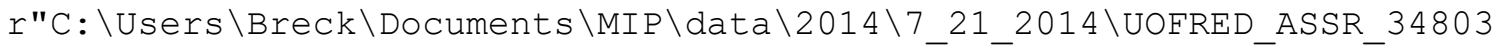
_DAT\UOFRED_ASSR_34803_DAT.txt",

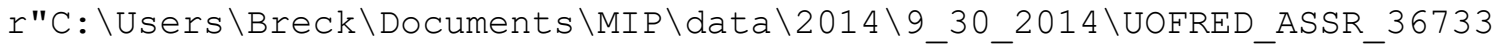
_DAT \UOFRED_ASSR_36733_DAT.txt",

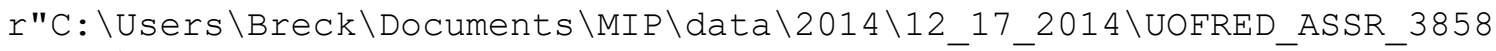

7_DAT \UOFRED_ASSR_38587_DAT.txt",

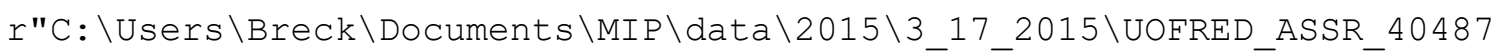
DAT\UOFRED_ASSR_40487_DAT.txt",

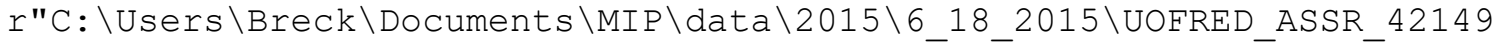
_DAT \UOFRED_ASSR_42149_DAT.txt",

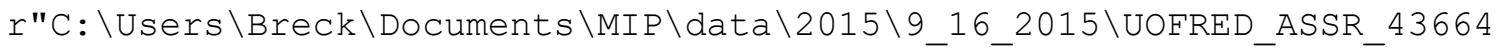
_DAT \UOFRED_ASSR_43664_DAT.txt",

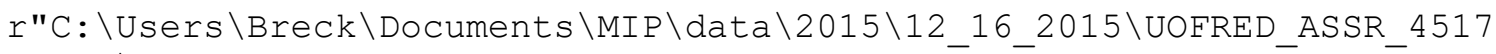
3_DAT\UOFRED_ASSR_45173_DAT.txt",

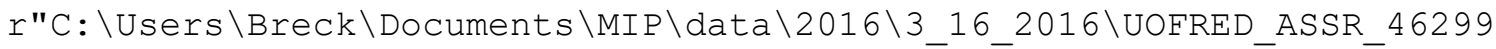
_DAT\UOFRED_ASSR_46299_DAT.txt",

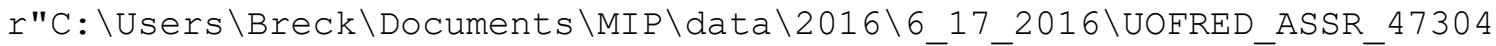
_DAT \UOFRED_ASSR_47304_DAT.txt",

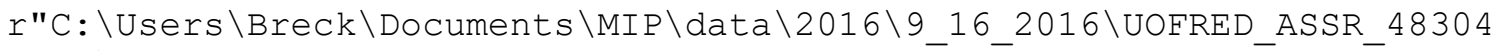
_DAT \UOFRED_ASSR_48304_DAT.txt", 


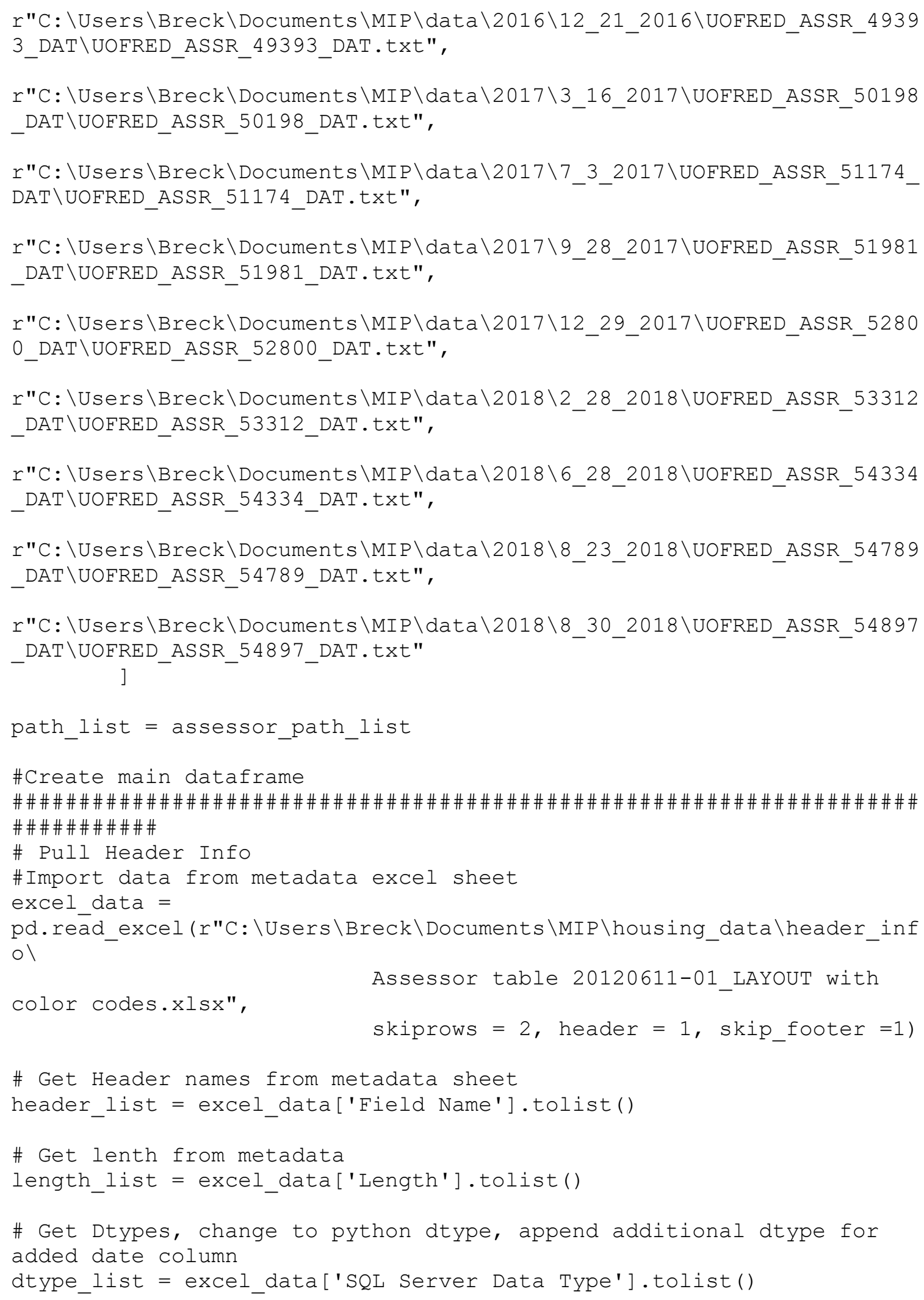




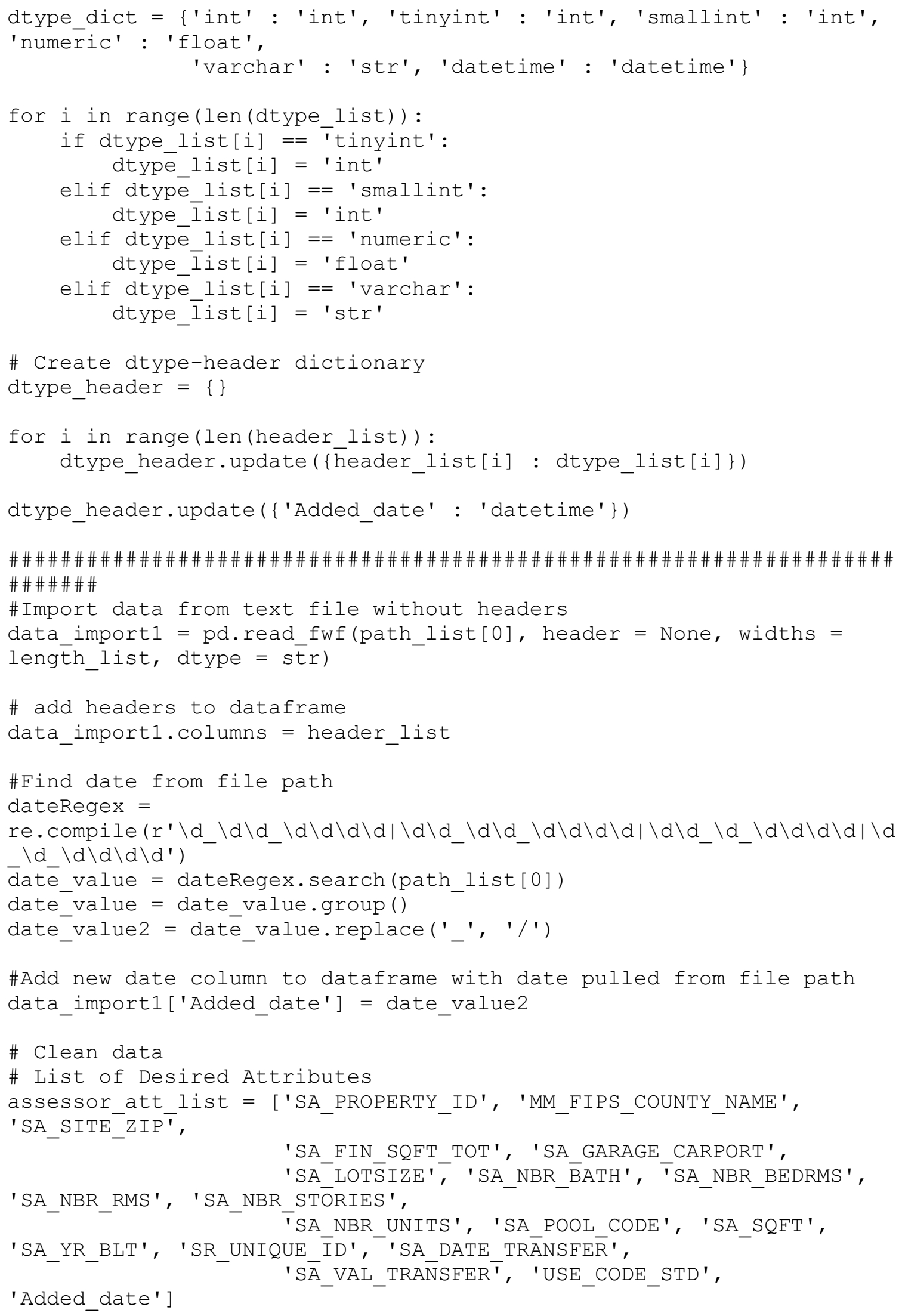




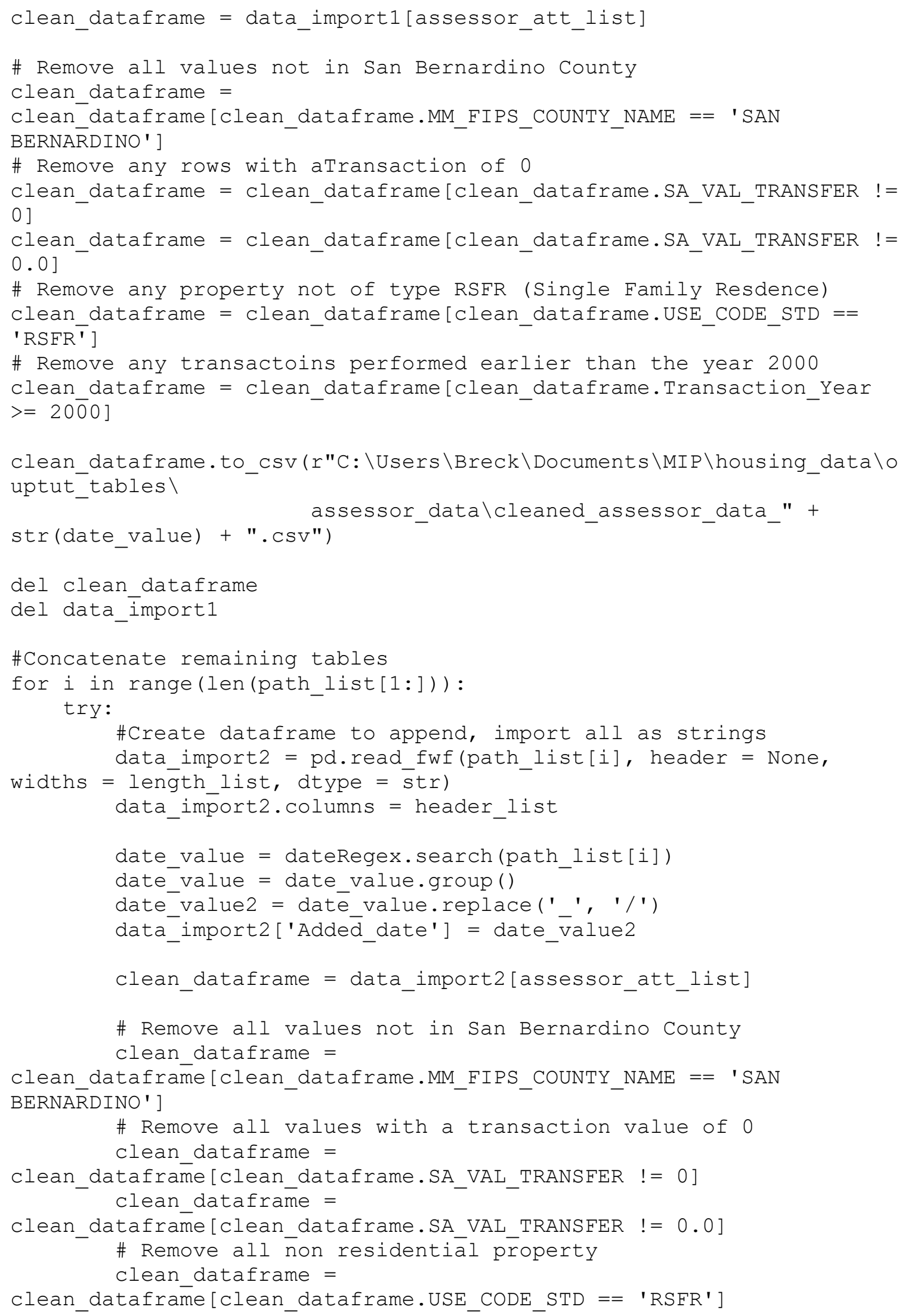


2000

\# Remove any transactoins performed earlier than the year

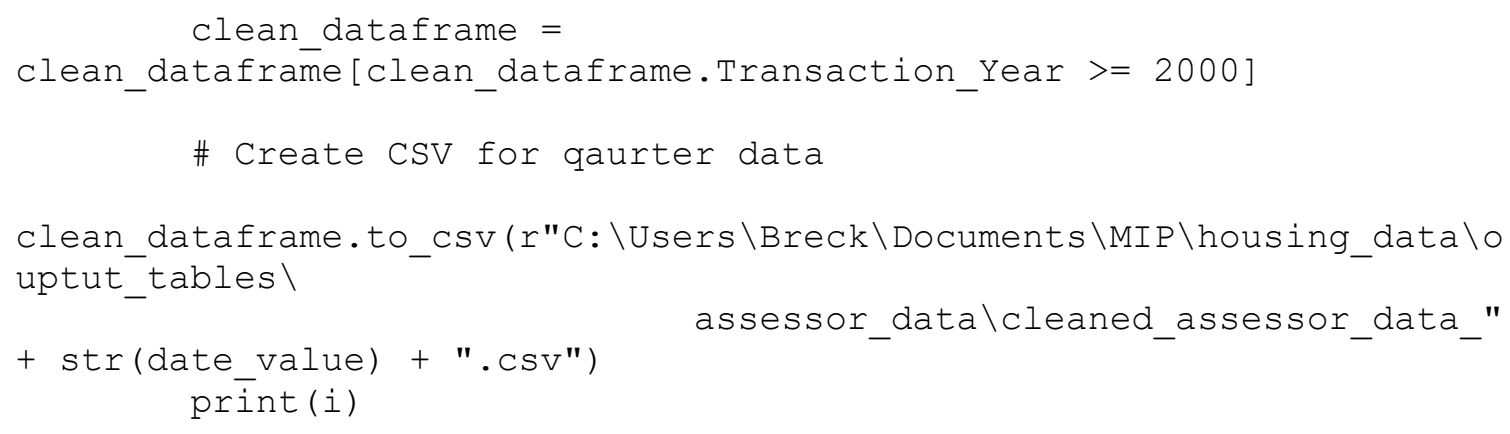




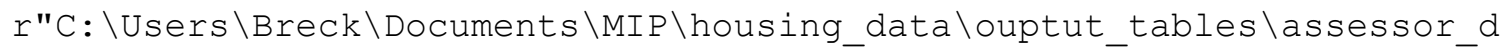
ata \cleaned_assessor_data_9_16_2016.csv",

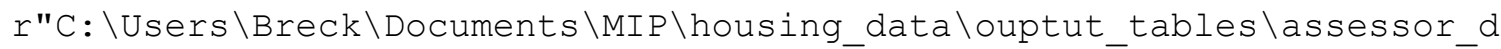
ata \cleaned_assessor_data_9_28_2017.csv",

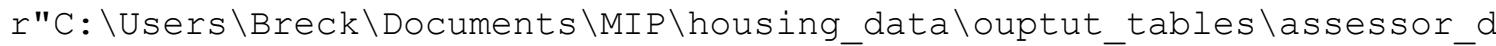
ata \cleaned_assessor_data_9_30_2014.csv",

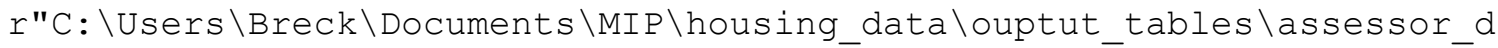
ata \cleaned_assessor_data_12_16_2015.csv",

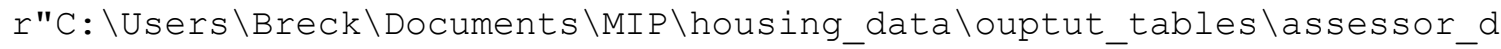
ata \cleaned_assessor_data_12_17_2014.Csv",

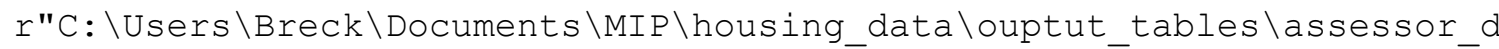
ata\cleaned_assessor_data_12_21_2016.csv",

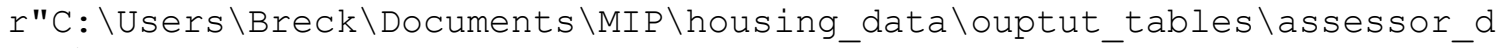
ata \cleaned_assessor_data_12_29_2017.csv"]

\# Create final table

final_table $=$ pd.read_csv(csv_list $[0]$, header $=0)$

for $i$ in range (len (csv_list $[1:]))$ :

data_import $1=$ pd.read_csv(csv_list[i], header $=0)$

final_table $=$ pd.concat ([final_table, data_importl],

ignore_index $=$ True)

\# Create column for last transaction year

def create_year (row) :

date $=\operatorname{str}($ row $[$ 'SA_DATE_TRANSFER' $])$

date $=$ date $[0: 4]$

return date

final_table['Transaction_Year'] = final_table.apply (lambda row:

create_year(row), axis=1)

\# Change NAN to $\mathrm{N}$ for pools

final_table['SA_POOL_CODE'].fillna('N', inplace = True)

\# Drop unnecessary columns

final_table.drop(['SA_VAL_MARKET', 'SA_VAL_FULL_CASH',

'USE_CODE_STD', 'USE_CODE_MUNI',

'Adde d_datē', 'SA_DATE_TRANSFER', 'SR_UNIQUE_ID',

'SA_APPRAISE_YR', 'SA_VAL_ASSD',

'ASSR YEAR',' 'SA SITE PLUS 4',

'SA PARCEL NBR PRIMA $\bar{R} Y$ ', 'Unnamed: 0.1 ', 'Unnamed: 0'], axis $=1$, inplace=Trüe)

\#Calculate and remove vlaues greater than 3 Std. Deviations from the mean

stddev = final_table.SA_VAL_STRANSFER.quantile $(0.997)$ 


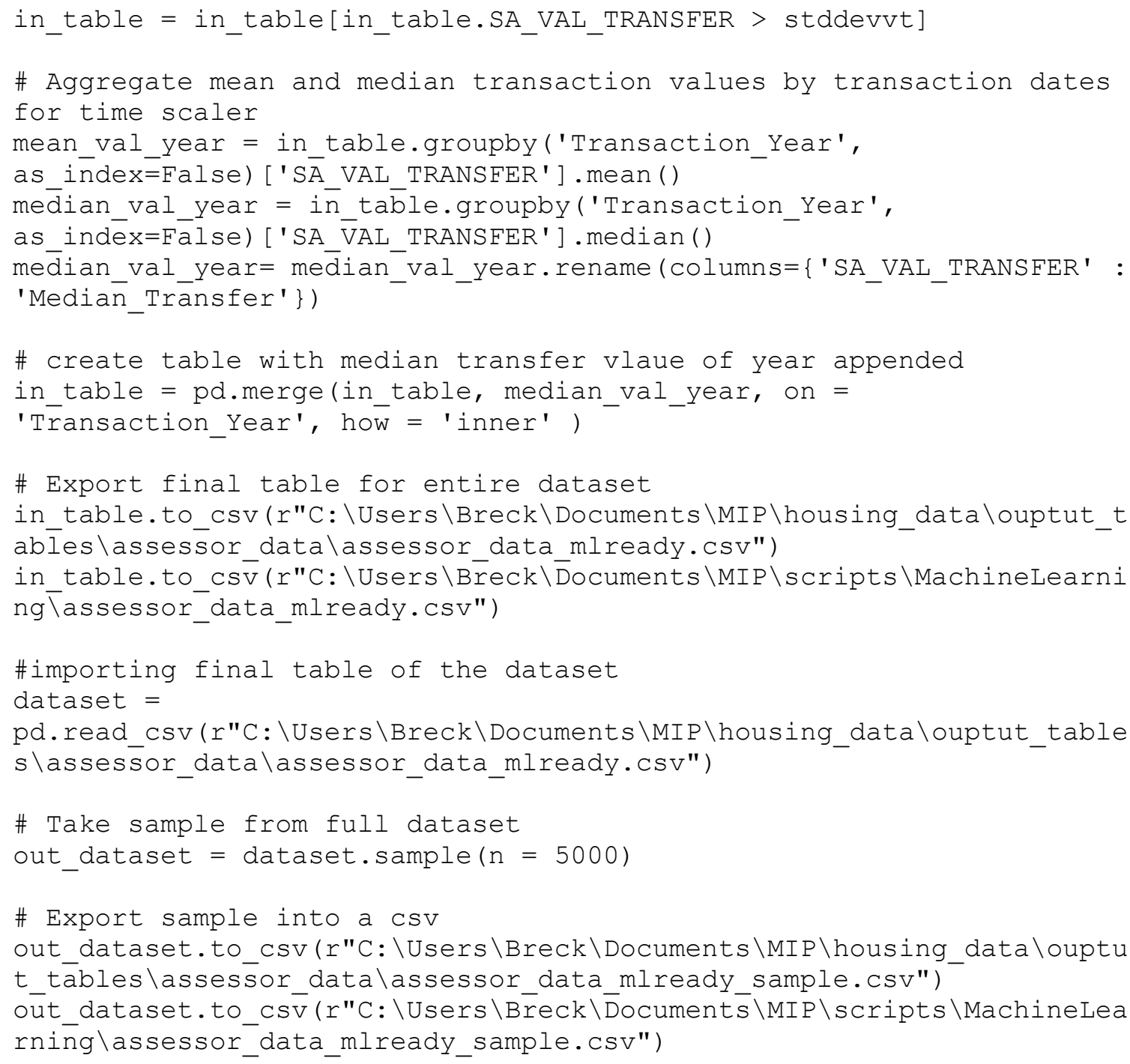

Code for joining and cleaning the historical transaction data text files:

\# Process and clean Historical Transaction Data

\# Author - Breck Polk

\# Purpose: Prepares Historical Transaction files for use with space-time cubes

\# Date - 11-28-2019

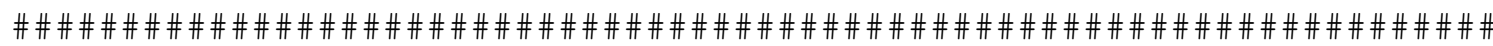
\#\#\#\#\# \#\#\# \#\#\#

\# Impot Libraries

import pandas as pd

import re

\# List of all historical transaction file paths history_path_list $=[$

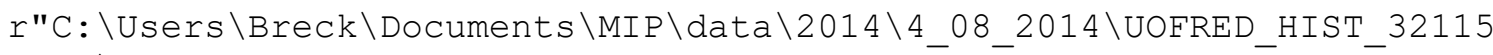
_DAT \UOFRED_HIST_32115_DAT.txt", 


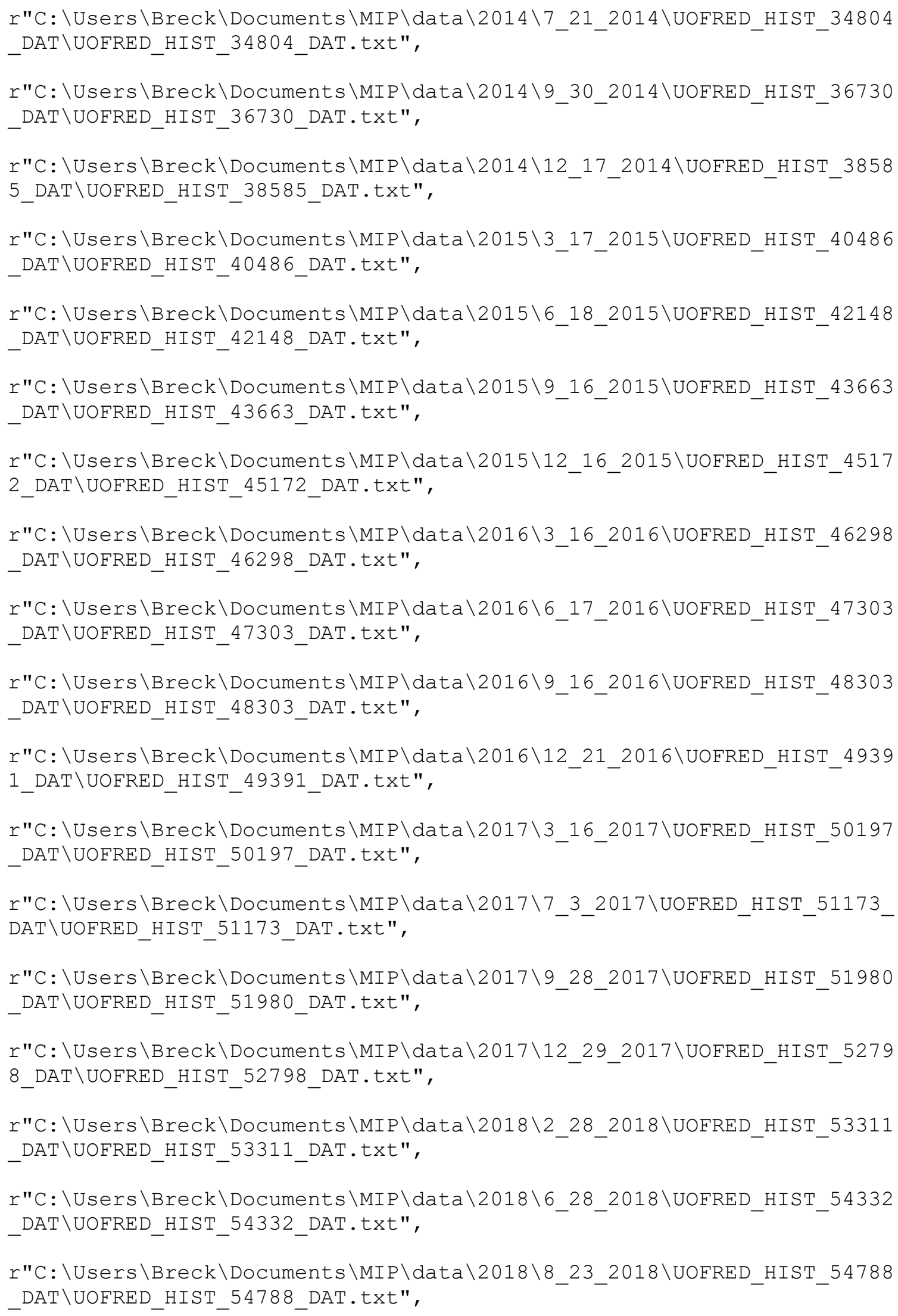




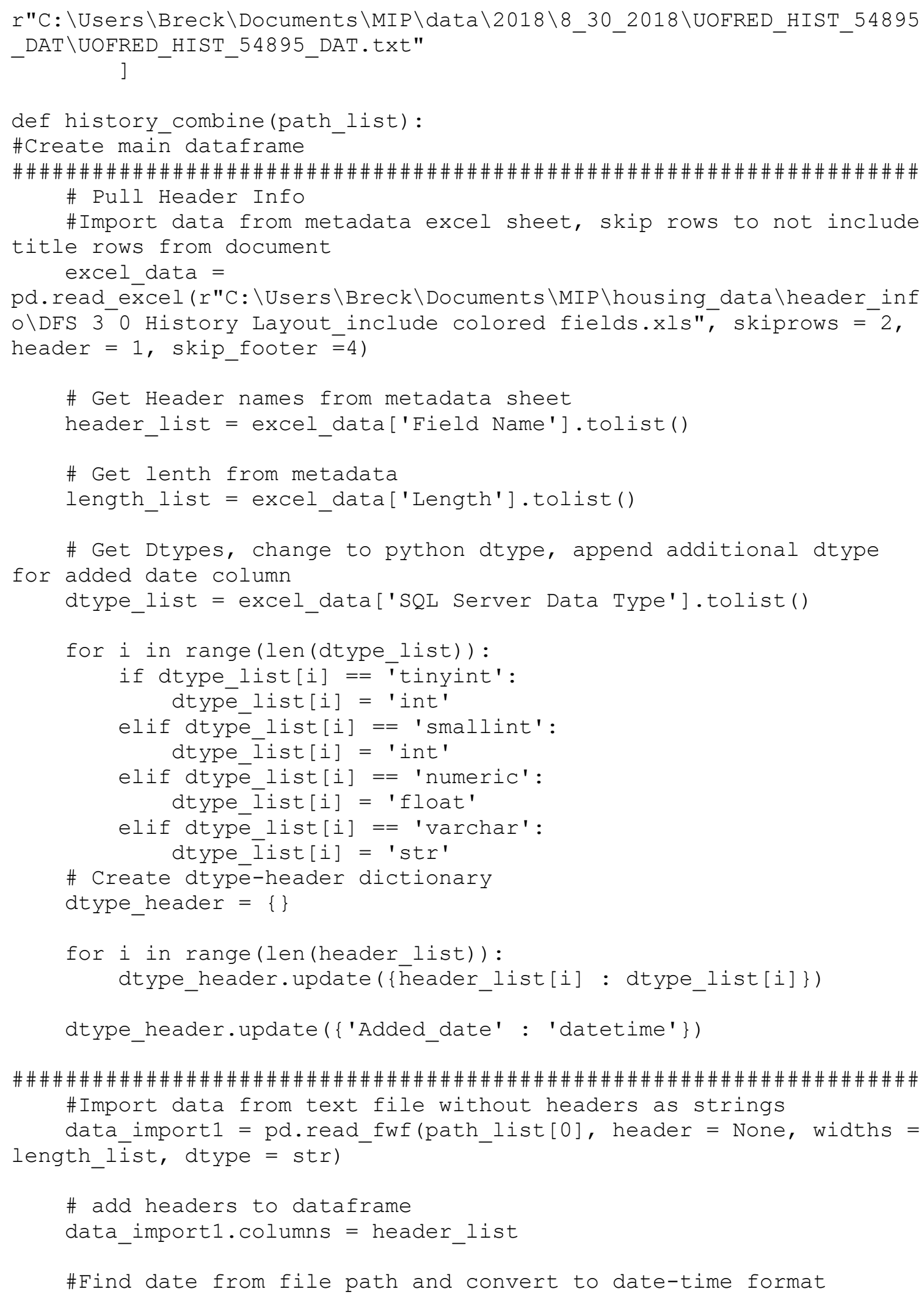




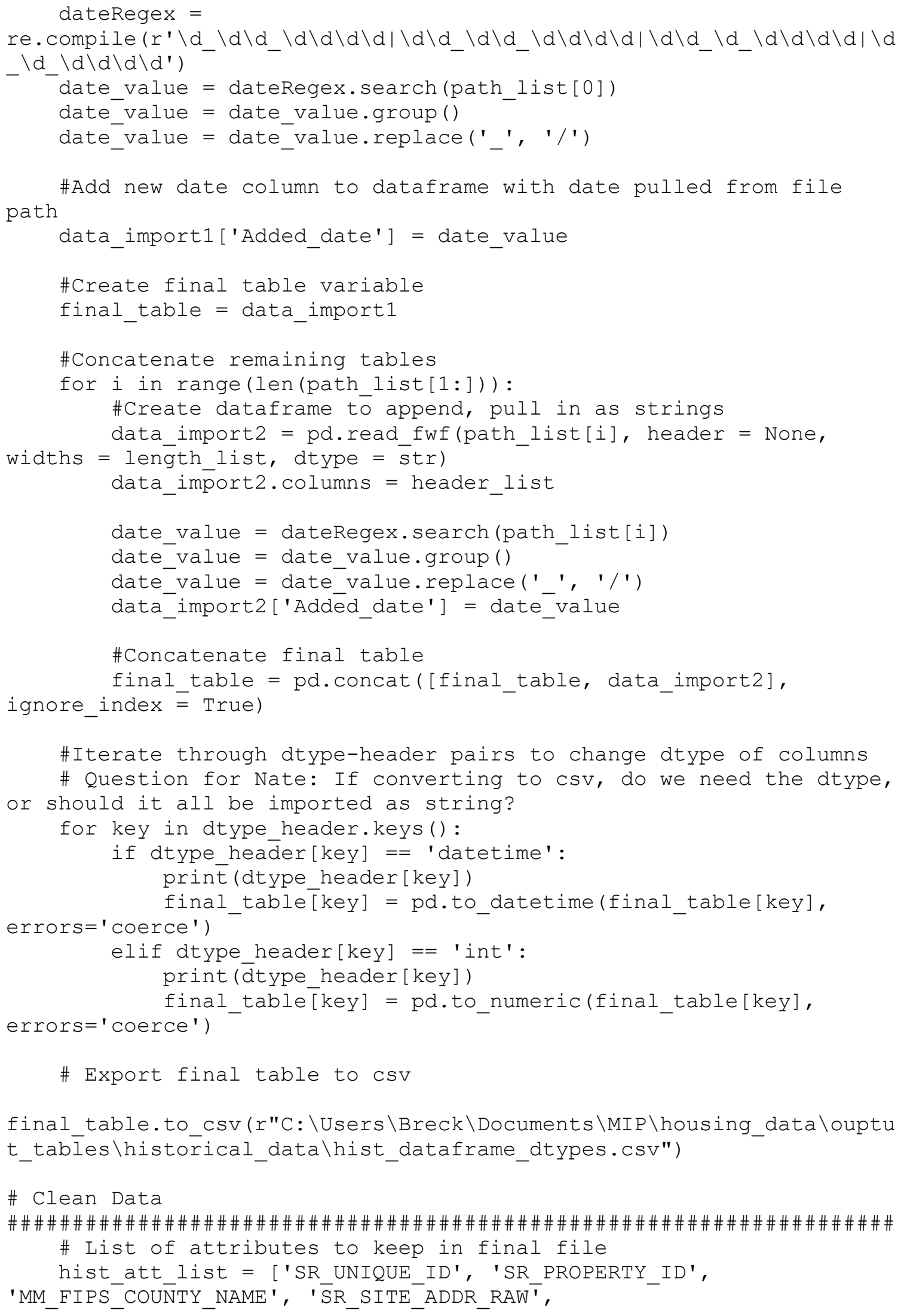




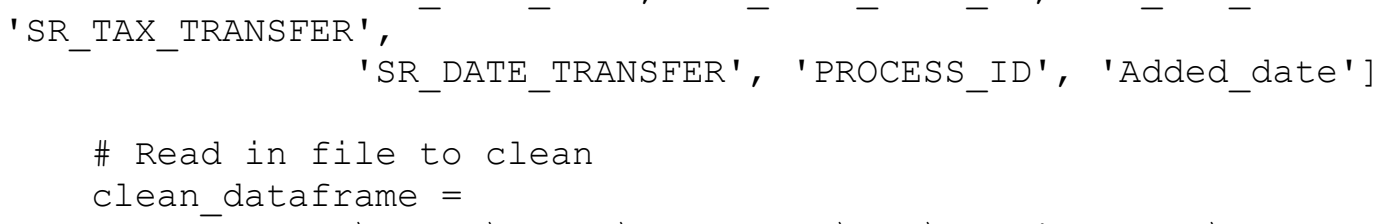

\section{Appendix B. Scripts written for Machine Learning}

Code for Multiple Linear Regression Model:

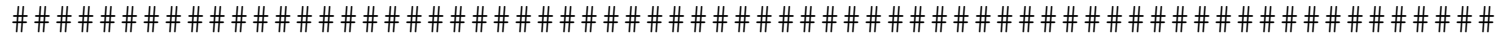

\# Multiple Linear Regression Test

\# Author - Breck Polk

\# Purpose: Test the MLR model multiple times to determine average errors and R2

\# Date - 12-11-2019 


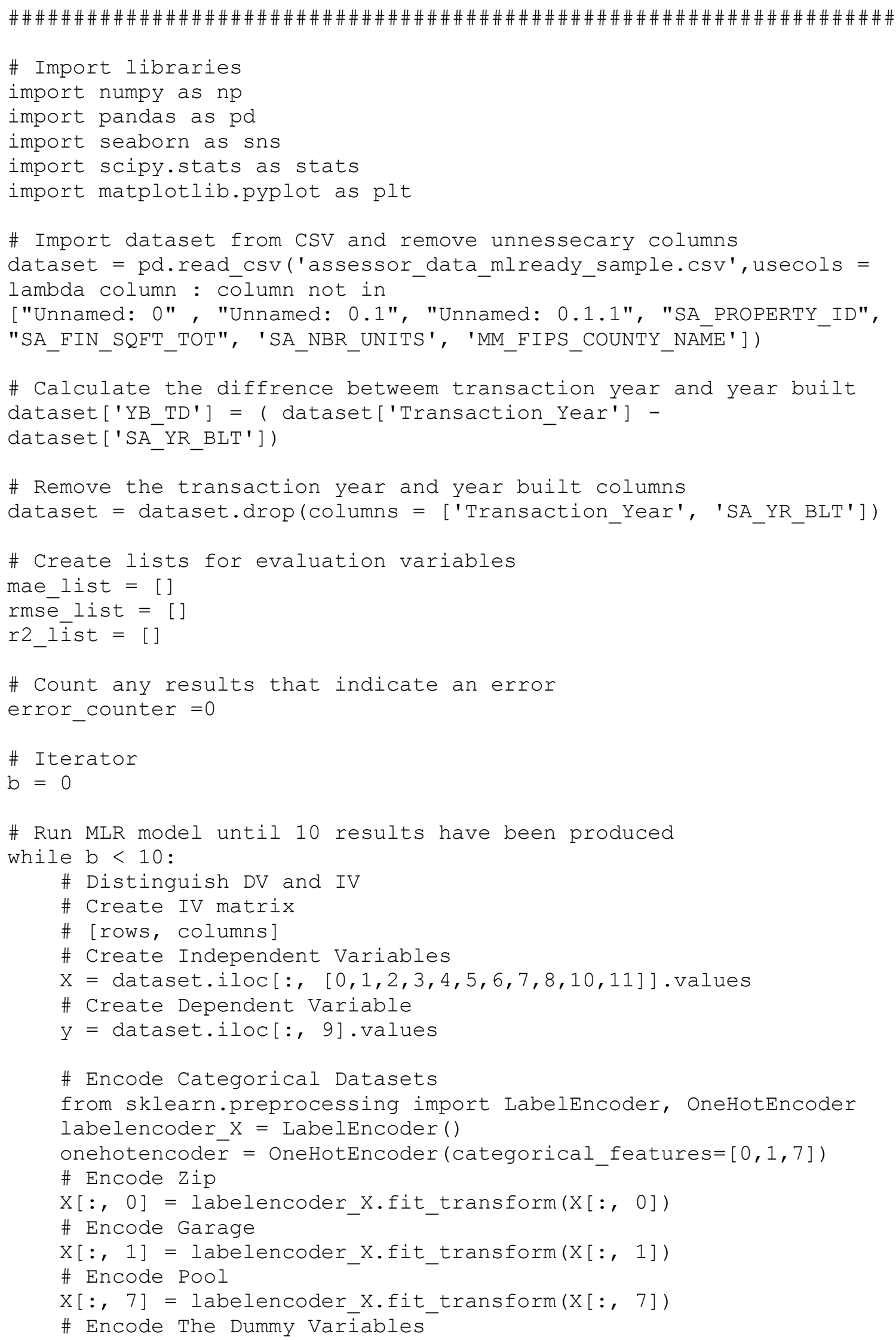




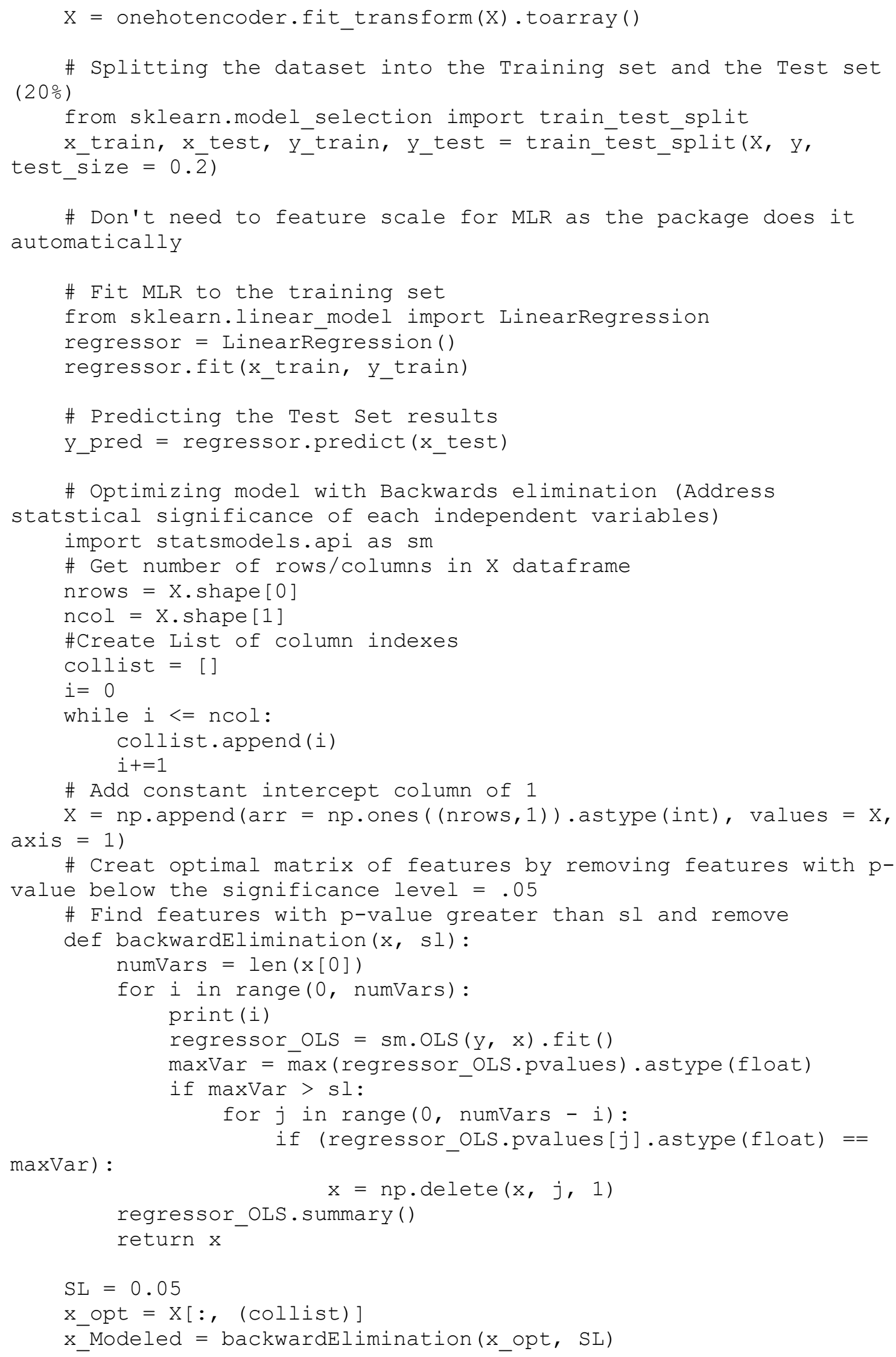




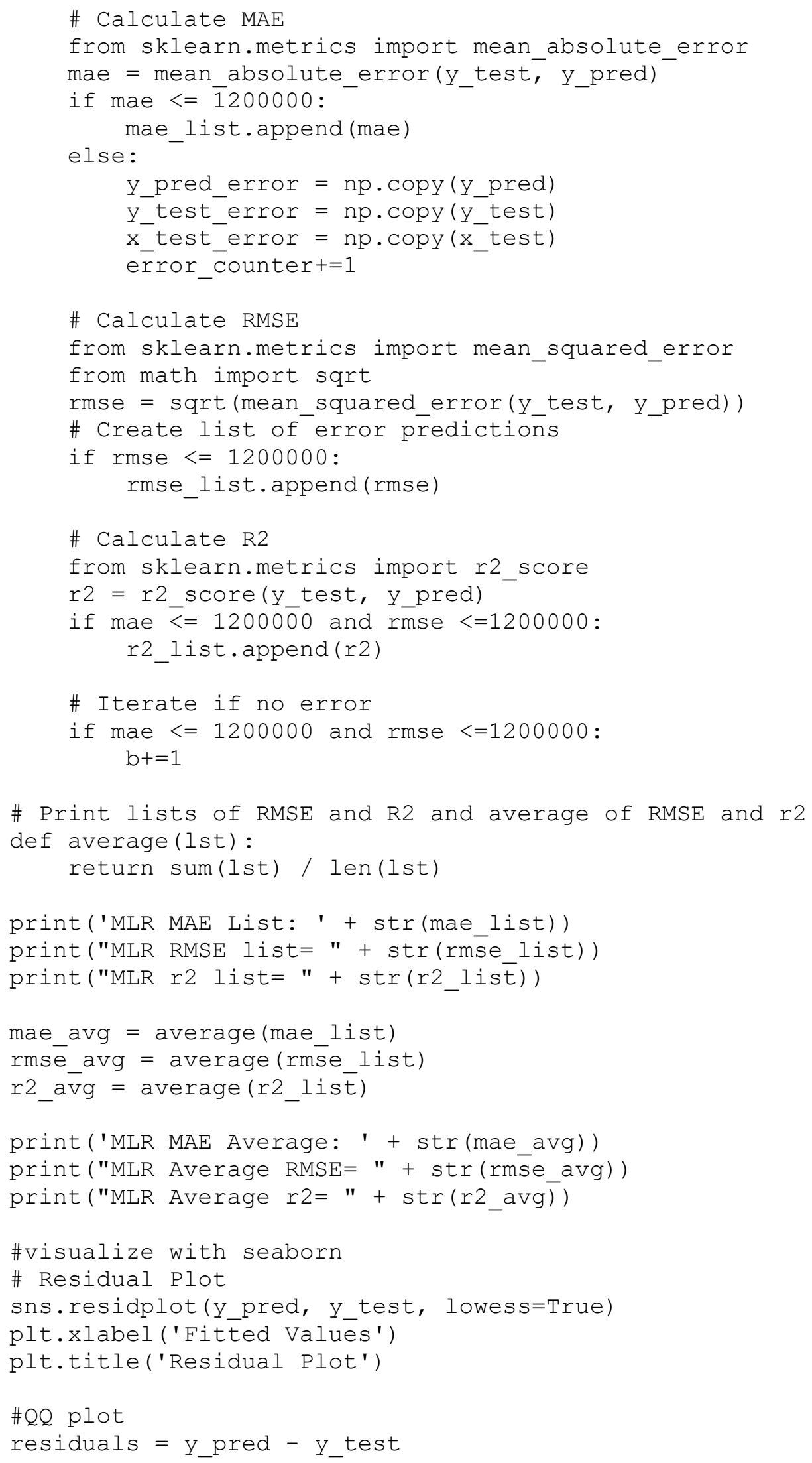


plt.figure (figsize $=(7,7)$ )

stats.probplot (residuals, dist="norm", plot=plt)

plt.title("Normal Q-Q Plot")

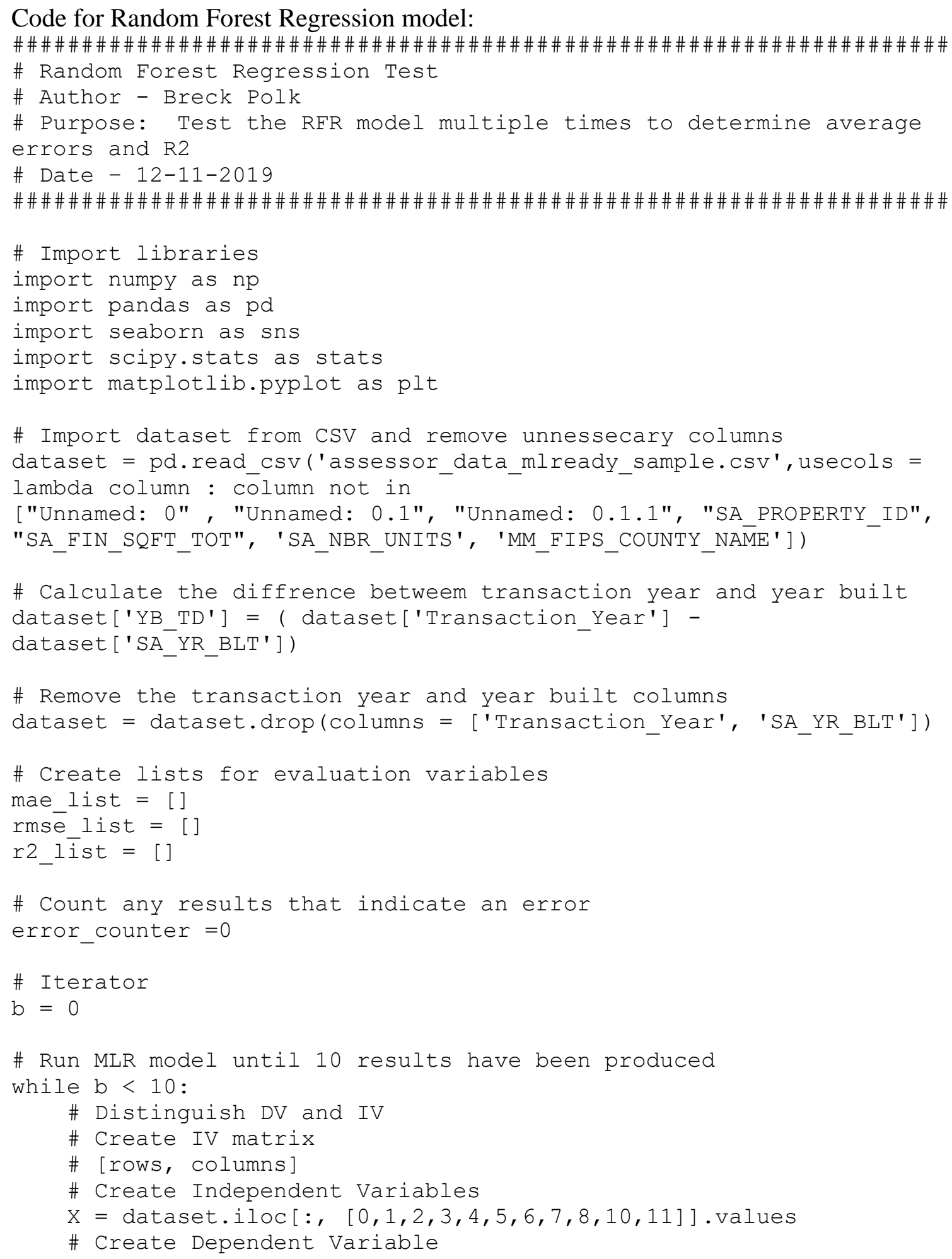




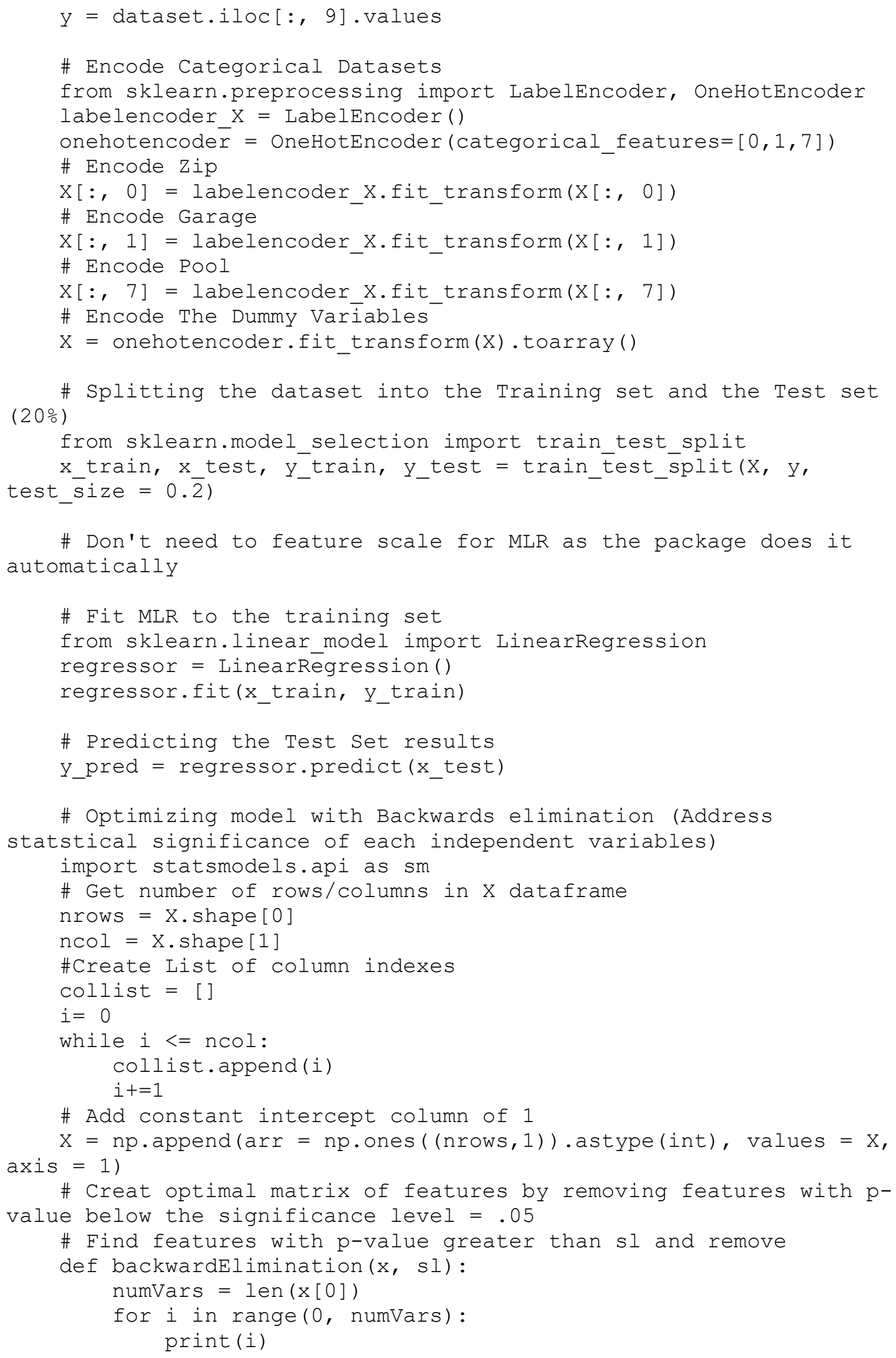




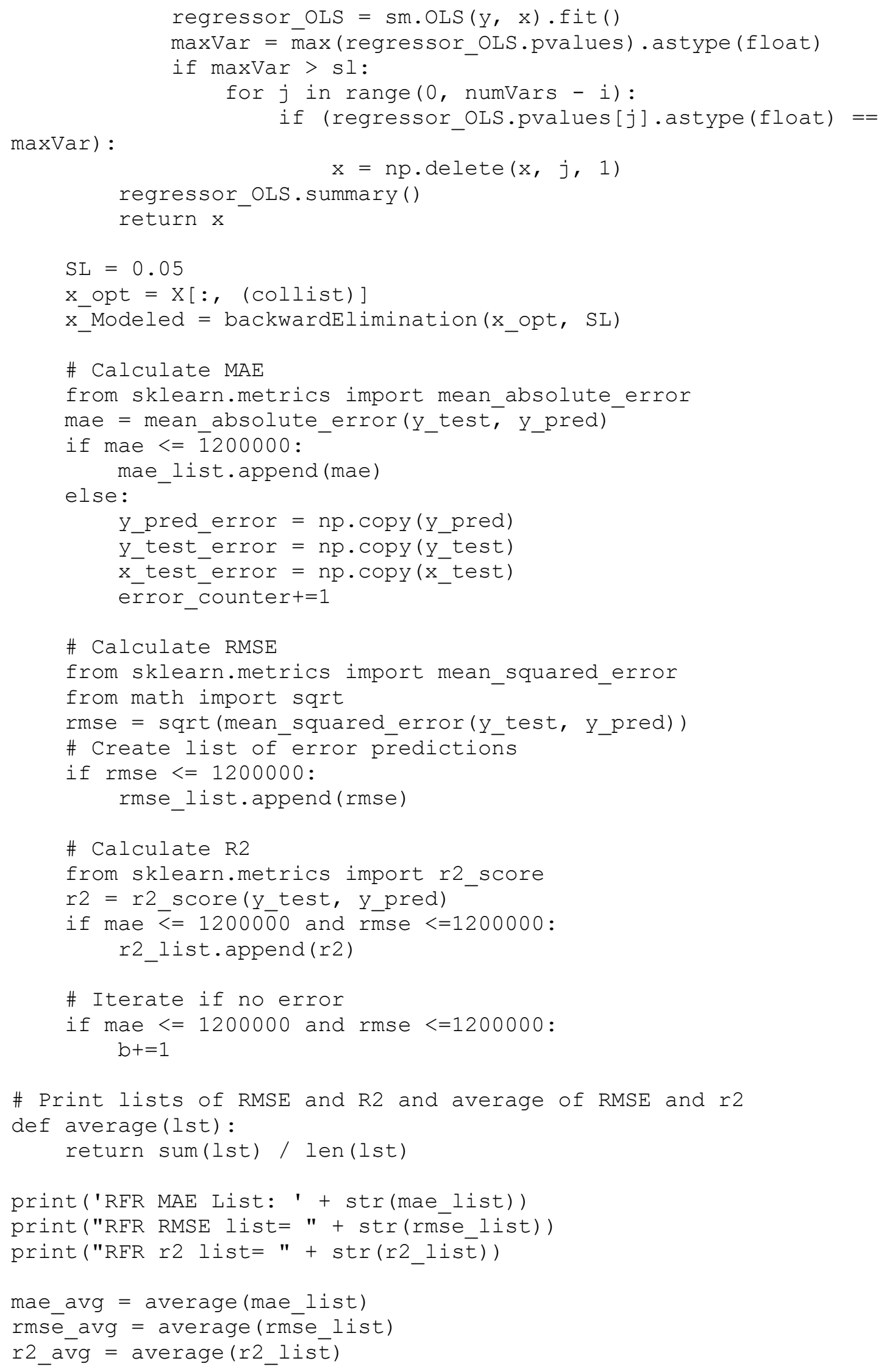




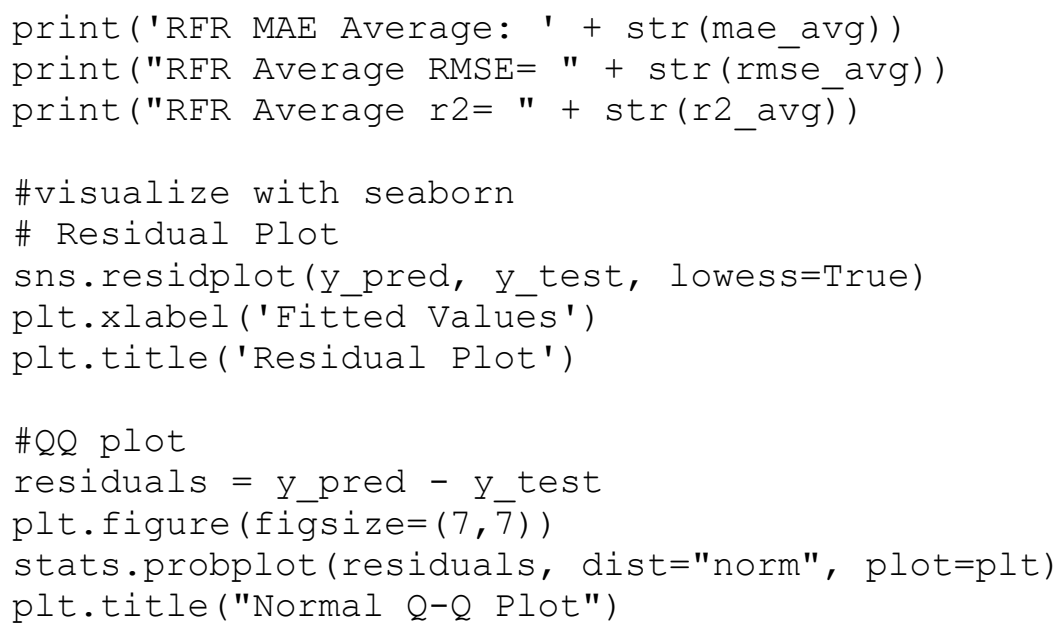

\section{Appendix C. Script for Random Point Generation}

Code for generating random points:

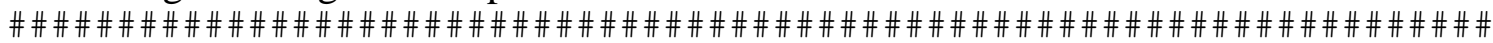

\# Generate random points of mean transaction value by block

group/quarter

\# Author - Breck Polk

\# Purpose: Generate random points of mean transaction value by

block group/quarter

\# Date - 11-28-2019

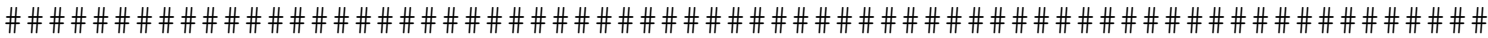

\# Import Libraies

import arcpy

\# Create workspace

arcpy.env. workspace $=$

"C:/Users/Breck/Documents/ArcGIS/Projects/stc_test/stc_test.gdb"

\# Establish geodatabase

gdb $=$

"C:/Users/Breck/Documents/ArcGIS/Projects/stc_test/stc_test.gdb"

arcpy.env.overwriteoutput = True

\# Feature class paths

\# Aggregate feautre

agg_points =

"C:/Users/Breck/Documents/ArcGIS/Projects/stc_test/stc_test.gdb/RSBL LHIGHMENT_Selected_AggregatePoints"

\# Block Group Polygon Feature

$\mathrm{blk}$ grps $=$

"C:/Users/Breck/Documents/ArcGIS/Projects/stc_test/stc_test.gdb/Red SB_LL_High_Ment_blks_Projected" 
\# Create Search cursors

agg_points_cursor = arcpy.da.SearchCursor(agg_points, ['OBJECTID',

'MEAN USER SR VAL TRANSFER', 'rand points', 'START DATE',

'poly id'])

agg_points_cursor.reset ()

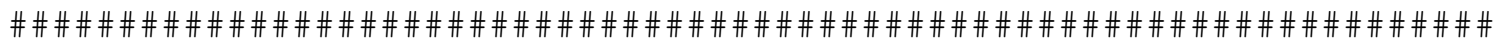

\# Create Random Points

expression = 'rand (!MEAN_USER_SR_VAL_TRANSFER!)'

codeblock = "" "

def rand (input):

points = input $/ 10000$

return points

" " "

\#Add field for random points

arcpy.AddField management (in_table=agg_points,

field_name='rañd_points', fiēld_type=' $\bar{S} H O R T$ ')

\#Add random points

arcpy.CalculateField_management(agg_points, 'rand_points',

expression, "PYTHON3", codeblock)

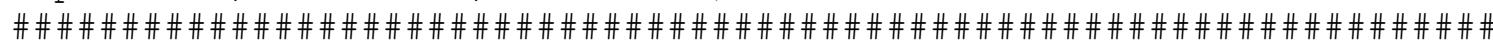

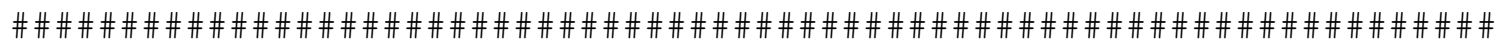

\# Create Date String

expression1 = 'string (!START DATE!)'

codeblock1 = "" "

def string (input):

list = input.split(' ')

date $=$ list $[0]$

return date

" " "

\#Add field for date string

arcpy.AddField_management (in_table=agg_points,

field_name='date_string', fiēld_type='TEXT')

\#Add $\bar{d}$ ate

arcpy.Calculatefield management (agg points, 'date string',

expression1, "PYTHON$\overline{3} "$, codeblock1)

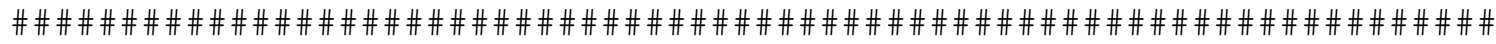

\# Create final random point table from template

final_table_template $=$

"C:/Ūsers/Breck/Documents/ArcGIS/Projects/stc_test/stc_test.gdb/fina l_table_template"

a $\bar{r}$ cpy.CréreateFeatureclass_management (gdb, "final_table", "PoINT", final table template)

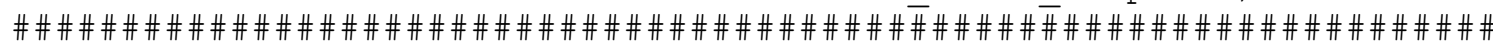

\# Loop counter

$\mathrm{n}=0$

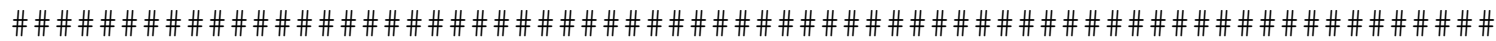

\#Create Random Points

for row in agg_points_cursor:

\#Variables for fearures 


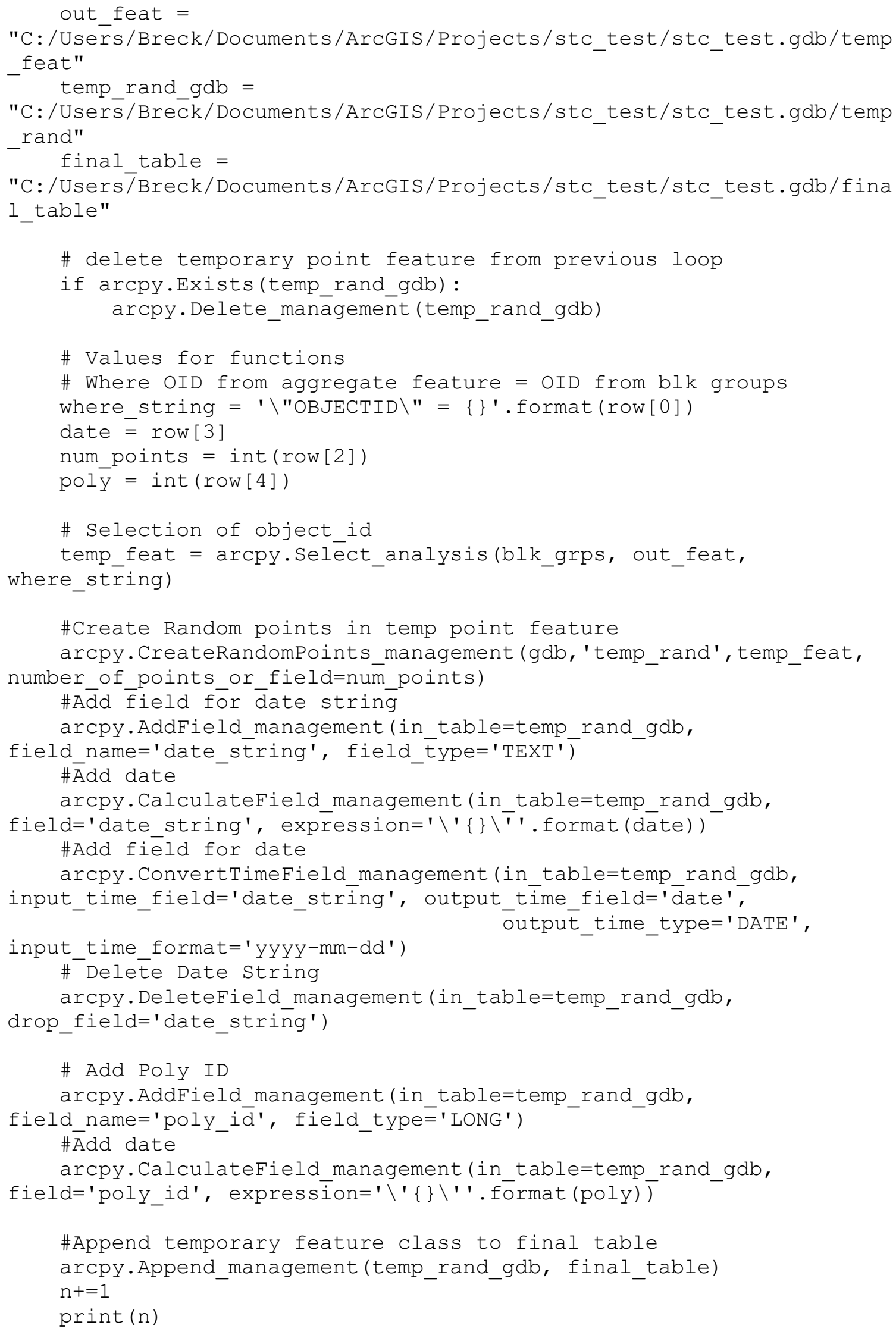




\section{Appendix D. Summary Statistics for Entire Assessor Dataset}

\begin{tabular}{|l|r|r|r|r|r|r|r|}
\hline Index & \multicolumn{1}{|l|}{ Mean } & Std. Dev. & \multicolumn{1}{l|}{ Min } & Q1 & Median & \multicolumn{1}{l|}{ Q3 } & \multicolumn{1}{l|}{ Max } \\
\hline Lot Size & 18,385 & 65,154 & 2,401 & 7,040 & 8,100 & 13,960 & $8,376,588$ \\
\hline $\begin{array}{l}\text { \# Bathrooms } \\
\text { \# }\end{array}$ & 2.16 & 0.68 & 0.25 & 2 & 2 & 2.5 & 10 \\
Bedrooms & 3.31 & 0.858 & 1 & 3 & 3 & 4 & 11 \\
\hline \# Rooms & 6.73 & 1.74 & 1 & 5 & 7 & 8 & 11 \\
\hline \# Stories & 1.323 & 0.472 & 1 & 1 & 1 & 2 & 3 \\
\hline Sq. Ft. & $1,799.53$ & 705.11 & 553 & 1,288 & 1,650 & 2,179 & 17,468 \\
\hline Year Built & $\mathrm{n} / \mathrm{a}$ & $\mathrm{n} / \mathrm{a}$ & 1801 & 1964 & 1986 & 2000 & 2017 \\
\hline $\begin{array}{l}\text { Value } \\
\text { Transfer }\end{array}$ & 247,563 & 158,781 & 10,091 & 134,500 & 210,000 & 325,000 & $1,249,000$ \\
\hline $\begin{array}{l}\text { Median } \\
\text { Transfer }\end{array}$ & 217,738 & 70,262 & 139,000 & 160,000 & 204,000 & 255,000 & 395,000 \\
\hline
\end{tabular}

